\title{
EACPT Virtual Meeting 2021
}

Focus on Progress in Clinical Pharmacology

28-29 June 2021

\section{The Abstracts}




\section{Poster Session 1a - PK-PD-DDI}

15

Population pharmacokinetics of oxycodone and active metabolites in patients with cancer-related pain

Mr. Bram Agema ${ }^{1,2}$, dr. Astrid Oosten ${ }^{1}$, dr. Sebastiaan Sassen², prof. dr. Carin Van der Rijt ${ }^{1}$, dr. Birgit Koch ${ }^{2}$, prof. dr. Ron Mathijssen ${ }^{1}$, dr. Stijn Koolen ${ }^{1,2}$

${ }^{1}$ Dept. of Medical Oncology, Erasmus MC Cancer Institute, Erasmus University Medical Center, Rotterdam, The Netherlands

${ }^{2}$ Dept. of Clinical Pharmacy, Erasmus University Medical Center, Rotterdam, The Netherlands

Background: Cancer-related pain is common and oxycodone is frequently used as treatment in this setting. It is largely unknown which factors affect the outcomes of treatment. Unraveling these factors may be a starting point for precision dosing of oxycodone in this population. Objectives: Our first aim was to develop a model to assess the pharmacokinetics (PK) of oxycodone and its metabolites and the influence of covariates in patients with cancer-related pain. The second aim was to assess the correlation between oxycodone (metabolite) area under the curve (AUC), pain control and adverse events.

Methods: Patients with cancer-related pain hospitalized at the Erasmus Medical Center between 2010 and 2014, who were titrated with extended (ER) and/or immediate release (IR) oral oxycodone participated. PK samples of oxycodone, oxymorphone, nor-oxycodone and nor-oxymorphone were taken every $12 \mathrm{~h}$ and occasionally at $5,15,30$ and $60 \mathrm{~min}$ after oxycodone IR administration. Patient-reported pain scores and the occurrence and severity of adverse events were collected every $12 \mathrm{~h}$. PK data was analyzed using NONMEM® v7.4. PK/pharmacodynamic (PK/PD) associations were tested using Pearson's correlation coefficient.

Results: In total, 1235 samples for oxycodone and its metabolites were collected in 28 patients. A five-compartment model best described oxycodone, nor-oxycodone and nor-oxymorphone pharmacokinetics. Oxycodone absorption was described by 2 separate first-order processes for oxycodone ER and IR tablets. As 58 percent of oxymorphone samples, also an active metabolite, were below the limit of quantification, oxymorphone could not be implemented in the model. None of the tested covariates significantly improved the PK model.

Oxycodone AUC and pain scores were correlated; most likely because oxycodone dose was increased when patients reported higher pain scores. Oxycodone, nor-oxycodone and nor-oxymorphone AUC could not be associated with changes in pain scores or adverse events.

Conclusion: This is the first study to successfully describe oxycodone, nor-oxycodone and nor-oxymorphone pharmacokinetics using population PK modelling in a real-world population of cancer patients. Additional research, including more patients and denser collection of PD data, is necessary to further elucidate oxycodone (metabolite) PK/ PD relationships to protect patients with cancer-related pain.

\section{0}

DEVELOPMENT OF A PBPK MODEL OF CYP3A AND CYP2C19 DOWNREGULATION BY INTERLEUKIN-6 AND ESOMEPRAZOLE: PREDICTION OF DISEASE-DRUG AND DRUG-DRUG INTERACTIONS

Camille Lenoir ${ }^{1}$, Amine Niederer ${ }^{1}$, Dr Victoria Rollason ${ }^{1}$,

Prof Jules Alexandre Desmeules ${ }^{1}$, Prof Youssef Daali ${ }^{1}$,

Prof Caroline Flora Samer ${ }^{1}$

${ }^{1}$ Division of Clinical Pharmacology and Toxicology, Department of Anaesthesiology, Pharmacology, Intensive Care and Emergency Medicine, Geneva University Hospitals, Geneva, Switzerland

Introduction: Inter- and intraindividual variability in response to treatment is caused by the interplay of genetic, physiological and environmental factors. This explains the high heterogeneity observed in cytochromes
P450 (CYP) activities. CYP could interact with xenobiotics, leading to drug-drug interaction but they could also contribute to drug-disease interaction, especially with inflammation that downregulates CYP activities via pre- and post-transcriptional mechanisms. Interleukin-6 (IL-6), a key pro-inflammatory cytokine, is mainly responsible of this impact.

Objectives

To develop a physiologically based pharmacokinetic (PBPK) model to foresee the impact of elevated levels of IL- 6 and esomeprazole on CYP3A and CYP2C19 activities.

Methods: Different models were developed to predict the impact of IL-6 on CYP3A and CYP2C19, and results were validated with data from our cohort of elective hip surgery patients whose CYP activities were measured before and after surgery (1).

First, model for IL- 6 was adapted from literature and validated $(1,2)$. Information on CYP3A and CYP2C19 inhibition by IL-6 were obtained from a previously published in vitro study (3). Esomeprazole model was adapted from literature and validated $(4,5)$. Models of midazolam and its metabolite (1-OH-midazolam) were used directly from literature (6). Models of omeprazole and its metabolite (5-OH-omeprazole) were adjusted from literature and further validated $(4,7,8)$. Correction factor was applied to convert drugs concentrations from whole blood to plasma (9).

Results: The model we developed for omeprazole, 5-OH-omeprazole and esomeprazole PK predicted accurately the observed data in healthy subjects $(5,8)$. Indeed, mean AUC ratios for omeprazole/5-OH-omeprazole and esomeprazole were 1.36 and 1.06 respectively. Similarly, simulated concentration-time profiles of IL- 6 versus observed ones in our cohort study of interest were comparable, with AUC ratios of 1.31 (1). The impact of IL- 6 and esomeprazole on the exposure to CYP3A and CYP2C19 probe substrates and respective metabolites were correctly predicted (1). Indeed, the ratio between predicted and observed mean concentrations were $<2$ for all observations.

Conclusion: The impact of IL-6 and esomeprazole on CYP3A and CYP2C19 activities after a hip surgery were correctly predicted with the developed PBPK models.

References

1. Lenoir, C. et al. Clin Pharmacol Ther (2020).

2. Machavaram, K. K. et al. Clin. Pharmacol. Ther. 94, 260-268 (2013).

3. Dickmann, L. J. et al. Drug Metab. Dispos. 39, 1415-1422 (2011).

4. Le Merdy, M. et al. Eur J Drug Metab Pharmacokinet 46, 41-51 (2021).

5. Hassan-Alin, M. et al. Eur J Clin Pharmacol 56, 665-670 (2000).

6. Nguyen, H. Q. et al. Drug Metab Dispos 44, 781-791 (2016).

7. Feng, S. et al. Eur J Clin Pharmacol 71, 617-624 (2015).

8. Bosilkovska, M. et al. Basic Clin. Pharmacol. Toxicol. 119, 284-290 (2016).

9. Bosilkovska, M. et al. Bioanalysis 6, 151-164 (2014).

23

Pharmacokinetics of daridorexant, a dual orexin receptor antagonist, in patients with renal impairment

Phd Benjamin Berger ${ }^{1}$, Clemens Muehlan ${ }^{1}$, Prof. Gernot Klein ${ }^{2}$, Dr Jasper Dingemanse

${ }^{1}$ Idorsia Pharmaceuticals Ltd (Department of Clinical Pharmacology), Allschwil, Switzerland

${ }^{2}$ APEX GmbH, Munich, Germany

Introduction: Daridorexant is a dual orexin receptor antagonist (DORA) in clinical development for insomnia, which has recently been submitted for marketing authorization. Daridorexant is cleared mainly via nonrenal routes, i.e., metabolized by CYP3A4 with fecal excretion being the major path of elimination.

Objectives: The aim of this study was to evaluate the impact of severe renal function impairment (SRFI) on the pharmacokinetics (PK), safety, and tolerability of daridorexant to provide appropriate dosing 
recommendations, as renal function impairment can also affect non-renal elimination mechanisms of drugs.

Methods: This, single-dose, open-label Phase 1 study evaluated the PK of daridorexant in patients with SRFI $(n=8$; creatinine clearance $\leq 30 \mathrm{~mL} /$ min determined by the Cockcroft-Gault equation, not on dialysis) compared to matched healthy subjects $(n=7)$ based on sex, age ( \pm 10 years), and body weight $( \pm 15 \%)$. Daridorexant $25 \mathrm{mg}$ was administered in the morning. Blood sampling for PK evaluations (up to $72 \mathrm{~h}$ post-dose) and safety assessments (clinical laboratory, vital signs, adverse events [AEs], and ECG) were performed regularly. The effect of renal function impairment on plasma protein binding was also assessed.

Results: In patients with SRFI, maximum plasma concentrations (Cmax; geometric mean ratio [GMR] and $90 \%$ confidence interval [CI]: 0.94 [0.60-1.46]), time to reach Cmax (tmax; median difference [90\% CI]: $0.25 \mathrm{~h}[-0.75$ to 0.25$]$ ), and terminal half-life (GMR [90\% CI]: 0.99 [0.66$1.48]$, were virtually unchanged. Area under the plasma concentrationtime curve was slightly higher in patients with SRFI than in healthy subjects (GMR [90\% CI]: 1.16 [0.63-2.12]), while the corresponding apparent total plasma clearance decreased (GMR [90\% CI]: 0.87 [0.471.59]). Furthermore, no difference in plasma protein binding was observed between patients with SRFI and healthy subjects. No safety issue of concern was detected as all AEs were transient and of mild or moderate intensity, and no other treatment-related effects were observed following daridorexant administration in patients with SRFI and healthy subjects.

Conclusion: Based on these observations, PK alterations of daridorexant due to renal function impairment are not considered of clinical relevance and no dose adjustment is necessary for patients with any degree of renal function impairment.

24

Pharmacokinetics of daridorexant, a dual orexin receptor antagonist, in patients with liver cirrhosis

Phd Benjamin Berger ${ }^{1}$, Dr Jasper Dingemanse ${ }^{1}$, Giancarlo Sabattini ${ }^{2}$, Dr Urs Duthaler ${ }^{3}$, Clemens Muehlan ${ }^{1}$, Prof. Stephan Krähenbühl ${ }^{3}$

${ }^{1}$ Idorsia Pharmaceuticals Ltd (Department of Clinical Pharmacology), Allschwil, Switzerland

${ }^{2}$ Idorsia Pharmaceuticals Ltd (Department of Preclinical Drug Metabolism and Pharmacokinetics), Allschwil, Switzerland

${ }^{3}$ University Hospital Basel (Division of Clinical Pharmacology \& Toxicology), Basel, Switzerland

Introduction: Daridorexant is a dual orexin receptor antagonist (DORA) in clinical development for insomnia.

Objectives: As daridorexant is cleared mainly via CYP3A4, the effect of hepatic impairment on its pharmacokinetics (PK), metabolism, and tolerability was evaluated. As sleep disorders are common in such patients, sleep-promoting drugs with better tolerability than currently available would be preferable, a premise which DORAs may fulfill.

Methods: This was a single-dose, open-label, Phase 1 study. Patients with mild (Child [-Pugh] A, $n=8$ ) or moderate (Child $\mathrm{B}, \mathrm{n}=8$ ) liver cirrhosis and matched healthy subjects $(n=8)$ received $25 \mathrm{mg}$ daridorexant orally. Blood samples were collected for $72 \mathrm{~h}$ postdose for PK assessments of daridorexant and 3 major metabolites.

Results: Compared to healthy subjects, patients showed a decrease in total daridorexant area under the plasma concentration-time curve (AUC) and maximum plasma concentration (Cmax) with a geometric mean ratio (GMR, 90\% confidence interval [CI]) of $0.51(0.28$ $0.92)$ and $0.50(0.35-0.72)$ in Child A and $0.74(0.39-1.41)$ and 0.42 (0.29-0.60) in Child B patients, respectively. Furthermore, the median time to reach Cmax (tmax) was slightly delayed (1.0 h [90\% CI: $0.0-2.0]$ in Child A, $0.5 \mathrm{~h}$ [0.0-1.5] in Child B patients), while for Child B patients, a doubling in terminal half-life $\left(t^{1 / 2}\right)$ was observed (2.09 [1.32-3.30]). Considering the high plasma protein binding $(>99 \%)$ and a 1.9-2.3-fold increase in the unbound fraction in patients with liver cirrhosis, the PK of unbound daridorexant were also assessed. Compared to healthy subjects, Child B patients had a higher AUC (1.60 [0.93-2.73]), a lower apparent plasma clearance $(0.63[0.37-1.07])$, and the same doubling in $t^{1 / 2}$ as total daridorexant, while Cmax was unchanged. Unbound daridorexant PK in Child A patients did not differ on PK from healthy subjects. All treatment-emergent adverse events were transient and of mild/moderate intensity with no other treatment-related effects apparent.

Conclusion: No safety issue of concern was detected following administration of $25 \mathrm{mg}$ daridorexant. Moderate liver cirrhosis causes impaired hepatic clearance of unbound daridorexant, which prolongs $t^{1} / 2$. Therefore, while not required in Child A patients, a dose reduction is recommended in Child B patients, whereas daridorexant should be avoided in Child C patients.

25

The 1 $\beta$-hydroxy-deoxycholic acid to deoxycholic acid urinary metabolic ratio: an endogenous CYP3A metric?

Gaëlle Magliocco ${ }^{1}$, Prof Jules Desmeules ${ }^{1}$, Dre Marija Bosilkovska ${ }^{1}$, Prof Aurélien Thomas ${ }^{2}$, Prof Youssef Daali ${ }^{1}$

${ }^{1}$ Geneva University Hospitals, Clinical Pharmacology and Toxicology, Geneva, Switzerland

${ }^{2}$ Lausanne University Hospitals, Geneva University Hospitals, Forensic Toxicology and Chemistry Unit,, Lausanne-Geneva, Switzerland

Introduction: The prescription of cytochrome P450 3A (CYP3A) drug substrates is often challenging for physicians due to the high basal inter-individual variability in the activity of these enzymes. The development of tools for real-time phenotyping of CYP3A is therefore necessary to enhance therapeutic effectiveness and drug safety. In this context, bile acids are potential endogenous markers of CYP3A since their concentration in humans is partly regulated by these enzymes through biotransformation into less lipophilic compounds. In particular, the secondary bile acid deoxycholic acid (DCA) was found to be specifically metabolized by CYP3A into $1 \beta$-hydroxy-deoxycholic acid $(1 \beta-\mathrm{OH}-\mathrm{DCA})$ using recombinant enzymes.

Objectives: The aim of this study was therefore to assess the potential use of the $1 \beta-O H-D C A / D C A$ urinary metabolic ratio (UMR) as a CYP3A metric in healthy subjects. In particular, we evaluated the relationship between the $1 \beta-\mathrm{OH}-\mathrm{DCA} / \mathrm{DCA} \mathrm{UMR}$ and the validated CYP3A probe, midazolam (MDZ), under constitutive conditions and with the influence of CYP3A inhibitors/inducer.

Methods: MDZ $1 \mathrm{mg}$ was administered per os in ten male healthy volunteers at three sessions as the reference probe drug to measure CYP3A activity: alone (control session), after pre-treatment with fluvoxamine $50 \mathrm{mg}$ ( $12 \mathrm{~h}$ and $2 \mathrm{~h}$ prior to $\mathrm{MDZ}$ administration) and voriconazole $400 \mathrm{mg}$ ( $2 \mathrm{~h}$ before $\mathrm{MDZ}$ administration) (inhibition session), and after a 7-day pre-treatment with the inducer rifampicin $600 \mathrm{mg}$ (induction session). The $1 \beta-\mathrm{OH}-\mathrm{DCA} / \mathrm{DCA}$ UMR was measured at each session and correlations with MDZ metrics, including oral clearance $(\mathrm{CL})$, were established.

Results: At baseline, the $1 \beta-\mathrm{OH}-\mathrm{DCA} / \mathrm{DCA}$ UMR correlated significantly with oral MDZ CL $(r=0.652, P=0.041)$ and $\mathrm{Cmax}(\mathrm{r}=-0.652$, $\mathrm{P}=0.041$ ). In addition, modulation of CYP3A was reflected in the $1 \beta-\mathrm{OH}-\mathrm{DCA} / \mathrm{DCA}$ UMR after intake of rifampicin (induction ratio $=11.4, \mathrm{P}<0.01)$. During the inhibition session, a non-significant $22 \%$ decrease in $1 \beta-\mathrm{OH}-\mathrm{DCA} / \mathrm{DCA}$ was observed $(\mathrm{P}=0.275)$.

Conclusion: Changes in the $1 \beta-\mathrm{OH}-\mathrm{DCA} / \mathrm{DCA}$ UMR can be used as a surrogate for MDZ, notably, following multiple doses of rifampicin, a CYP3A inducer. Additional studies, particularly involving CYP3A inhibition for longer period (in order fully eliminate baseline levels of $1 \beta-\mathrm{OH}-\mathrm{DCA}$ and DCA beforehand) and larger sample sizes, are needed to evaluate the endogenous $1 \beta-\mathrm{OH}-\mathrm{DCA} / \mathrm{DCA}$ metric as a suitable CYP3A biomarker. 
38

Pharmacokinetics and Safety of Atorvastatin Co-administered with Tegoprazan or Vonoprazan in Healthy Subjects

Dr. Seokuee Kim ${ }^{1}$, Haerim Jang ${ }^{1}$, Hyosook Seo ${ }^{1}$, Dr. Bongtae Kim ${ }^{1}$, $\overline{\text { Dr. Jae-Wook } \mathrm{Ko}^{2}}$, Dr. Jung-Ryul Kim ${ }^{3}$

${ }^{1}$ Division of Clinical Development, HK inno.N Corp., Seoul, Republic of Korea

${ }^{2}$ Department of Clinical Pharmacology and Therapeutics, Samsung Medical Center, Seoul, Republic of Korea

${ }^{3}$ Department of Clinical Pharmacology and Therapeutics, Samsung Medical Center \& Department of Clinical Research Design \& Evaluation, SAIHST, Sungkyunkwan University, Seoul, Republic of Korea

Introduction: Tegoprazan, a potassium-competitive acid blocker (P$\mathrm{CAB}$ ), is a novel acid suppressant with different mechanism of action from the proton pump inhibitors (PPIs). It is approved for treatment of diseases related to gastric acid secretion. Vonoprazan is also a P-CAB, which is a potential CYP3A4 inhibitor. From Korean market data in 2018, atorvastatin ranked the 7th most frequently co-prescribed agent with PPIs. In this regard, it was necessary to confirm the effects of tegoprazan and vonoprazan on pharmacokinetic (PK) profile of atorvastatin.

Objectives: We conducted the clinical trial to evaluate the effects of tegoprazan and vonoprazan on the PK and safety profile of atorvastatin following co-administration of tegoprazan or vonoprazan with atorvastatin in healthy subjects.

Methods: An open-label, randomized, multiple-dose, replicated crossover study to evaluate drug interaction following co-administration of tegoprazan or vonoprazan with atorvastatin was conducted in healthy Korean subjects. Atorvastatin $(40 \mathrm{mg})$ was administered orally with or without the tegoprazan $(50 \mathrm{mg})$ or vonoprazan $(20 \mathrm{mg})$. Blood PK samples were collected up to 24 hours in steady-state. Plasma atorvastatin concentrations were measured with validated liquid chromatography/ tandem mass spectrometry. The PK parameters of atorvastatin were determined by noncompartmental analysis.

Results: Twenty-eight subjects were included in PK analysis. Geometric least-square mean ratio and $90 \%$ confidence intervals of the steady-state maximum concentration and area under the plasma concentration versus time curve over dosing interval of atorvastatin with tegoprazan were 1.09 (0.97-1.23) and 1.03 (0.98-1.08), respectively. And those of atoravstatin with vonoprazan were 1.17 (1.04-1.32) and 1.28 (1.22-1.34), respectively. No serious adverse event was observed. Although the incidence of adverse event (AE) was not statistically significantly different between atorvastatin with or without tegoprazan ( $p$-value $=0.91$ ), but it was statistically significantly different between atorvastatin with or without vonoprazan ( $\mathrm{p}$-value $=0.01$ ).

Conclusion: In healthy subjects, co-administration of atorvastatin and vonoprazan led to increases in systemic exposure of atorvastatin and the $\mathrm{AE}$ incidence, while such drug interaction were not observed in coadministration of atorvastatin and tegoprazan.

\section{9}

Pharmacokinetic interactions between tegoprazan and naproxen, aceclofenac and celecoxib in healthy Korean subjects

Dr. Seol Ju Moon ${ }^{1}$, Naree Shin ${ }^{2}$, MinJa Kang ${ }^{2}$, Bongtae Kim², Min-Gul Kim ${ }^{1,3,4}$

${ }^{1}$ Center for Clinical Pharmacology and Biomedical Research Institute, Jeonbuk National University Hospital, Jeonju-si, Republic of Korea,

${ }^{2}$ Division of Clinical Development, HK inno.N Corp., Seoul, Republic of Korea

${ }^{3}$ Research Institute of Clinical Medicine of Jeonbuk National University, Jeonju-si, Republic of Korea

${ }^{4}$ Department of Pharmacology, School of Medicine, Jeonbuk National University, Jeonju-si, Republic of Korea
INTRODUCTION: Tegoprazan is a potassium-competitive acid blocker used for gastric acid suppression, and may be used in patients who need non-steroidal anti-inflammatory drug (NSAID) treatment, in order to reduce the risk of gastric ulcer caused by NSAIDs administration. OBJECTIVES: This study aimed to evaluate the pharmacokinetic interaction between tegoprazan and commonly used NSAIDS, namely naproxen, aceclofenac and celecoxib.

METHODS: An open-label, 3-cohort, randomized, multiple-dose, 3way crossover study was conducted in healthy subjects. In cohort 1 , tegoprazan (50 mg tablet, once daily) and naproxen (500 mg tablet, twice daily) were administered separately or concurrently for 7 days in each period. In cohort 2, tegoprazan and aceclofenac (100 mg tablet, twice daily) were administered separately or concurrently for 7 days in each period. In cohort 3, tegoprazan and celecoxib (200 mg capsule, twice daily) were administered separately or concurrently for 7 days in each period. Pharmacokinetic blood samples were collected up to $24 \mathrm{~h}$ after the last dose.

RESULTS: Seventeen subjects from cohort 1, 16 subjects from cohort 2, and 13 subjects from cohort 3 were included in the pharmacokinetic analysis. In cohort 1, the geometric least squares mean ratios (90\% confidence intervals, CIs) for AUC $\tau$ and Css,max were 1.01 (0.91-1.12) and $0.99(0.83-1.17)$ for tegoprazan, and $1.00(0.97-1.03)$ and $1.04(0.99-$ 1.09) for naproxen, respectively. The values in cohort 2 were 1.03 (0.93-1.13) and $0.94(0.86-1.04)$ for tegoprazan, and $1.06(1.00-1.12)$ and 1.31 (1.08-1.60) for aceclofenac, respectively. The values in cohort 3 were $1.01(0.86-1.18)$ and $1.02(0.87-1.19)$ for tegoprazan, and 1.08 (0.96-1.22) and $1.18(0.97-1.43)$ for celecoxib, respectively.

CONCLUSION: Changes in the maximum aceclofenac or celecoxib concentrations were observed after concurrent administration with tegoprazan, which were considered mainly due to the pharmacodynamic effect of tegoprazan. Because the systemic drug exposure (AUC $\tau$ ) was not changed after concurrent administration of any 3 NSAIDs with tegoprazan, the increase in aceclofenac or celecoxib Css, max when administered with tegoprazan would not be clinically significant in practice.

53

A preliminary population pharmacokinetic model for remdesivir and its metabolites based on published mean values from healthy subjects

$\underline{\text { Ahmed Abouellil }^{1,2}}$, Univ.-Prof. Dr. med. Uwe Fuhr ${ }^{2}$,

Muhammad Bilal $^{2}$

${ }^{1}$ Immunosensation cluster of excellence, University Hospital Bonn, Bonn, Germany

${ }^{2}$ Faculty of Medicine and University Hospital Cologne, Center for Pharmacology, Department I of Pharmacology, University of Cologne, Cologne, Germany

Introduction: Remdesivir is an inhibitor of the viral RNA-dependent RNA polymerase, and is one of the first drugs to be authorized for the treatment of COVID-19. However, due to its relative novelty and the accelerated authorization process pharmacokinetic data is sparse.

Objectives: The objective of this project was to develop a structural population pharmacokinetic model and point estimates for PK parameters for remdesivir and its major metabolites which can be quantified in human plasma, i.e. GS-704277 and GS-441524.

Methods: Getdata (S. Fedorov, getdata-graph-digitizer.com) was used to retrieve mean data from graphs of the published study that evaluated safety and pharmacokinetics of single and multiple ascending intravenous doses of remdesivir (3-225 mg) in healthy subjects (Humeniuk R et al. Clin Transl Sci. 2020 Sep;13(5):896906). Criteria for eligibility were: male or female, age $18-55$ years, body mass index $18-30 \mathrm{~kg} / \mathrm{m} 2$. Population PK models were developed by standard methods using non-linear mixed effect modelling (NONMEM and MONOLIX). 
Results: The model which described PK data best had two compartments for remdesivir and both metabolites, respectively, with metabolism reflected by transfer from one central compartment to the next, and with an additional transfer from the peripheral remdesivir compartment to the peripheral GS-704277 compartment. Elimination was assumed to occur from the central compartments of remdesivir and both metabolites. "Intermean variability" was allowed for about half of the parameters. Goodnessof-fit plots and visual predictive checks indicated that the model was suitable to describe the mean concentration vs. time profiles of remdesivir and both metabolites at all doses tested. Point estimates for central (peripheral) volumes of distribution for remdesivir, GS-704277 and GS441524 were $4.89 \mathrm{~L}(46.5 \mathrm{~L}) ; 96.4 \mathrm{~L}(8.64 \mathrm{~L})$; and $26.2 \mathrm{~L}(66.2 \mathrm{~L})$, respectively. The estimated elimination clearances of remdesivir, GS704277 and GS-441524 reached $18.1 \mathrm{~L} / \mathrm{hr}, 36.9 \mathrm{~L} / \mathrm{hr}$, and $4.74 \mathrm{~L} / \mathrm{hr}$, respectively.

Conclusions: This preliminary model is a first step to describe and simulate exposure for remdesivir and its metabolites in healthy volunteers by a compartmental approach, with the prespective to predict concentrations in other populations.

\section{Poster Session 1b - Research/professional development}

\section{4}

ACCELERATE: A Novel Study Design Used in Clinical Research of Castleman Disease

Dr Martin Lukac ${ }^{1}$, BS Johnson Khor $^{2}$, BA Freda Coren ${ }^{2}$, $\overline{\text { BS Reece Williams }}^{2}$, MS Sheila Pierson ${ }^{2}$, MD, MBA, MSc David Fajgenbaum ${ }^{2}$

${ }^{1}$ Castleman Disease Collaborative Network, Prague, Czech Republic,

${ }^{2}$ University of Pennsylvania, Department of Medicine, Philadelphia, USA

Introduction: Castleman disease (CD) is a group of rare disorders, characterized by lymphadenopathy with substantial variability in the symptomatology. Fragmentation of data and biospecimens, and treatment variability present challenges to describe the natural history of CD and new treatment strategies.

Objectives: Innovative patient-powered study design was created to advance the understanding of the natural history and real-world treatment in CD.

Methods: ACCELERATE registry consists of the novel patient-powered arm (PPA) in the US, and the traditional physician-directed arm (PDA), at 9 sites in six EU countries. In the PDA, the site physician confirms inclusion criteria (CD pathology report) and patients provide informed consent to extract their medical data. In the PPA, patients provide informed consent electronically and enroll themselves via a web-based portal. After CD pathology report is obtained and eligibility is confirmed, the ACCELERATE Registry Team requests patient medical records from institutions providing CD-related care. A trained data analyst manually extracts all data elements from medical records into the central database. Each case is then reviewed with the study's principal investigator.

Results: During 42 months of enrollment (started Q4 2016), 468 patients had enrolled (PPA: 372, PDA: 96), and of those, 365 were eligible (PPA: 271, PDA: 94). The mean age at diagnosis is 40.2 (SD: 16.3) years (range: $1-80)$, with females composing 55\% (201/365). Of the 271 PPA eligible patients, 59 self-uploaded pathology report during enrollment (22\%). For the rest median (interquartile range $[\mathrm{IQR}]$ ) time to receive pathology report was 25 (3-67) days. The remaining time until receipt of retrospective data was 145 (44-282) days. The median (IQR) length of a patient's medical record is $531(274-1,041)$ pages. Patients in the PPA have a median (IQR) of 683 (323-1334) clinical, laboratory, and imaging data elements entered, compared to 37 (19-69) data elements in PDA. We have identified 62 unique $C D$ treating medications used in a real-life practice, with prednisone, rituximab and siltuximab being the most frequently collected.

Conclusion: Novel design of ACCELERATE has improved collection of patient data and tissue biospecimens, which have been used in translational and clinical studies and treatment guidelines. For further details please see full manuscript Pierson et al., Cell Reports Medicine 1, 100158, 2020

5

Implementation of Physician Clinical Pharmacologist Services in the Lithuanian University of Health Sciences Hospital of Kaunas

Egle Karinauske $^{1}$, Dr. Rima Jurate Gerbutaviciene ${ }^{1,2}$,

Aurimas Peckauskas ${ }^{1,2}$, Prof. Diana Zaliaduonyte ${ }^{1,2}$

${ }^{1}$ Lithuanian University Of Health Sciences Hospital Of Kaunas, Kaunas, Lithuania

${ }^{2}$ Lithuanian University Of Health Sciences, Kaunas, Lithuania

Introduction and objectives: The role of physician clinical pharmacologist (PCP) is not yet systematically developed in Lithuania. According to current data, there are only 7 PCPs who attended post-graduate clinical pharmacology residency program and gained PCP's profession in Lithuania. For the first time, in August, 2020, one PCP started working at Lithuanian University of Health Sciences Hospital of Kaunas. The purpose of PCP is to rationalize prescribing of antimicrobial drugs, deprescribing of any drugs and other. The main objective of our study was to analyze the need of PCP's services.

Methods: This perspective cohort study was conducted during the six-month period (August 14th, 2020 till January 31st, 2021). The clinicians approached PCP for a consultation for managing an index patient. We registered all PCP's consultations, their purpose (for example, drug or dosing information, prescribing or de-prescribing drugs) and later followed up with the patient to see if the recommended action was taken. The results are presented as descriptive statistics.

Results: During the six-month period PCP was approached by the physician specialist for 210 consultations for 152 patients. Patient mean age was 71.09 ( $\min : \max 9.95$ ) years. The most common purpose of the consultations was to prescribe/ de-prescribe antimicrobial medicines $(86.2 \%, 181$ out of 210$)$, and other (pain management, prescribing or de-prescribing of anticoagulants, assessment of suspected adverse drug reactions, drug information of preparation of solutions) $(13.8 \%, 29$ out of 210$)$. Fifty percent of the consultations were provided to physicians working at Internal diseases unit, $29.5 \%$ at Cardiology unit, $10 \%$ Neurology unit and $10.5 \%$ at Psychiatry unit, Intensive care unit and Surgical unit. According to the data we collected, more than $95 \%$ (201 out of 210 consultations) of clinicians followed the pharmacotherapy advice recommended by PCP in their patient management.

Summary/ Conclusions: Although, the profession of PCP is quite new in Lithuania, our study shows that there is a need of PCP services in Lithuanian University of Health Sciences Hospital of Kaunas. Data presents that mostly therapeutic units (Internal diseases unit, Cardiology unit and Neurology unit) cooperates with PCP. Our vision is to expand the range of clinical pharmacology services in our hospital.

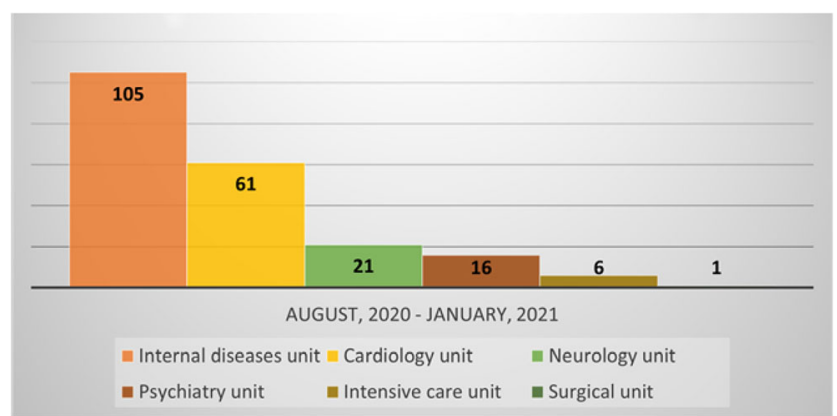


13

NEW STRATEGIES FOR MONITORING ACTIVITY IN CLINICAL TRIALS DURING THE COVID-19 PANDEMIC

Phd Lucia Lavin-alconero ${ }^{\mathbf{1 , 2}}$, MD Rita Nogueiras-Alvarez ${ }^{2}$, Lic, MSc Tatiana Fernandez-Lanas ${ }^{1,2}$, MSc, PhD Jose Carlos Garrido-Gracia ${ }^{3}$, Lic, MSc Laura Burunat-Ruesgas ${ }^{4}$, Lic, MSc Miriam Cervino-Rodriguez, Lic, MSc Mireia Hernandez-Hernandez ${ }^{6}$, Lic, MSc Jose Manuel Nicolas de la Puente ${ }^{2}$, MD, MSc, PhD Mar García-Saiz ${ }^{1,2}$

${ }^{1}$ IDIVAL, SANTANDER, SPAIN

${ }^{2}$ Hospital Universitario Marqués de Valdecilla, SANTANDER, SPAIN

${ }^{3}$ IMIBIC-Hospital Reina Sofía, CORDOBA, SPAIN

${ }^{4}$ IDIBAPS-Hospital Clinic Barcelona, BARCELONA, SPAIN

${ }^{5}$ FIISC-Hospital de Canarias, TENERIFE, SPAIN

${ }^{6}$ INCLIVA-Hospital Clinico de Valencia, VALENCIA, SPAIN

Introduction: The monitoring of a clinical trial (CT) guarantees the rights, safety and well-being of the subjects, as well as the robustness and reliability of the data obtained. Its application is essential for compliance with good clinical practice and current legislation on CT.

At the beginning of the pandemic, the Spanish Agency of Medicines and Health Products (AEMPS) published a document of exceptional measures applicable to CT's to manage the problems derived from COVID19 , to guarantee the CT activity and the traceability of the actions implemented, together with the action plans for notifications to the Ethics Committees and AEMPS.

On-site monitoring has been partially/totally restricted during the pandemic, and the monitoring plans have been adapted to continue providing the necessary support to centers and sponsors. Likewise, the research team has modified its clinical practice to comply with health recommendations, maintaining the quality of care and research activity.

Objectives: identify new strategies for monitoring that emerged during the COVID-19 pandemic.

Methods: An online questionnaire was conducted with the google form tool with 53 ad hoc items, with open and closed questions, between July and August 2020 (4 months after the beginning of the pandemic declaration in Spain). This questionnaire was sent to coordinators, monitors and project managers, from public research institutes and private companies. Results: We obtained data from 110 professionals (57.27\% from Public system and $42.72 \%$ from private company). The survey shows a change in the face-to-face visits to remote visits in $30 \%$ of the cases and with a total of $66.4 \%$ of the visits performed remote. The new monitoring strategies are based on: New monitoring models and Changes in access to source data. In both cases statistically significant differences are found between private and public settings.

Regarding to medication management, a radical change was observed: before pandemic the $90.9 \%$ of the survey responders did not work with home delivery afterwards the $54.5 \%$ of them reported to have begun doing it.

Conclusion: The adaptation of monitoring using new strategies has allowed the continuity of follow-up and/or control of clinical data by the personnel involved. This has made it possible to maintain quality and optimize resources without overloading the researcher's healthcare activity.

\section{3}

A cookbook for estimating treatment duration from databases of routinely collected electronic health data participating in international pharmacoepidemiologic studies

Dr Judit Riera-Arnau ${ }^{1}$, Nicolas H Thurin ${ }^{2}$, Giuseppe Roberto ${ }^{3}$,

Patrick Souverein ${ }^{4}$, Rosa Gini $^{3}$, Romin Pajouheshnia ${ }^{4}$

${ }^{1}$ Clinical Pharmacology Service, Vall d'Hebron University Hospital, Autonomous University of Barcelona, Barcelona, Spain

${ }^{2}$ Bordeaux PharmacoEpi, INSERM CIC1401, Université de BORDEAUX, Bordeaux, France
${ }^{3}$ Agenzia regionale di sanità della Toscana, Osservatorio di epidemiologia, Florence, Italy

${ }^{4}$ Division of Pharmacoepidemiology and Clinical Pharmacology, Utrecht Institute for Pharmaceutical Sciences, Utrecht University, Utrecht, The Netherlands

Introduction: Information required to calculate the dispensed number of days of treatment (NoDT) of individual prescriptions or dispensing is often incompletely recorded and may vary substantially across different sources of routinely collected electronic health data (EHD). As a result, different assumptions on treatment dose and duration might be necessary according to the information that is available. This raises concerns over heterogeneity in the way that exposure to a medicine is defined and reported in pharmacoepidemiologic studies using EHD.

Objectives: To i) standardize the terms and variables needed when calculating NoDT across different EHD, and ii) define a series of assumptions and calculation options that can be adapted to different EHD depending on the availability of prescription, dispensing, administrative and product information.

Methods: We compiled a list of common information on medicinal products that may be captured (or not) in EHD. Guidelines of European standard terms for medicines were reviewed and a "cookbook" of unique algorithms to calculate NoDT, i.e. recipes, was derived.

Results: Variables describing medicinal products used to estimate NoDT were established and terms harmonized, such as, amount of active principle per day, basic dose forms to be administered to the patient or options for administration routes. Four main recipes with four variants were created for NoDT calculation on the basis of the variables included in a database, ordered from a complete availability of posology prescribed and dispensation data, to only knowing the medicinal product name (see attached Table). In the case that only the product name was known, the amounts of medicinal product in the box were extracted from the product name and duration estimated through prescribed (PDD) or defined daily doses (DDD). Model examples of how these calculations can be made with different types of medicinal products (tablets, combinations, syrup, topicals, vials, inhalations, powder, etc) were created.

Conclusion: There is heterogeneity in the information required to estimate NoDT, which may lead to biases due to the definition of exposure when cross-national studies are performed. We provide terminology and rules to harmonize available information to calculate NoDT, to enhance the comparability of exposure definitions across data sources.

\begin{tabular}{|c|c|}
\hline Order & Number of Days of Treatment calculation recipes \\
\hline 1 & (Number of forms per box) /(Prescribed forms per day) \\
\hline 2 & $[($ Dosage amount of active principle per box $)] /[($ Amount per form $)] \times$ (Prescribed forms per day $)$ \\
\hline 3 & {$[($ Amount per form) $\times$ (Number of forms per box $)] /$ (Prescribed amount per day) } \\
\hline 4 & (Dosage amount of active principle per box) / (Prescribed amount per day) \\
\hline 5 & Substitute "Prescribed amount per day" in RECIPE 3 by PDD \\
\hline 6 & Substitute "Prescribed amount per day" in RECIPE 4 by PDD \\
\hline 7 & Substitute "Prescribed amount per day" in RECIPE 3 by DDD \\
\hline 8 & Substitute "Prescribed amount per day" in RECIPE 4 by DDD \\
\hline
\end{tabular}

46

A randomized controlled clinical trial comparing alcohol and alcohol mixed with energy drinks under a binge-drinking pattern Dr Clara Pérez-Mañá ${ }^{1,2}$, MD Olga Hladun ${ }^{1,2}$, PhD, MD Esther ${ }_{\text {Papaseit }}^{1,2}$, PhD student Lourdes Poyatos ${ }^{1,2}$, RN Soraya Martin ${ }^{1}$, $\mathrm{PhD}, \mathrm{MD}$ Ana Maria Barriocanal ${ }^{1,2}$, MD Lucía Martínez $^{3}$, $\mathrm{PhD}, \mathrm{MD}$ Magí Farré ${ }^{1,2}$

${ }^{1}$ Department of Clinical Pharmacology, Hospital Universitari Germans Trias i Pujol and Institut de Recerca Germans Trias i Pujol (IGTP), Badalona, Spain 
${ }^{2}$ Department of Pharmacology, Therapeutics and Toxicology, Universitat Autònoma de Barcelona (UAB), Cerdanyola del Vallès, Spain

${ }^{3}$ Department of Clinical Pharmacology, Hospital de la Paz, Madrid, Spain

Introduction: Consumption of alcohol mixed with energy drinks (ED) has increased mainly among young people. Binge-drinking is a pattern of drinking that brings blood alcohol concentration to $\geq 80 \mathrm{~g} / \mathrm{dl}$ (achieved in men consuming $\geq 5$ drinks or women with $\geq 4$ drinks in 2 hours). The relevance of gender in acute effects of alcohol when it is combined with ED using this risky pattern of consumption has been poorly studied.

Objectives: To study gender differences in concentrations and acute effects of alcohol combined with ED administered experimentally in a binge-drinking pattern.

Methods: A randomized clinical trial is being conducted in 26 healthy volunteers (1:1) with four treatment conditions: alcohol+ED, alcohol+placebo of ED, placebo of alcohol+ED and both placebos. Alcohol $70 \mathrm{~g}$ in men and $55 \mathrm{~g}$ in women was mixed with $750 \mathrm{ml}$ of ED in men and $589 \mathrm{ml}$ in women. Total volume was divided into 6 equal fractions administered one every 15 min along $80 \mathrm{~min}$. Subjective effects, driving-related skills and alcohol concentrations were assessed along an 8-hours period.

Results: Preliminary results in 10 volunteers ( 6 men: 4 women), with an average age of 22.7 years and weight of $72.3 \mathrm{~kg}$, have shown mean peak breath alcohol concentrations of $0.46 \mathrm{mg} / \mathrm{L}$, correponding to $>80 \mathrm{mg} / \mathrm{dl}$ in blood $(0.49 \mathrm{mg} / 1$ in men vs. $0.42 \mathrm{mg} / 1$ in women). Subjects reported moderate drunkenness feelings (mean peak of 42 and $45.7 \mathrm{~mm}$ in men and 34.8 and $53.3 \mathrm{~mm}$ in women with each alcoholic condition). Mean reaction time increases with alcohol $(45.8$ or $75.7 \mathrm{~ms})$, depending on alcohol mixer.

Conclusion: Breath alcohol concentrations are slightly higher in men, as it is the dose, although both genders have similar subjective and psychomotor effects. Women seem to have less tolerance to the mix of alcohol and ED consumed in a binge-drinking pattern.

Acknowledgements: Ministerio de Sanidad, Política Social e Igualdad, Plan Nacional Sobre Drogas (PNSD 2018 I037 and PNSD 2016 I024), Instituto de Salud Carlos III (Red de Trastornos Adictivos ISCIII-FEDER RD16/0017/0003, Predoctoral grants FI18/00179 Lourdes Poyatos) and AGAUR Gencat Suport Grups Recerca ( 2017 SGR 316).

50

Challenges of a tertiary hospital's ethics committee in evaluating research during the COVID-19 pandemic. The CEICOV study

Elena Guillen $^{1}$, Judit Riera-Arnau ${ }^{1,2}$, Carla Sans-Pola ${ }^{1,2,3}$, Esther Cucurull $^{1,3}$, María Luján ${ }^{1,3}$, Valentina Balasso ${ }^{3,4}$, Esperanza Zuriguel $^{3,5}$, Mireia Navarro ${ }^{3}$, Mireia Tomàs ${ }^{3}$, Lina M. Leguízamo-Martínez ${ }^{1,2,3}$, Francesca Filippi-Arriaga ${ }^{1}$, Alexis Rodríguez ${ }^{1,3}$

${ }^{1}$ Clinical Pharmacology Department, University Hospital Vall D’hebrón, Barcelona, Spain

${ }^{2}$ Universitat Autònoma de Barcelona, Barcelona, Spain

${ }^{3}$ Vall d'Hebron Research Institute (VHIR), Barcelona, Spain

${ }^{4}$ Preventive Medicine and Epidemiology Department, Hospital Universitari Vall d'Hebron, Barcelona, Spain

${ }^{5}$ Nursing Management, Hospital Universitari Vall d'Hebron, Barcelona, Spain

Background: The COVID-19 pandemic has led to an increase in research activity worldwide. The Vall d'Hebron University Hospital (VH) Research Ethics Committee (VH-REC) adapted its procedures to give out opinion in a short period of time. We aimed to describe the characteristics of the evaluated studies during the first wave of the pandemic.

Methods: Clinical trials (CT), post-authorization studies (PAS) and research projects (RP) related to COVID-19 evaluated by the VH-REC, from 16th of March to 21st of June of 2020 were included. The analysis was performed with RStudio through usual descriptive methods.
Results: 157 studies were evaluated: $10 \mathrm{CT}, 16 \mathrm{PAS}$ and $131 \mathrm{RP}$, in $25 \mathrm{bi}-$ weekly telematic meetings. 94\% (148) had independent promoters. The main services involved were Infectious Disease, Intensive Medicine and Pneumology. 45\% (88) were unicentric and 86\% (135) national. 10\% (16) studied healthcare professionals, $10 \%$ immunosuppressed patients and a $9 \%$ pregnancy and neonates, with no overlapping. The main objective of CT and PAS studies was to test efficacy and safety, while for RP, it was to describe incidence or prevalence of a certain outcome in COVID-19 patients. The median time for the evaluation of the protocols was 3 days. The final opinion was favorable in 93\% (146) and unfavorable in 2\% (3). $58.6 \%$ (92) required further clarifications, of which 8 [IQR (3-14)] did not respond. The most frequent causes were aspects of the patient's informed consent sheet, data protection and biological samples.

Conclusions: The expansion of clinical research related to COVID-19 resulted in an increase of VH-REC activity, which had to adapt rapidly in order to give out the opinion in a short period of time. The included protocols show a special concern on certain interest groups and are less likely to be commercially promoted. The information here shown could be useful to compare research trends with the pre-pandemic period, or the evolution of this tendency when what is known about the COVID-19 disease evolves.This project's registry is being extended to other tertiary hospitals from our setting and a follow-up of the protocols already included is underway.

56

Acute pharmacological effects and oral fluid concentrations of methylone and MDMA in humans: An observational-naturalistic study

Md, Phd Esther Papaseit ${ }^{1,2}$, PhD Student Lourdes Poyatos ${ }^{1,2}$, PhD Eulàlia Olesti ${ }^{1,2}, \mathrm{MD}$, PhD Clara Pérez-Mañá ${ }^{1,2}$

$\mathrm{PhD}$ Mireia Ventura ${ }^{4}, \mathrm{MD}$ Marc Grifell ${ }^{5}, \mathrm{MD}, \mathrm{PhD}$ Marta Torrens ${ }^{5}$, MD, PhD Rafael de la Torre ${ }^{5}, \mathrm{MD}, \mathrm{PhD}$ Magí Farré ${ }^{1,2}$

${ }^{1}$ Department of Clinical Pharmacology, Hospital Universitari Germans Trias i Pujol and Institut de Recerca Germans Trias i Pujol (HUGTiPIGTP) and Department of Pharmacology, Therapeutics and Toxicology, Badalona, Spain

${ }^{2}$ Department of Pharmacology, Therapeutics and Toxicology, Universitat Autònoma de Barcelona (UAB), Barcelona, Spain, Barcelona, Spain

${ }^{3}$ Integrative Pharmacology and Systems Neuroscience Research Group, Neurosciences Research Program, Hospital del Mar Medical Research Institute (IMIM) and Universitat Pompeu Fabra (CEXS-UPF), Barcelona, Spain

${ }^{4}$ Energy Control, Associació Benestar i Desenvolupament, Barcelona, Spain

${ }^{5}$ Department of Psychiatry and Forensic Medicine, Institut de Neuropsiquiatria i Adiccions (INAD), Universitat Autònoma de Barcelona (UAB), Barcelona, Spain

Introduction: Methylone (3,4-methylenedioxy-N-methylcathinone) also known as "bk-MDMA" is one of the most commonly used synthetic cathinones. It is a ring-substituted beta-keto-amphetamine closely related to ecstasy (MDMA, 3,4-methylenedioxymethamphetamine). Despite its appearance in the market years ago, the available data about its acute pharmacological effects and pharmacokinetics in humans is very limited and based on surveys and cases of intoxication attended in emergency room.

Objectives: An observational-naturalistic study was conducted to evaluate the acute pharmacological effects of methylone in recreative drug users in comparison to MDMA in a natural setting.

Methods: Fourteen healthy recreative poly-drug users (10 males, 4 females) with reported experience with different psychostimulants and hallucinogens participated in the study. They selected their own methylone or MDMA doses based on their preference. Eight subjects self-ingested a single oral dose of methylone [mean $187.5 \mathrm{mg} ; 100 \mathrm{mg}$ (1 male), $150 \mathrm{mg}$ (1 male, 1 female), $200 \mathrm{mg}$ ( 2 males, 2 females), $300 \mathrm{mg}$ ( $1 \mathrm{male}$ )] and six 
subjects self-ingested a single oral dose of MDMA [mean dose $87.5 \mathrm{mg}$; $75 \mathrm{mg}$ ( 2 males, 1 female), $100 \mathrm{mg}$ ( 3 males)].

Study variables included vital signs (blood pressure, heart rate, temperature) and subjective effects using visual analogue scales, ARCI-49 item short form, and VESSPA questionnaire. Additionally, oral fluid samples were collected to determine concentrations. All of them were assessed at $0,1,2$ and 4 hours.

Results: Methylone and MDMA produced marked increases in vital signs and subjective effects related to stimulation and euphoria (VAS "intensity", "stimulated", "high", ARCI-MBG-euphoria) and induced increases in the scores of other ARCI and VESSPA questionnaires. Methylone produced higher effects on heart rate and lower increase in VAS scores in comparison to MDMA. Similar scores in ARCI and VESSPA questionnaires were reported for both substances.

Oral fluid peak concentrations after methylone and MDMA were 2936.37 $\mathrm{ng} / \mathrm{mL}$ and $15514 \mathrm{ng} / \mathrm{mL}$, respectively at $2 \mathrm{~h}$ after administration and remained appreciable at $4 \mathrm{~h}$.

Conclusion: Methylone and MDMA produced physiological and euphoric effects typically associated to psychostimulants drugs, with less intense subjective effects in case of methylone. Concentrations of both substances were elevated and in the case of MDMA in the range of those described in experimental studies. Oral fluid could be a useful non-invasive biological matrix of detection after acute methylone use.

The investigation was partially funded by grants from the Instituto de Salud Carlos III (ISCIII, Fondo de Investigación en Salud (FIS)-Fondo Europeo de Desarrollo Regional (FEDER), Grant Numbers: FIS PI17/019629), Predoctoral grants FI18/00179 to Lourdes Poyatos, ISCIII-Red de Trastornos Adictivos (RTA), Grant Number: RD16/0017/0003, AGAUR Gencat Suport Grups de Recerca, Grant Number: 2017 SGR 316 and The European Commission (Predicting Risk of Emerging Drugs with In silico and Clinical Toxicology [PREDICT, Grant number: HOME/2014/JDRU/AG/DRUG/7082]).

\section{9}

Human pharmacology of methylone: a pilot phase I dose-finding study

PhD student Lourdes Poyatos ${ }^{1,2}$, MD, Phd Esther Papaseit ${ }^{1,2}$,

MD, PhD Clara Pérez-Mañă ${ }^{1,2}, \mathrm{NR}$ Soraya Martin ${ }^{1}$, MD Olga Hladun ${ }^{1,2}$, RN Alexandra Vila ${ }^{1}$, DPharm Adrián Siles ${ }^{3}, \mathrm{MD}, \mathrm{PhD}$ Daniel Fuster ${ }^{4}$, $\mathrm{MD}, \mathrm{PhD}$ Ana Maria Barriocanal ${ }^{1,2}, \mathrm{PhD}$ Simona Pichini ${ }^{5}$,

MD, PhD Magí Farré, 1,2

${ }^{1}$ Department of Clinical Pharmacology, Hospital Universitari Germans Trias i Pujol and Institut de Recerca Germans Trias i Pujol (HUGTiPIGTP), Badalona, Spain

${ }^{2}$ Department of Pharmacology, Therapeutics and Toxicology, Universitat Autònoma de Barcelona (UAB), Barcelona, Spain

${ }^{3}$ Department of Pharmacy, Hospital Universitari Germans Trias i Pujol and Institut de Recerca Germans Trias i Pujol (HUGTiP-IGTP), Badalona, Spain

${ }^{4}$ Department of Internal Medicine, Hospital Universitari Germans Trias i Pujol and Institut de Recerca Germans Trias i Pujol (HUGTiP-IGTP), Badalona, Spain

${ }^{5}$ National Centre on Addiction and Doping, Istituto Superiore di Sanità (ISS), Rome, Italy

Introduction: Methylone (3,4-methylenedioxymethcathinone) is one of the most popular synthetic cathinones. It is a beta-keto analogue of the ecstasy (MDMA) with certain pharmacological similarities commonly used as an alternative of classical psychostimulants. Until date, all available information has been provided by epidemiological data from drug forums, surveys to recreational users and cases of attendances and emergency admissions. At present, no experimental controlled data of the acute pharmacological effects of methylone in humans has been performed.
Objectives: This pilot study was designed to obtain preliminary data on the acute effects of methylone in humans, and to determine the selected doses for future investigations.

Methods: The design of the study is double-blind, placebo-controlled, crossover and randomized (lower doses were allocated before the higher dose for safety reasons). The study includes three different cohorts of 3 subjects, the doses of methylone increased after the evaluation of the safety and pharmacological effects. Participants were healthy males with recreational experience in psychostimulants. Each subject participates in one cohort that includes three experimental sessions. Doses of methylone will start at $50 \mathrm{mg}$ until $300 \mathrm{mg}$. In the first cohort they received single oral doses of methylone (50 mg and $100 \mathrm{mg}$ and placebo), the second cohort increased the dose of methylone to $150 \mathrm{mg}$. Study variables included: physiological effects (blood pressure, heart rate, temperature, pupil diameter), Maddox wing device to evaluate sedation, subjective effects (different visual analogue scales, ARCI-49 item short form, VESSPA), and blood and urine samples for pharmacokinetics. The protocol was approved by the local Human Research Ethics Committee.

Results: Preliminary results show that the oral administration of 50, 100 and $150 \mathrm{mg}$ of methylone produced dose-related increases in blood pressure, heart rate and pupil diameter and induced psychostimulant-like and euphoric effects (VAS "intensity", "stimulated", "high" and ARCIMBG). At higher doses, methylone produced slight changes in perceptions but no hallucinogenic effects. No serious adverse reactions were observed.

Conclusion: Methylone at doses between 100-150 mg produced the prototypical psychostimulant effects in a dose-related manner, characterized by an increase of physiological measurements accompanied by noticeable pleasurable and euphoric effects. The effects were similar to those previously observed after the administration of MDMA $100 \mathrm{mg}$ and mephedrone $200 \mathrm{mg}$. This range of doses seems safe and could be administered in future controlled clinical trials to evaluate the comparative abuse potential of methylone.

The investigation was partially funded by grants from the Instituto de Salud Carlos III (ISCIII, Fondo de Investigación en Salud (FIS)-Fondo Europeo de Desarrollo Regional (FEDER), Grant Numbers: FIS PI14/00715 and FIS PI17/01962, Predoctoral grants FI18/00179 to Lourdes Poyatos ISCIII-Red de Trastornos Adictivos RTA, Grant Number: RD16/0017/0003, AGAUR Gencat Suport Grups de Recerca, Grant Number: 2017 SGR 316.

83

Growing need for clinical pharmacology services in a tertiary university hospital in Lithuania Ms Simona Stankeviciute ${ }^{1,2}$, Ms Skaiste Kasciuskeviciute ${ }^{1,2}$, Mr Vaidotas Galaune ${ }^{2}$, Ms Lauryna Aukstikalne ${ }^{1,2}$, Dr Evaldas Padervinskis ${ }^{3}$

${ }^{1}$ Lithuanian University of Health Sciences Institute of Physiology and Pharmacology, Kaunas, Lithuania

${ }^{2}$ Hospital of Lithuanian University of Health Sciences Kauno klinikos, Kaunas, Lithuania

${ }^{3}$ Lithuanian University of Health Sciences, Kaunas, Lithuania

Introduction: Clinical practice of clinical pharmacologist, $\mathrm{MD}$, is rather new in Lithuania. The residency program was established back in 2014, and the first graduates started working at university hospitals in 2018.

The competences of clinical pharmacologist, MD, are described in the Medical Norm, which is a legal document, signed by the Minister of Health. The competences held by the clinical pharmacologist, MD, includes consulting on rational medication use, therapeutic drug monitoring (TDM), pharmacovigilance, personalized pharmacotherapy (according the pharmacogenetic testing, pharmacokinetic risk factors (i.e. renal, liver failure, obesity, others)), drug interactions, and others.

Hospital of Lithuanian University of Health Sciences Kauno klinikos is a tertiary university hospital, the biggest in the country. The annual number 
of outpatient consultations exceeds 1.3 million visits and 94 thousand hospital admissions.

Clinical pharmacology services (CPS) was officially established at the hospital at the end of 2018, and started working at the beginning of 2019.

Objectives: Systematically analyze the frequency and indications for clinical pharmacology consultation (CPC).

Methods:Hospital information system (HIS) was used to systematically analyze the frequency and indications for CPC. Indication for CPC and the pharmacotherapeutic group of CPCs were analyzed. Also, we looked at the frequency of CPC calls throughout the time the (CPS) was officially established at the hospital.

Results: For the time being, there is 1.5 full time equivalent of clinical pharmacologist, MD, providing CPS.

The main indications for CPC are TDM (18\% of all consultations), drug interactions (16\%), drug dose selection (15\%), suspected adverse reactions to medication $(15 \%)$, counseling on medication in case of renal failure $(12 \%)$ and haemodialysis $(10 \%)$, liver damage or hepatic insufficiency $(1 \%)$, and counseling on the safety of medication during pregnancy $(2 \%)$. Most CPC are performed in the intensive care unit (about $30 \%$ of all consultations), in the therapeutic units about $20 \%$, in the surgical units $14 \%$, in the pediatric units $-9 \%$, in the psychiatric units $-5 \%$. Approximately $15 \%$ of all CPC are outpatient.

According to the data of the HIS, there was 200 outpatient CPC in 2020 working $4 \mathrm{~h} /$ week. Other clinics in the country do not currently provide outpatient CPC.

The pharmacotherapeutic groups, that were in the request for $\mathrm{CPC}$, were: antibiotics (51\% of all consultations), cardiovascular drugs (10\%), antifungal drugs $(6 \%)$, antiepileptic drugs $(6 \%)$, other mental systems active drugs $(4 \%)$, anticancer drugs $(4 \%)$, antiviral drugs $(2 \%)$. Polypharmacotherapy was the main issue in approximately $17 \%$ of all consults.

The number of CPC has been steadily increasing (Table 1).

Conclusions: The need for CPS in Lithuania will grow in the upcoming years. The greatest need is for comprehensive counseling on the rational use of antimicrobials, especially for severely ill patients with pharmacological risk factors.

\begin{tabular}{|c|c|c|c|c|c|c|c|c|}
\hline Year & \multicolumn{4}{|c|}{2019} & \multicolumn{4}{|c|}{2020} \\
\hline Quarter & Q1 & Q2 & Q3 & Q4 & Q1 & Q2 & Q3 & Q4 \\
\hline No. CPC & 11 & 49 & 27 & 134 & 168 & 189 & 355 & 395 \\
\hline
\end{tabular}

\section{S1- Pharmacovigilance}

11

Does Rivaroxaban Treatment for Atrial Fibrillation Increase The Risk of Syncope, Dizziness or Falls Compared To Other Direct Oral Anticoagulants?

Dr. Ophir Lavon ${ }^{1,2}$, Dr. Daisy Cohen ${ }^{2}$

${ }^{7}$ Clinical Pharmacology and Toxicology Unit, Carmel Medical Center, Haifa, Israel

${ }^{2}$ Rappaport Faculty of Medicine, Technion-Israel Institute of Technology, Haifa, Israel

Introduction: In clinical studies, rivaroxaban treatment in atrial fibrillation patients was associated with syncope, falls and dizziness without clear relation to bleeding, anemia or stroke. This association was not found with other direct oral anticoagulants (DOACs). Rivaroxaban summary of product characteristics (SPC) includes a warning about the risk of syncope and dizziness, while in other DOACs SPC such warning is absent.
Objective: To evaluate the risk of falls, syncope and dizziness not related to bleeding, anemia or stroke in atrial fibrillation patients treated with DOACs.

Methods: A retrospective observational comparative study of atrial fibrillation adult patients treated with DOACs between January 1, 2013 and December 31, 2017 was performed. Medical records were digitally retrieved from hospital and community computerized databases and extensively surveyed for epidemiological and clinical data. Primary endpoint was either an emergency department (ER) visit or a hospitalization due to syncope, fall or dizziness. Cases related to bleeding, anemia or stroke were excluded. Sampled manual examination of the records validated the data.

Results: Of 6,467 eligible patients, 256 (4\%) were hospitalized or referred to ER due to fall, syncope or dizziness during a mean observation period of 20.1 months. After multivariate regression analysis, statistically significant independent risk factors were found to be older age $(\mathrm{HR}=1.04, \mathrm{p}<0.0001)$ and benzodiazepine use $(\mathrm{HR}=1.33, \mathrm{p}=0.03)$. No statistical difference was found between the different types of DOACs regarding the incidence of ER visits and hospitalization due to syncope, falls and dizziness.

Conclusions: Rivaroxaban treatment in patients with atrial fibrillation was not found to be a significant risk factor for syncope, dizziness, or falls, not related to bleeding, anemia or stroke, and requiring ER referral or hospitalization compared to the other DOACs.

42

Sour is not sour always! Chewable ascorbic acid significantly improves symptoms of radiation therapy induced xerostomia and quality of life

Dr. Samya Dutta ${ }^{1}$, Dr. Sandip Mukhopadhyay ${ }^{1}$,

Prof. Supreeti Biswas ${ }^{2}$, Prof. Sanatan Banerjee ${ }^{3}$, Prof. Abhijit Das ${ }^{1}$

${ }^{1}$ Pharmacology, Burdwan Medical College, Burdwan, India

${ }^{2}$ Pharmacology, NRS Medical College, Kolkata, India

${ }^{3}$ Radiation Oncology, Burdwan Medical College, Burdwan, India

Introduction: Xerostomia or subjective sensation of dryness of mouth is a major cause of morbidity affecting almost $70 \%$ of head and neck cancer patients receiving radiotherapy. Mechanical stimulation of the salivary glands with chewing gums can provide some benefit. Chewable tablets of ascorbic acid may be helpful in such situation due to its citrus or sour test that stimulates salivation as well as by the antioxidant property.

Objectives: To compare the effect of chewable ascorbic acid tablet and mechanical stimulation for the prevention of radiation-induced xerostomia. Methods: Following IEC approval, consenting adult head and neck cancer patients were randomized into ascorbic acid group or chewing gum group and received either $500 \mathrm{mg}$ ascorbic acid chewable tablet twice daily (to be kept in mouth for 5 minutes and then chewed up, if not finished) or sugar free chewing gum (to be chewed for 5 minutes twice daily) before meal for the whole course of radiation. Amount of unstimulated and stimulated whole saliva, percentage change in salivary flow, observer-rated xerostomia score (XS) were noted on day 1, 7, 14, 28 and end of radiotherapy. Quality of life (QoL) were noted on day 1 and at the end of radiation by using EORTC QLQ-H\&N35 questionnaire.

Results: Out of total 60 randomized patients, 54 completed follow up. Though reduction were noted from the baseline, unstimulated and stimulated salivary flow was significantly higher in the ascorbic acid group during all follow up visits after 2 weeks. Mean reduction of salivary flow of $15.33 \%$ (unstimulated) and $11.12 \%$ (stimulated) were noted in the ascorbic acid group compared to $35.88 \%$ (unstimulated) and $18.62 \%$ (stimulated) in the chewing ( $p, 0.001)$ after radiation treatment. Significantly better Observer-rated xerostomia scores (XS) and QoL were noted in ascorbic acid group.

Conclusion: Chewable ascorbic acid could be an inexpensive and simple method in the management of xerostomia and may be explored further. 
Table: Comparison of unstimulated salivary amounts per minute

\begin{tabular}{llll}
\hline & $\begin{array}{c}\text { Chewable ascorbic } \\
\text { acid group } \\
\text { (gram/minute) }\end{array}$ & $\begin{array}{c}\text { Mechanical stimulation } \\
\text { group }^{\#} \\
\text { (gram/minute) }\end{array}$ & $\begin{array}{l}\mathrm{p} \\
\mathrm{v}- \\
\text { al- } \\
\mathrm{ue}\end{array}$ \\
\hline Day 1 & $0.48 \pm 0.03$ & $0.48 \pm 0.03$ & 0.89 \\
Day 7 & $0.46 \pm 0.03$ & $0.45 \pm 0.02$ & 0.11 \\
Day 14 & $0.44 \pm 0.02$ & $0.41 \pm 0.02$ & 0.001 \\
Day 28 & $0.42 \pm 0.02$ & $0.37 \pm 0.02$ & 0.001 \\
$\begin{array}{c}\text { End of } \\
\text { treatment }\end{array}$ & $0.41 \pm 0.02$ & $0.31 \pm 0.03$ & 0.001 \\
\hline
\end{tabular}

*All values in Mean \pm SD

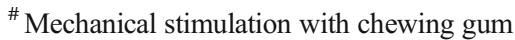

\#\# End of treatment: Mode 42 days

87

Prevalence, Severity and Risk Factors for Prescribing Errors in COVID-19 patients: an analysis of post-hospitalized patients in the Netherlands

Rashudy F Mahomedradja ${ }^{1}$, Dr. Tessa O. van den Beukel ${ }^{2}$, Maaike Bos ${ }^{1}$, Steven Wang ${ }^{1}$, Kirsten Kalverda-Mooij ${ }^{3}$, Dr. Birgit Lissenberg-Witte ${ }^{4}$, Marianne Kuijvenhoven ${ }^{5}$,

Dr. Kim Sigaloff ${ }^{1}$, Dr. Jelle Tichelaar ${ }^{1}$, Prof. dr. Michiel van Agtmael ${ }^{1}$

${ }^{1}$ Department of Internal Medicine, Section Pharmacotherapy, Amsterdam UMC location VUmc, Amsterdam, The Netherlands

${ }^{2}$ Department of Internal medicine and Geriatrics, Amsterdam UMC location VUmc, Amsterdam, the Netherlands

${ }^{3}$ Department of Pulmonology, Amsterdam UMC location VUmc, Amsterdam, the Netherlands

${ }^{4}$ Department of Epidemiology and Biostatistics, Amsterdam UMC location VUmc, Vrije Universiteit Amsterdam, Amsterdam, the Netherlands ${ }^{5}$ Department of Clinical Pharmacology and Pharmacy, Amsterdam, the Netherlands

Introduction: The novel severe acute respiratory syndrome coronavirus 2 (SARS-CoV-2) causing the coronavirus disease 2019 (COVID-19) has challenged healthcare globally. The Netherlands was no exception, leading to a sudden, strong increase of patients in need of hospitalisation or intensive care unit (ICU) treatment. This required reorganisation of inhospital care and health care professionals to participate in acute care, often performing beyond their own medical expertise. From previous studies it is known that a heavy workload, a high turnover of doctors and frequent patient tranfers are associated with prescribing errors (PEs). PEs, on their turn, can lead to adverse drug events (ADEs), resulting in patient harm and hospital (re)admissions. Given these circumstances, it is hypothesized that hospitalized COVID-19 patients are at risk for medication-related harm due to inappropriate prescribing.

Objectives: To determine prevalence, severity and risk factors for PEs in hospitalized COVID-19 patients during the first outbreak of SARS-CoV2 in the Netherlands.

Methods: A single-centre study was performed between 1 March 2020 and 1 October 2020 in Amsterdam UMC - location VU, a 733-bed academic hospital in the Netherlands. A multidisciplinary 'pharmacotherapy team' was compiled to enhance pharmacotherapeutic care at time of postCOVID outpatient clinic (PCOC) visit, approximately 3 months after discharge. The team consisted of a clinical pharmacist, an internist, an hospital pharmacist (all clinical pharmacologists i.t.), a junior medical doctor and a medical student performing medication anamnesis and a structured medication review.
The primary outcome was the number of PEs originated during or after de course of COVID-19 - hospitalisations. Secondary outcomes were the severity of and risk factors contributing to identified PEs.

Results: In total, 102 patients where scheduled for assessment and 98 patients are included in analysis. During COVID-19 - admission and discharge, 65 PEs (55 unintentional drug discrepancies and 10 inappropriate prescriptions) were identified, originated during hospitalisation. After discharge and PCOC visit, an additional 38 PEs were identified. Multivariate analysis shows that an ICU admission and the comorbidity asthma and/or COPD are significantly associated risk factors for PEs introduced during or after the course of hospitalisation.

Conclusion: This is the first study to evaluate the quality and outcome of pharmacotherapeutic care during the SARS-CoV-2 pandemic. To avoid medication-related patient harm in times of unusual hospital capacity and care, we suggest pharmacotherapeutic stewardship by a multidisciplinary team for COVID-19 patients, shortly before discharge.

\section{S2- COVID Vaccines- certainties \& uncertainties}

65

Effects of dalteparin on anti-Xa levels cannot be predicted in critically ill COVID-19 patients

MD, PhD Charlotte Stolk-van der Heijden ${ }^{1}$, MD Emma Kooistra ${ }^{2}$, PhD Rob ter Heine ${ }^{3}$, PhD Elke de Grouw ${ }^{4}, \mathrm{MD}, \mathrm{PhD}$ Tim Frenzel ${ }^{2}$, $\mathrm{MD}, \mathrm{PhD}$ Peter Pickkers ${ }^{2}$, MD, PhD Jenneke Leentjens ${ }^{1}$

${ }^{1}$ Department of Internal Medicine, Radboud University Medical Center, Nijmegen, the Netherlands

${ }^{2}$ Department of Intensive Care Medicine, Radboud University Medical Center, Nijmegen, the Netherlands

${ }^{3}$ Department of Pharmacy, Radboud University Medical Center, Nijmegen, the Netherlands

${ }^{4}$ Department of Clinical Chemistry, Radboud University Medical Center, Nijmegen, the Netherlands

Introduction: Critically ill patients with COVID-19 are at high risk of thromboembolic events despite regular thromboprophylaxis with lowmolecular weight heparins (LMWHs), while intensifying thromboprophylaxis is associated with bleeding. This raises the question whether pharmacokinetic (PK) and pharmacodynamic (PD) properties of LMWHs are predictable in these patients.

Objectives: To characterize the PK and PD properties of dalteparin in critically ill COVID-19 using PK/PD modelling with anti-Xa as readout and to investigate whether current standard dosing regimens are expected to result in anti-Xa levels within target ranges.

Methods: We prospectively included 15 adult patients who were admitted to the intensive care unit of the Radboudumc, Nijmegen, the Netherlands with COVID-19 (age range 32-76 years, weight 70$110 \mathrm{~kg}$, endogenous creatine clearance $16-214 \mathrm{~mL} / \mathrm{min}$ ). All received dalteparin in prophylactic- or intermediate-intensity (5000 IU once daily (OD) for those $<100 \mathrm{~kg}, 5000$ IU bidaily (BD) for those $\geq 100$ $\mathrm{kg}$ ) or therapeutic-intensity (100 IU/kg BD). We collected 102 anti$\mathrm{Xa}$ samples on regular timepoints, on one to three days per patient. PK analysis of dalteparin was performed by nonlinear mixed-effect modeling using NONMEM version 7.4. Rate constants, volume and clearance parameters were allometrically scaled to a total body weight of $70 \mathrm{~kg}$. Various easy to obtain clinical parameters, such as vasopressor use and edema scores for absorption rate and glomerular filtration rate (estimated with the Chronic Kidney Disease Epidemiology Collaboration (CKD-EPI) algorithm and endogenous creatinine clearance based on urine collection) for elimination rate were tested as covariates in the model. Subsequently, we used the final model to perform Monte Carlo simulations to predict sub- or supraoptimal dalteparin treatment with standard dosing.

Results: The data were well-fitted to a linear one compartment model with first order absorption and elimination. The volume of distribution of 
dalteparin was estimated at 14.6L (residual standard error (RSE) 14\%), elimination rate at $918 \mathrm{~mL} / \mathrm{h}$ (RSE 19\%). A wide interindividual variation in absorption (78\% (RSE 56\%)) and clearance rate (34\% (RSE $87 \%)$ ) of dalteparin was observed, unexplained by any of the tested clinical covariates. We simulated that current dosing regimens frequently result in offtarget anti-Xa levels. Most strikingly, dalteparin 5000 IU BD was expected to result in anti-Xa levels over a prophylactic range of $0.2-0.4 \mathrm{IU} / \mathrm{ml}$ in $46 \%$ of patients. Therapeutic-intensity dalteparin was expected to result in anti-Xa levels over this range in $97 \%$ of cases, and under and over a therapeutic range of $0.6-1.0 \mathrm{IU} / \mathrm{ml}$ in $22 \%$ and $19 \%$ of patients, respectively.

Conclusions: Anti-Xa levels during dalteparin treatment are difficult to predict in critically ill COVID-19 patients, due to a wide unexplained interindividual variation in absorption and clearance of this LMWH. Most importantly, we expect that current dosing regimens frequently result in off-target anti-Xa levels in these patients. Until randomized controlled trials advise on the safest dosing, this argues for anti-Xa measurements to reach advised target ranges in secondary thromboprophylaxis. For primary thromboprophylaxis, the clinician should be aware that intermediate- or therapeutic-intensity dalteparin often result in anti-Xa levels well over standard prophylactic targets.

71

Longitudinal analysis of COVID-19 infection rates and antibody levels pre- and post- vaccination

Dr Jorg Taubel ${ }^{1}$, Dr Samuel Thomas Cole ${ }^{1}$, Dr Christopher S Spencer ${ }^{2}$, Ms Anne Freier $^{2}$, Dr Isobel Atkin ${ }^{2}$, Dr Ibon Garitaonandia ${ }^{2}$, Dr Ulrike Lorch

${ }^{1}$ Richmond Research Institute, London, United Kingdom

${ }^{2}$ Richmond Pharmacology Ltd, London, United Kingdom

Introduction: By January 2020, the novel coronavirus (SARS-CoV-2) had been detected in Wuhan, China and identified as the causative agent for COVID-19. By March 2020, it had become a global pandemic, prompting diverse public health strategies around the world, including increased hygiene, face mask mandates, and local and national lockdowns. In December 2020, a COVID vaccination program started in the UK, and continues to rapidly expand. Concurrently, multiple strains of SARS-CoV-2 have been detected in the UK, with differing transmission rates and levels of responsiveness to licensed vaccines.

Objectives: The primary objective of this project is to use antibody tests to examine the extent to which infection and vaccination provide immunity. Alongside this, we are using genetic screening methods to establish whether any reinfections are the result of novel strains of SARS-CoV-2. Methods: Our study cohorts consisted of staff and trial participants at a Clinical Research Organisation in London. We are using a chemiluminescent microparticle immunoassay (CMIA) and an electrochemiluminescence immunoassay (ECLIA) test to quantify antibodies in individuals with PCR-confirmed SARS-CoV-2 infection and individuals receiving a vaccine. Genetic screening in individuals with confirmed reinfection is being performed to determine the strain with which they have been infected.

Results: Preliminary data was assessed using a population pharmacokinetic analysis (popPK) in the form of a generalised estimating equation model and established that natural SARS-CoV-2 infection generated an antibody response with a half-life of 85.3 days. Our results agree with those of other published studies using alternative biochemical methods to quantify SARS-CoV-2-specific antibody titres, to demonstrate waning antibody titres over the course of 90 days post-infection. In the coming weeks, we will be able to compare these results with the antibody responses generated by a COVID-19 vaccine.

We found at least one case of an individual being reinfected with SARSCoV-2. Genetic screening is being carried out to establish whether the second infection is a different strain of SARS-CoV-2.
Conclusion: The use of a generalised estimating equation allows us to closely relate IgG levels with longer term outcomes. As this is an ongoing research project, our sample size continues to grow, and our model will continue to increase in accuracy. As more individuals in the UK receive vaccines, we will be able to quantitatively compare the quality and duration of the antibody response from natural infection and from vaccination. We will also be able to compare symptomologies in the case of reinfection with initial infection to establish the extent of the protectivity of COVID vaccines.

\section{Poster Session 2a - PK-PD-DDI-PhG}

45

Variability in the antioxidant MSRA gene affects personality dimensions and psychopathology of patients with anorexia nervosa Miss Luz M González ${ }^{1}$, Mrs Angustias García-Herráiz ${ }^{2}$, Miss Sonia Mota-Zamorano ${ }^{1}$, Mr David Albuquerque ${ }^{3}$,

Mrs Isalud Flores ${ }^{2}$, Mr Guillermo Gervasini ${ }^{1}$

${ }^{1}$ Dpt. Medical \& Surgical Therapeutics, Medical School, University of Extremadura, Badajoz, Spain

${ }^{2}$ Eating Disorders Unit, Institute of Mental Disorders, Extremadura Health Service, Badajoz, Spain

${ }^{3}$ Genomics Group, Fundación Investigación Hospital General Universitario de Valencia, Valencia, Spain

Introduction: Anorexia Nervosa (AN) is a complex disorder characterized by severe food restriction, fear of weight gain and body image distortion, all of which leads to the highest mortality rate of any psychiatric disease (Arcelus et al., 2011). Traditionally, socio-cultural and family factors have been considered the most important contributors to AN; however, in the last years, twin and family studies have demonstrated the existence of high heritability estimates (Bulik et al., 2006, Thornton et al., 2011), thus pointing out genetics as a key player in this eating disorder (ED) (Pinheiro et al., 2009). The methionine sulfoxide reductase A (MSRA) gene encodes an enzyme (MsrA) that reduces oxidized methionine residues, contributing significantly to the defense against oxidative stress (Kim and Gladyshev, 2007). This gene is highly expressed in several areas of the brain and has in fact been related to several psychiatric conditions such as schizophrenia (Walss-Bass et al., 2009), bipolar disorder (Ni et al., 2015) or Alzheimer's (Gabbita et al., 1999) and Parkinson's disease (Wassef et al., 2007), as well as being associated with a variety of behavioral traits and psychiatric symptoms (Reiterer et al., 2019). The presence of genetic variants in the MSRA gene has also been linked to obesity. Indeed, three meta-analyses of GWAS have suggested that certain loci in or near this gene are body-mass index (BMI) regulators (Lindgren et al., 2009, Scherag et al., 2010, Dorajoo et al., 2012), which has been confirmed in some (Bille et al., 2011, Albuquerque et al., 2014, Krishnan et al., 2017) but not all (Hotta et al., 2010, Gonzalez et al., 2014, Volckmar et al., 2016) follow-up studies.

Objective: To determine whether variability in the MSRA gene, related to obesity and several psychiatric conditions, may be relevant for traits and psychopathological symptoms common in Anorexia Nervosa (AN) and/ or for the susceptibility to the disorder.

Methods: A total of 629 women (233 AN patients and 396 controls) were genotyped for 14 tag-SNPs. Psychometric evaluation was performed with the EDI-2 and SCL-90R questionnaires. Genetic associations were carried out by logistic regression controlling for age.

Results: Two tag-SNPs, rs11249969 and rs81442, were associated with the global EDI-2 score, which measures EDI-related personality dimensions (adjusted FDR-q=0.02 and 0.04, respectively). Moreover, rs81442 significantly modulated all the scales of the SCL-90R test that evaluates general psychopathology (FDR-q values ranged from 4.1E-04 to 0.011). A slidingwindow analysis using adjacent 3-SNP haplotypes revealed a proximal region of the MSRA gene spanning $187.8 \mathrm{Kbp}$ whose variability deeply affected traits and psychopathological symptoms of the AN patients. Depression was the item that showed the strongest association with any of 
the constructed haplotypes (FDR-q=3.60E-06). No variants were found to be linked to AN risk or anthropometric parameters in patients or controls. Conclusions: Variability in the MSRA gene locus modulates personality traits and psychopathological symptoms shown by AN patients.

51

The use of genotyping TPMT polymorphisms to reduce the risk of adverse reactions in patients treated with thiopurine drugs Miss Ana Casajús Rey ${ }^{1}$, Miss Diana María Campodónico, Miss Marta Méndez Rodríguez, Miss Antía Gómez Fernández, Miss Gina Paola Mejía Abril, Mrs. Dolores Ochoa Mazarro, Miss Paola Camargo Mamani, Mister Francisco Abad-Santos ${ }^{1}$ Hospital Universitario De La Princesa, Servicio De Farmacología Clínica, Madrid, Spain

Introduction: Thiopurine methyltransferase (TPMT) is an enzyme that metabolizes a class of drugs known as thriopurines among which we can find azathioprine or mercaptopurine. The use of these drugs has increased in the past years because they are being used to treat different immunerelated conditions and blood disorders such as leukemia. Different polymorphisms of this enzyme have been described among which we can find those that lead to an inactivation and therefore to a lower degradation and greater predisposition to suffer adverse reactions such leukopenia.

Objectives: The overall objective is to demonstrate the use of genotyping TPMT polymorphisms prior to initiating treatment with thiopurines to reduce the risk of adverse reactions in patients with the mutation by adjusting the dose.

Materials and Methods: Our project consists of an observational and retrospective study approved by CEIm of Hospital Universitario de La Princesa (HUP). Patients that meet all the inclusion criteria (all patients were over 18 years old whose follow up has been done at our hospital and were TPMT genotyped before June of 2019 and starting treatment with thiopurines) were selected to be followed for six months from the start of the treatment and it was checked whether a toxicity monitoring had been done in accordance to recommendations.

Data was collected from a local database located on the HUP internal network. The database of this study was codified to respect the confidentiality of every patient.

Results: There has been 644 genotyped patients until June of 2019 at Hospital Universitario La Princesa in Madrid of which 440 patients were followed at this Hospital to analyze and determine their allelic distribution. There were $6.59 \%$ carriers of mutated polymorphisms $(6.14 \%$ heterozygous and $0.45 \%$ homozygous mutated); the predominant allelic variant was TPMT*3A $(4.55 \%)$.

Preliminary results have been obtained with medical records of 131 patients, from these; only 47 patients met all the inclusion criteria. In this sample, dose recommendations are only applied in mutated subjects, receiving a lower dose than wild-type subjects. The recommendations for close monitoring of the patients were not followed in most patients. The incidence of adverse reactions was $42,55 \%$ with no significant difference between wild-type $(39,47 \%)$ and mutated $(55,56 \%)$. The incidence of leukopenia was $4,26 \%$ with no difference between wild-type and mutated. The study is being continued with the remaining patients, expecting to obtain similar or even better results.

Conclusion: Genotyping TPMT before starting treatment with thiopurines would allow us to know the activity of the enzyme and classify those who suffer inactivation of the same, thus being able to reduce the dose of the drug and therefore the adverse reactions in these patients.

\section{5}

Pharmacokinetics and acute effects of a cannabis standardized preparation after oral and vaporized administration MD Ana Pilar Pérez-Acevedo ${ }^{1,2}, \mathrm{PhD}$ student Lourdes Poyatos ${ }^{1,2}$, $\overline{\mathrm{MD}, \mathrm{PhD} \text { Esther Papaseit }}{ }^{1,2}, \mathrm{MD}, \mathrm{PhD}$ Clara Pérez-Mañá ${ }^{1,2}$,
RN Soraya Martin ${ }^{1,2}$, MD Olga Hladun ${ }^{1,2}$, MD, PhD Magin Farre Albaladejo $^{1,2}, \mathrm{PhD}$ Simona Pichini ${ }^{3}, \mathrm{PhD}$ Francesco Paolo Busardó ${ }^{4}$, PhD Roberta Pacifici ${ }^{3}$, PhD Giulio Mannocchi ${ }^{5}$, PhD Massimo Gottardi $^{6}$, PhD AnnaGiulia Di Trana ${ }^{4}$

${ }^{1}$ Clinical Pharmacology Unit, Hospital Universitari Germans Trias i Pujol and Institut de Recerca Germans Trias i Pujol (HUGTiP-IGTP), 08916 Badalona, Spain,

${ }^{2}$ Department of Pharmacology, Therapeutics and Toxicology, Universitat Autònoma de Barcelona, 08193 Cerdanyola del Vallés, Spain

${ }^{3}$ National Centre on Addiction and Doping, Istituto Superiore di Sanità, Rome, Italy

${ }^{4}$ Department of Excellence-Biomedical Sciences and Public Health, Università Politecnica delle Marche, Ancona, Italy

${ }^{5}$ School University of Camerino, Camerino, Italy

${ }^{6}$ Comedical srl, Trento, Italy

Introduction: The use of standardized preparations with cannabis flower cups for therapeutic/medical purposes is increasing, especially after oral administration of decoction and oil preparations. There are very few studies evaluating the pharmacological effects and pharmacokinetic of the same medicinal cannabis preparations administered by different routes in humans.

Objectives: To know the concentrations of cannabinoids and their metabolites in biological matrices (serum, oral fluid, sweat, urine) and acute pharmacological effects after the administration of a standardized cannabis preparation in oral formulation (decoction, oil) and vaporized.

Methods: The study design was open-label, non-randomized, singleblind with three different formulation (oral decoction, oral oil, vaporized). Only one formulation was administered per session. The standardized cannabis product was FM2 from Italy that contains a $6 \%$ of tetrahydrocannabinol (THC) and a $8 \%$ of cannabidiol (CBD). A total of 43 young healthy volunteers with recreational users of cannabis participated in the study ( 37 males and 6 females). Thirteen subjects were treated with 100 $\mathrm{mL}$ of cannabis decoction (1.8 $\mathrm{mg}$ of THC and $2.7 \mathrm{mg}$ of CBD), other thirteen with $0.45 \mathrm{ml}$ of oil ( $1.8 \mathrm{mg}$ of THC and $3.8 \mathrm{mg}$ of CBD) and fourteen inhaled $100 \mathrm{mg}$ of cannabis ( 0.6 to $2 \mathrm{mg}$ and CBD 0.8 to $3 \mathrm{mg}$ ) using the Volcano vaporizer. Serial blood samples were obtained along 24 hours. In addition oral fluid and urine were collected. Pharmacological effects were evaluated using standardized questionnaires and vital signs determination. Concentrations of cannabinoids in different matrices were quantified with validated methods.

Results: For the oral route, although oil contained significantly more THC, blood concentrations of 11-OH-THC, THC-CCOOH, and THC$\mathrm{COOH}$ Gluc were similar in both oral formulations (oil and decoction). THC was absorbed similarly in decoction and oil. On the contrary, although the oil contained significantly more CBD, the absorption was significantly higher with the decoction. After inhalation, concentrations of THC and CBD were very higher in comparison to oral route, with faster peak concentration at $10 \mathrm{~min}$ in comparison to oral (1.3-1.8 hours). The THC-COOH Gluc was detected mainly in urine samples, followed by THC-COOH and THC-Gluc, and the most excreted CBD metabolites were 7-OH-CBD, and 7-COOH-CBD in the three formulations.

The decoction induced a greater sensation of hunger and drowsiness than oil, but not euphoria-related effect were observed. The vaporization produced the typical effects of THC with feeling of high, intensity, good effects,hunger, drowsiness, and very mild changes in blood pressure and a significant increase in heart rate. No serious adverse effects were observed.

Conclusions: Pharmacokinetic of cannabinoids, their precursors and their metabolites in biological fluids from individuals treated with oral and vaporized medicinal cannabis (decoction, oil, vaporization) cannabis showed high inter-individual variability, in spite that we use the same standardized preparation. This variability is probability due to the difficulties in standardization the exact dose of cannabis compounds (THC/CBD) when administered by different preparations and routes. 
Funding: Instituto de Salud Carlos III (ISCIII-FEDER), FIS PI14/00715 and PI20/00879, Red Trastornos Adictivos RD16/0017/0003, Plataforma Unidades Investigación y Ensayos Clínicos-SCREN, PT 17/0017/0016; Ministerio de Sanidad PNSD 2015 I054.

\section{1}

Experience of implementation of Fluoropyrimidines treatment individualized recommendations based on DPYD genotype in a Clinical Pharmacology Department

Elena Diago Sempere ${ }^{1}$, Antonio Caballero Bermejo ${ }^{1}$, Almudena Ramirez García ${ }^{1}$, Gustavo Centeno Soto ${ }^{1}$, Concepción Payares Herrera ${ }^{1}$, Laura Javaloyes Garachana ${ }^{1}$, Belén Ruiz-Antorán ${ }^{1}$, Aranzazu Sancho López ${ }^{1}$, Cristina Avendaño Solá ${ }^{1}$ ${ }^{1}$ Hospital U Puerta de Hierro, Majadahonda, Madrid, Spain

Introduction: Fluoropyrimidines as 5-FU or capecitabine are frequently prescribed anticancer drugs and part of the treatment of most gastrointestinal tumors. They are inactivated in the liver by the dihydropyrimidine dehydrogenase (DPD). Between 3-8\% of the caucasian population have a partial DPD deficiency, and between 0,01 and $0,5 \%$ a complete deficiency. These patients show a significantly increased risk of severe toxicity when treated with regular doses.

In 2020, all EU fluoropyrimidines SPCs were amended to include the recommendation of genotyping in section 4.4, Precautions for use.

Objectives: Our goal was to present the experience of a Clinical Pharmacology (CP) Department at a tertiary hospital implementing a parallel lab and CP pharmacogenetic advice for treatment with FP.

Methods: After inclusion of the genotyping recommendation in SPCs, a simple process was created to allow the oncologist to perform, in a single action, a request to Molecular Genetics Laboratory and a medical consultation to our CP Department. CP interprets the result issued by the lab and provides a recommendation for fluoropyrimidines dosage with advice on overall patient's pharmacological treatment.

We conducted a retrospective analysis of all cases since the implementation by June 2020 .

Results: A total of 125 DPYD genotyping determinations were requested. Only 9 out of $125(7,3 \%)$ patients presented any DPYD decreased activity variants; 8 out of these 9 patients started treatment, 5 with initial dose adjustments, and 3 patients required additional dose adjustments during the course of treatment.

109 out of 125 (67\%) patients started treatment once DPYD CP recommendations were available. This is interpreted as a signal of acceptability of the process, as it implies a change in behaviour of treating physicians, with early identification of candidates to 5-FU treatment, in order to have results in a timeline manner.

Conclusion: Recommendations by $\mathrm{CP}$ for individualised fluoropyrimidines treatment is valued by treating physicians even though the number of advices recommending a change in treatment was low.

The involvement of $\mathrm{CP}$ providing interpretation and overall treatment recommendations beyond the actual lab DPYD genotyping, can be easily implemented in clinical practice through multidisciplinary collaboration.

\section{2}

\section{Evaluation of body surface area adjusted dosing of high-dose} methotrexate by population pharmacokinetics in a large cohort of cancer patients

Dr. rer. nat. Usman Arshad $^{1,3}$, Dr. med. Max Taubert ${ }^{1}$, Dr. med. Tamina Seeger-Nukpezah $^{2}$, Dr. rer. nat. Sami Ullah ${ }^{1,3}$, Dr. rer. nat. Kirsten C. Spindeldreier ${ }^{4}$, Dr. rer. nat. Ulrich Jaehde ${ }^{3}$, Dr. med. Michael Hallek ${ }^{2}$, Dr. med. Uwe Fuhr ${ }^{1}$, Dr. med. Jörg Janne Vehreschild ${ }^{2,5,6}$ M. Sc. Carolin Jakob ${ }^{2}$

${ }^{1}$ University of Cologne, Faculty of Medicine and University Hospital Cologne, Department I of Pharmacology, Cologne, Germany
${ }^{2}$ University of Cologne, Faculty of Medicine and University Hospital Cologne, Department I of Internal Medicine, Cologne, Germany

${ }^{3}$ Institute of Pharmacy, Clinical Pharmacy, University of Bonn, Bonn, Germany

${ }^{4}$ University Hospital Cologne, Hospital Pharmacy, Cologne, Germany

${ }^{5}$ German Center for Infection Research (DZIF), partner site BonnCologne, Cologne, Germany

${ }^{6}$ Goethe University Frankfurt, Faculty of Medicine and University Hospital of Frankfurt, Department of Internal Medicine, Hematology and Oncology, Frankfurt am Main, Germany

Introduction: Methotrexate (MTX) is an effective drug for the treatment of various malignant disorders. Dose individualization is challenging due to high between-subject variability (BSV) of its pharmacokinetics (PK) and toxicity, necessitating therapeutic drug monitoring (TDM). Dose optimization using PK covariates may improve initial attainment of target concentrations. Typically, dosing of MTX is linearly scaled according to patient body surface area (BSA), but this approach faces concerns due to potential under/over-exposure, also considering the arbitrary nature of BSA estimates attributed to the variety of equations used. Sex may be another covariate as sex dependent regulation of renal transporters with a possible role in MTX elimination was recently reported.

Objectives: The primary objective was to elucidate covariates associated with MTX exposure, including patient sex and BSA. The study further aimed to assess the current clinical practice of dosing MTX based on BSA.

Methods: A retrospective analysis of MTX concentrations and covariate data from patients with malignancies treated at the University Hospital of Cologne between 2005 and 2018 was conducted using population PK by nonlinear mixed effect modeling. Patient demographics and clinical chemistry data were examined as covariates for PK parameters. Probability of target attainment (PTA) under BSA based, flat and stratified dosing regimens was computed by simulating virtual patients to compare plasma drug exposure for dose levels of 500 to $4000 \mathrm{mg} / \mathrm{m}^{2}$ BSA (reference dose). Combined target criteria were that plasma concentrations should not exceed 1.0 and $0.3 \mathrm{mg} / \mathrm{L}$ at $42 \mathrm{~h}$ and $48 \mathrm{~h}$ after start of infusion, and that the achieved AUC should be within $\pm 30 \%$ of the AUC of a subject with a BSA of $1.73 \mathrm{~m}^{2}$

Results: Plasma concentration measurements (2,182 observations) from TDM from 229 cancer patients were used for the analysis. A three-compartment model with linear elimination best described MTX PK. Clearance (CL) of 4.33 [95\% CI, 2.95-5.92] L h-1 and central volume of distribution of 4.29 [1.81-7.33] L were estimated. BSV of $29.7 \%$ (coefficient of variation) and an inter-occasion variability of $23.1 \%$ were attributed to CL. A 16 [7 - 25] \% lower CL was estimated in female subjects. Serum creatinine, patient age, sex and BSA were significantly related to CL of MTX. Marginal differences in PTA between flat and BSA-based dosing were observed, whereas the stratified dosing performed best overall. Proposed stratified doses (with highest PTA) as an alternative to flat or BSA based doses are presented in the Table.

Conclusion: Stratified dosing across BSA quartiles is suggested to optimize the attainment of target plasma exposure of MTX.

Table: Stratified doses with highest probability of target attainment compared to those based on body surface area and flat doses of MTX administered as $24 \mathrm{~h}$ continuous infusion.

\begin{tabular}{|c|c|c|c|c|}
\hline \multirow[b]{2}{*}{ BSA-based } & \multirow{2}{*}{ Flat } & \multicolumn{3}{|c|}{ Stratified } \\
\hline & & $\begin{array}{c}<25 \% \\
\left(<1.7 \mathbf{m}^{2}\right)\end{array}$ & $\begin{array}{c}25-75 \% \\
\left(1.7-2.12 \mathrm{~m}^{2}\right)\end{array}$ & $\begin{array}{c}>75 \% \\
\left(>2.12 \mathrm{~m}^{2}\right)\end{array}$ \\
\hline $500 \mathrm{mg} / \mathrm{m}^{2}$ & $850 \mathrm{mg}$ & $775 \mathrm{mg}$ & $850 \mathrm{mg}$ & $975 \mathrm{mg}$ \\
\hline $1000 \mathrm{mg} / \mathrm{m}^{2}$ & $1700 \mathrm{mg}$ & $1550 \mathrm{mg}$ & $1700 \mathrm{mg}$ & $1900 \mathrm{mg}$ \\
\hline $2000 \mathrm{mg} / \mathrm{m}^{2}$ & $3400 \mathrm{mg}$ & $3000 \mathrm{mg}$ & $3400 \mathrm{mg}$ & $3800 \mathrm{mg}$ \\
\hline $4000 \mathrm{mg} / \mathrm{m}^{2}$ & $7000 \mathrm{mg}$ & $6400 \mathrm{mg}$ & $7200 \mathrm{mg}$ & $8000 \mathrm{mg}$ \\
\hline
\end{tabular}


82

Pharmacokinetics of temozolomide in cerebrospinal fluid of children with malignant tumors - a pilot study

Sören Büsker ${ }^{4}$, Assoc. Prof. Dr Andreas Peyrl ${ }^{1}$, MD Valentin al Jalali ${ }^{2}$, ao. Univ.-Prof. Mag. Dr. Walter Jäger ${ }^{3}$, Dr. Stefan Poschner ${ }^{3}$,

Dr. Sami Ullah ${ }^{4}$, MD Lobna El Tabei ${ }^{4}$, Prof. Dr. Uwe Fuhr ${ }^{4}$

${ }^{1}$ Department of Pediatrics, Medical University of Vienna, Vienna, Austria

${ }^{2}$ Department of Clinical Pharmacology, Medical University of Vienna, Vienna, Austria

${ }^{3}$ Department of Pharmaceutical Chemistry, Division of Clinical Pharmacy and Diagnostics, University of Vienna, Vienna, Austria

${ }^{4}$ University of Cologne, Faculty of Medicine and University Hospital Cologne, Department I of Pharmacology, Cologne, Germany

Introduction: Temozolomide is frequently used in the treatment of different childhood brain tumors. However, information on its pharmacokinetic profile in cerebrospinal fluid (CSF), which is commonly used as a surrogate for central nervous system exposure, is very limited in children. Objectives: To evaluate whether measurable concentrations of temozolomide are reached and maintained in CSF at steady state after oral administration of temozolomide in children with a recurrent malignant brain tumor and leptomeningeal dissemination or risk of leptomeningeal dissemination.

Methods: Temozolomide was administered at an ongoing schedule with daily oral administrations of $35-195 \mathrm{mg} / \mathrm{m}^{2}$. CSF (5ml) and blood samples $(5 \mathrm{ml})$ were collected during routine examinations in thirteen pediatric patients (age 3 to 19 years). Quantitation of temozolomide in plasma and CSF was performed by reversed-phase high-performance liquid chromatography. Pharmacokinetic evaluation was carried out using non-linear mixed effects modeling (NONMEM software).

Results: In total, 29 plasma samples and 79 CSF samples were used to develop the population PK model. A catenary four-compartment model (depot [1], central/plasma [2], CSF [3] and peripheral compartment [4]), first-order absorption and transfer rates adequately described the data. The absorption rate constant $(\mathrm{Ka})$ was fixed to $2.4 \mathrm{~h}^{-}{ }^{1}$, based on literature. The volume of distribution in the CSF (Vcsf) was fixed to $80 \mathrm{ml}$, which is the mean of age-expected volumes $(4 \mathrm{~mL} / \mathrm{kg}$ in infants weighing $15 \mathrm{~kg}$ or less, $3 \mathrm{~mL} / \mathrm{kg}$ in children, and $2 \mathrm{~mL} / \mathrm{kg}$ in adolescents) in our thirteen pediatric patients.

Temozolomide clearance (CL) from the central compartment was 3.31 L/h (95\% CI: 2.19-5.10, CV: $31.6 \%)$, volume of distribution in the plasma (Vc) was 11.0 L (7.10-21.82, CV: $42.5 \%)$ and volume of distribution in the peripheral compartment was $41.8 \mathrm{~L}$. Transfer rates from plasma to CSF (K23) were $0.0079 \mathrm{~h}^{-1}$ and $1.09 \mathrm{~h}^{-1}$ backwards (K32), respectively.

Based on simulations, median AUC [95\% prediction interval] was 17.5 [5.08-42.0] and 8.24 [2.29-22.9] $\mathrm{mg} * \mathrm{~h} / \mathrm{L}$ in plasma and CSF, respectively. The AUCcsf / AUCplasma ratio was 48[27-69]\%.

Conclusion: Measurable concentrations of temozolomide were reached and maintained in CSF at steady state after oral administration of temozolomide. CSF penetration appears to be favorable in children.

98

Sublingual versus Oral Administration of Tacrolimus in Adult Lung Transplant Recipients: A Retrospective

Dr. Iván Mazón Maraña ${ }^{1}$, Dr. Diana Zulema Cuellar Gómez ${ }^{1}$, Dr. María de Mar García Saiz ${ }^{1}$, Dr Beatriz Alonso Gómez ${ }^{1}$ Dr. Rita Nogueiras Álvarez ${ }^{1}$, Dr. David Valencia López ${ }^{1}$,

Dr. Andrea Bautista Blázquez ${ }^{1}$, Dr Victor Manuel Mora Cuesta ${ }^{2}$,

Dr. Blanca Sánchez Santiago ${ }^{1}$, Dr. Mํ'ngeles Lines de $\operatorname{Cos}^{1}$,

Dr Noelia Vega Gil ${ }^{1}$

${ }^{1}$ Servicio De Farmacología Clínica, Hospital Marqués de Valdecilla, Santander, Spain
${ }^{2}$ Servicio De Neuomología, Hospital Marqués de Valdecilla, Santander, Spain

Introduction: Tacrolimus (TC) is a calcineurin inhibitor used as an immunosuppressant in solid organ transplantation. It exhibits great pharmacokinetic variability and is generally administered orally (PO). Sublingual administration (SL) has been postulated as safe and capable of reaching levels in range $(5-15 \mu \mathrm{g} / \mathrm{L})$ in situations in which PO is not possible.

Objectives: To assess the variations in TC monitoring when modifying the SL route and to try to estimate an adequate dosage adjustment for its use when necessary.

Methods: Using the TC monitoring records of our hospital between 2017 and 2020, a review of the episodes with SL use and their comparison with the PO levels achieved in the same patient was made. A total of 8 patients were registered ( 7 men, 1 woman), with lung transplants due to various pathologies, aged between 36 and 63 years and who had presented a total of 12 episodes of change of dosage to SL. The variables evaluated of each episode were: dose, level, weight and concentration/dose ratio (CDR) at 5 time points chosen before, during and after the change in dosage.

Results: 7 of the 8 patients had digestive absorption problems. In the oral dose prior to SL, 5 of 12 cases were within the appropriate range, with 3 being above and 5 below the range. After switching to SL, 6 cases entered the range in a mean of 4 days $( \pm 1.19)$ and 6 reached toxicity levels. This plasma level increase, reaching a toxicity range in some cases, could have contributed to the use of concomitant medication or to specific circumstances at the time of administration (the start of therapy after surgery). In 4 of the 6 cases, the increase in levels could not be justified by the individual circumstances of the patient, so it could only be explained by the change in the presentation of the drug. Comparing for all cases the median CDR of each chronological moment, there was a 56\% increase when switching to SL ( $1.41 \pm 6.25$ for previous PO and $2.53 \pm 2.81$ for 1st determination of $\mathrm{SL})$. The increase was even greater $(217 \%)$ when reaching levels in range $(3.065 \pm 10.19)$ in SL. The decrease when returning to $\mathrm{PO}$ was equivalent to the previous increase $(1.4 \pm 1.126$ for post $\mathrm{PO})$, and was maintained in the subsequent $\mathrm{PO}$ determination $(1.425 \pm 1.26)$.

Conclusion: The increase in CDR when going from PO to SL shows the greater bioavailability of TC when administered via SL because it is not subject to intestinal metabolism. It could be an efficient alternative to PO. This is reinforced by the decreasing CDR when patients switch back to PO. However, we must be cautious when making the switch of presentation, since when the CDR increases there is also a greater risk of reaching plasma levels close to toxicity. Although the data are limited by the small number of cases, we can conclude that SL could be a plausible option in patients with digestive issues or circumstances that do not allow adequate therapeutic compliance.

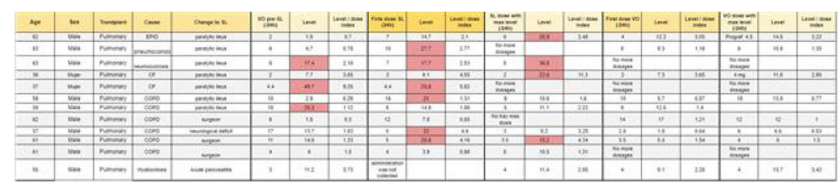

Poster Session 2b - Antimicrobials

30

Outcomes of Penicillin Hypersensitivity Testing in Clinical

Pharmacology Outpatient Clinic

MD, PhD, Assistant Professor Suzana Mimica Matanović ${ }^{1,2}$,

Dr Anja Ivaniš ${ }^{3}$

${ }^{1}$ Faculty of Medicine, Josip Juraj Strossmayer University of Osijek , Osijek, Hrvatska

${ }^{2}$ Unit for Clinical Pharmacology, Department of Internal Medicine, University hospital centre Osijek, Osijek, Croatia

${ }^{3}$ Department of Pediatrics, General Hospital Našice, Našice, Croatia 
Introduction: Penicillins are widely used antibiotics and signficant number of patients have penicillin hypersensitivity in their drug history. It can verified using in vitro and in vivo methods, with skin tests and oral challenge being the most important. Penicillin hypersensitivity could sometimes wane with time

Objectives: The aim of the study was to evaluate the relationship between the results of penicillin hypersensitivity testing and the demographic characteristics of the subjects, time interval between the primary reaction to penicillin and the moment of testing, type and clinical manifestations of primary reaction to penicillin, the existence of hypersensitivity to other allergens and family history of penicillin hypersensitivity.

Methods: In this cross-sectional study data were collected from medical records October 1, 2016 - December 31, 2019 in Clinical Pharmacology Outpatient Clinic, Clinical Hospital Center in Osijek, Croatia. The data included demographic characteristics, results of in vitro penicillin hypersensitivity testing, results of in vivo penicillin hypersensitivity testing (commercial PPL, MDM, diluted ampicillin with positive and negative controls) and detailed characterization of the previous hypersentivity reaction.

Results: Out of 50 subjects, in 49 (98\%) skin prick test was performed and was negative; among them intradermal test was positive in $17(35 \%)$ cases. The rest of the subjects went through oral provocation with amoxicillin, which was positive in only one of them (overall, positive testing results in $36.7 \%$ subjects). Specific IgEs to penicillins were done in 38 (76 $\%)$ subjects and were negative in all of them. In their drug history 23 patients $(48 \%)$ had a reaction within 5 years before testing. The most often used penicillins were amoxicillin or amoxicillin/clavulanic acid. The most common clinical manifestation of penicillin hypersensitivity was maculopapular rash in $26(54 \%)$ subjects. Angioedema in drug history was developed in $5(10 \%)$ subjects and was positively correlated with a positive allergy test $(\mathrm{P}=0.04)$. Thirty six subjects $(72 \%)$ had concommitantly hypersensitivity reactions to other allergens, among which $24(67 \%)$ to other drugs and $13(35 \%)$ to inhalated allergens. Positive family history of penicillin allergy was found in $13(26 \%)$ subjects.

Conclusion: Patients with hypersensitivity reactions to penicillins should be tested to exclude or prove allergy. Our results have shown that specific $\mathrm{IgE}$ antibodies to penicillins as an in vitro methods have very low sensitivity. Approximately one third of our subjects had positive in vivo test to penicillins. Angioedema as clinical manifestation was found significantly more in patients with positive test outcome. On the other hand, the outcome was not correlated to the time interval between the initial reaction and testing. This is a small pilot study and further research is needed.

36

Selection of antibiotic therapy regimens for infections caused by problematic pathogens

Albina Zvegintseva ${ }^{1,2}$, Lyudmila Kulagina ${ }^{1,2}$, Inna Valiullina ${ }^{1}$,

Maxim Maksimov ${ }^{\mathrm{T}}$

${ }^{1}$ Republican Clinical Hospital Ministry of health Republic of Tatarstan, Kazan, Russian Federation

${ }^{2}$ Kazan State Medical Academy - Branch Campus of the Federal State Budgetary Educational Institution of Further Professional Education «Russian Medical Academy of Continuous Professional Education» of the Ministry of Healthcare of the Russian Federation, Kazan, Russian Federation

Introduction: Antibiotic resistance is the phenomenon of resistance of an infectious pathogen strain to the action of one or more antibacterial drugs, immunity and resistance of microbial culture to the action of an antibacterial substance. The most problematic among the pathogens of nosocomial infections are microorganisms that have super capacity to form multiple resistance to major classes of antimicrobial drugs. The ESKAPE acronym reflects the ability of these microorganisms to "avoid" killing by antibiotics by developing resistance mechanisms, which leads to high morbidity, longer hospitalizations, worsens prognosis, increases mortality and leads to higher health care costs.

Objective: The aim of this work was to determine the trend of prevailing "problem" microflora and develop a strategy for empirical antibiotic therapy in severe nosocomial infectious and inflammatory processes.

Materials and Methods: We analyzed the main groups of pathogens of hospital infections in the dynamics for 2012-2020. The ratio of positive cultures to the total number of tested samples was taken for analysis. The isolation, identification and determination of antibiotic sensitivity were performed using conventional methods using mass spectrometry.

Results: There is a stable excretion rate of Acinetobacter baumannii (2012-2.18\%; 2013-3.23\%; 2014-4.20\%; 2015-6.44\%; 2016-5.63\%; 2017-6.35\%;2018-6.05\%;2019-6.39\%;2020-5.96\%). and Klebsiella pneumoniae (2012-3.85\%; 2013-5.94\%; 2014-8.76\%; 2015-10.98\%; 2016-11.28\%; 2017-12.0\%;2018-13.71\%;2019-12.09\%;2020-12.68\%) with high levels of resistance. Increased significance of Acinetobacter baumannii in intensive care units and neonatal intensive care units is most likely associated with artificial lung ventilation. Klebsiella pneumoniae has been a leader in intensive care, burn and purulent surgery departments of the Republican Clinical Hospital of the Ministry of Health of the Republic Tatarstan for many years. In daily practice, physicians face great difficulties in selecting antibiotic therapy for infections caused by panresistant strain of Klebsiella pneumoniae.

Conclusion: Microbiological monitoring of problem microorganisms allows to consider the most effective schemes of antimicrobial therapy. High resistance of hospital strains of these pathogens of purulent inflammatory diseases has to be overcome by a combination of 2-3 antimicrobials with natural activity against them (carbapenems, inhibitor-proof cephalosporins, aminoglycosides). Doses are increased to the maximum allowable and a prolonged infusion regimen is used to put the most pressure on resistant flora. The use of new inhibitor-proof cephalosporins leads to positive dynamics of clinical and laboratory data of patients and gives hope for good results in the future if they are used rationally.

With the support of the administration of the Republican Clinical Hospital of the Ministry of Health of the Republic Tatarstan for solving the problem of antibiotic resistance in the hospital - a strategy for its containment has been developed, which includes several directions:

-Continuous microbiological monitoring, prompt provision of bacteriological results for rational choice of adequate antibiotic therapy;

-Epidemiological surveillance of hospital infections, especially in intensive care units;

-Strict control of the use of antibiotics by clinical pharmacologists in the hospital allows to ensure the reduction of the frequency of their irrational use and restrains the spread of resistant strains of microorganisms.

40

Assessment of the practice of using antibiotics in the pulmonary department of a children's hospital

M.Sc., MD, Ainur Ikhambayeva ${ }^{1}$, D.Sc., MD Nurgul ALDIYAROVA ${ }^{2}$ $\mathrm{PhD}, \mathrm{MD}$ Larisa Makalkina ${ }^{1}$

${ }^{1}$ Medical University Astana, Nur-Sultan, Kazakhstan

${ }^{2}$ Professional Association of Clinical Pharmacologists and Pharmacists, Nur-Sultan, Kazakhstan

Introduction: One of the main reasons for the rise of antibiotic resistance in healthcare settings is irrational use of antibiotics.

Objectives: Obtain objective data on antibiotic use.

Materials and methods: The medical records of patients / children of the pulmonary department receiving antibiotic therapy were analyzed. Appropriateness of antimicrobial therapy was assessed using the following indicators: accuracy of choice of an antibiotic for empiric therapy, adequacy of the dose regimen, the rationality of combination, and accordance of the choice of antibiotic with national clinical protocols.

Results: The analysis of antibiotic prescriptions showed that the doctors of the children's hospital used 15 types of antibiotics from 6 different 
pharmacological groups (penicillins, cephalosporins, carbapenems, macrolides, aminoglycosides, sulfonamides). The analysis included 924 antibiotic prescriptions over 4 months (May to August, 2019). Antibiotic prescriptions as initial therapy were divided according to class in the following proportions: cephalosporins (cefuroxime, ceftriaxone, cefotaxime, ceftazidime, cefepime) - 79.2\%; aminoglycosides (gentamicin, amikacin) - 8.4\%; penicillins (ampicillin, amoxicillin) -7,2\%; macrolides (azithromycin, clarithromycin) - 4.3\%; carbapenems (imipenem, meropenem) $-0.2 \%$; sulfonamides (co-trimoxazole) - $0.1 \%$. For lower respiratory tract infections, third-generation cephalosporins, in particular ceftriaxone and ceftazidime, were prescribed most frequently. Analysis of prescriptions showed that parenteral administration of antibiotics was preferred in $93.2 \%$ of cases; a combination of two antibiotics was chosen for initial therapy in $5.4 \%$ of cases. Mostly, in community-acquired pneumonia, the following drugs were irrationally chosen as an initial therapy: ceftazidime, cefotaxime, cefepime, amikacin, gentamicin, clarithromycin, co-trimoxazole. The inadequacy of the dose regimen was also observed; errors in choosing a single dose were encountered when prescribing cefuroxime, ceftazidime and gentamicin. In $11.3 \%$ of cases, unreasonably low or high doses were prescribed. Frequency of administration was not accurate in $11.5 \%$ of cases. It was mainly encountered among cefuroxime, ceftriaxone, ceftazidime, cefotaxime and gentamicin prescriptions.

Conclusion: The analysis of the rationality of antibiotics use showed a number of defects: irrational choice of antibiotics for empiric therapy, unreasonable use of antibiotic combinations, non-compliance with the dose regimen by hospital doctors.

\section{1}

Case Study of Vancomycin Penetration into Brain Extracellular Fluid in Patients with Meningitis After Neurosurgery Mrs. Skaiste Kasciuskeviciute ${ }^{1}$, Mr. Karolis Bareikis ${ }^{1}$, Dr. Tomas Tamosuitis ${ }^{1}$, Prof. Romaldas Maciulaitis ${ }^{1}$ ${ }^{1}$ Lithuanian University Of Health Sciences, Kaunas, Lithuania

Introduction: The efficacy of antimicrobials (AMs) depends on their capacity to achieve adequate concentration at the site of infection. The central nervous system (CNS) distribution of AMs is limited by the presence of the blood-brain barrier; therefore, plasma drug levels may not reflect brain extracellular fluid (ECF) levels which are considered the target site in case of meningitis. There is limited information about the pharmacokinetics (PK) after neurosurgical operation. We investigated CNS distribution of vancomycin (VAN) that is commonly used to treat neurosurgery-related bacterial meningitis caused by gram-positive pathogens.

Objectives: To describe VAN penetration into brain ECF by microdialysis (MD) in patients with bacterial meningitis after neurosurgery.

Methods: This was a case study of two patients diagnosed with postoperative bacterial meningitis and treated with VAN were included in the study. Both patients underwent routine monitoring using brain MD for determination of metabolism parameter concentrations. ECF, blood and CSF (where available) samples were collected simultaneously at steady state. The time points for sample collection were guided by the rate of MD microvials filling. To account for the inter-day variability samples were collected over one dosing interval on two consecutive days. VAN levels were determined by the turbidimetric inhibition immunoassay method. Results are provided in descriptive statistics.

Results: The first patient was 28 years old male with congenital cerebral palsy, transverse myelitis at the conus medullaris level and epilepsy who underwent surgery for frontobasal encephalocele. Postoperative course was complicated by obstructive hydrocephalus $(\mathrm{OH})$ and staphyloccocal meningitis. Since $\mathrm{OH}$ was managed by an external ventricular drain, VAN levels in CSF were also available for this patient. The second patient was 69 years old male with chronic subdural haematoma requiring surgical removal. Three weeks after surgery the patient was diagnosed with culture negative bacterial meningitis and prescribed VAN as a part of empirical treatment. His renal function was declining constantly, therefore only one full dosing interval was used to describe PK of VAN. The patient died as a result of progressive infection.

Concentration-time profile of VAN in the brain ECF was flat for both patients. ECF levels did not follow plasma profile and increased during period between dosages. ECF levels did not achieve higher than $43.7 \%$ of plasma levels, which was observed at the end of dosing interval. VAN levels in the ECF were also lower than those measured in the CSF and accounted for $24.5 \%$ and $42.6 \%$ of CSF levels at the beginning and the end of dosing interval, respectively. In terms of the outcomes, the patient was successfully cured from the infection. In the second case, the ECF-toplasma ratio was much lower and ranged from $0.0 \%$ to $3.4 \%$. The patient died as a result of progressive infection 8 days after the initiation of antibacterial treatment.

Conclusions: The levels of vancomycin were lower in the brain ECF than CSF or plasma and varied significantly in two patients. Further investigation is needed to better characterized vancomycin penetration into brain $\mathrm{ECF}$ and identify factors impacting different penetration ratios.

\section{2}

Vancomycin therapeutic monitoring in neutropenic patients MD Olga Hladun ${ }^{1,2}, \mathrm{PhD}, \mathrm{MD}$ Clara Pérez-Mañá ${ }^{1,2}, \mathrm{PhD}, \mathrm{MD}$ Esther Papaseit $^{1,2}$, MD Melani Nuñez ${ }^{1,2}, \mathrm{PhD}$, MD Magí Farré ${ }^{1,2}$

${ }^{1}$ Department of Clinical Pharmacology, Hospital Universitari Germans Trias i Pujol and Institut de Recerca Germans Trias i Pujol (IGTP), Badalona, Spain

${ }^{2}$ Department of Pharmacology, Therapeutics and Toxicology, Universitat Autònoma de Barcelona (UAB), Cerdanyola del Vallès, Spain

Introduction: Febrile neutropenia (FN) is a life-threatening condition in hematological patients that requires immediate attention due to the high mortality rates. A glycopeptide has to be associated to cover suspected methicillin-resistant Staphylococcus aureus infection (MRSA), in those patients with persistent fever initially treated with $\beta$-lactam with antipseudomonal activity. It has been reported previously that patients with neutropenia have higher vancomycin clearance and need higher doses $(25 \%)$ to achieve therapeutic concentrations. Protocolized regimens of vancomycin between 15-20 $\mathrm{mg} / \mathrm{kg}$ given every $8-12$ hours, may not achieve recommended therapeutic trough concentrations $(15-20 \mathrm{mg} / \mathrm{L})$.

Objectives: We aimed to assess the concentrations of vancomycin exposure in hematological patients with $\mathrm{FN}$, in order to know the prevalence of subtherapeutic levels and the increases in dose needed to obtain recommended plasma concentrations.

Methods: We conducted a retrospective study of all hematological patients with FN treated with vancomycin monitored by our clinical pharmacology service from January to December 2020. Demographic variables, renal function (CKD-EPI), vancomycin initial dose prescribed by their physician, dose adjustments and trough levels have been collected. A trough of $15-20 \mathrm{mg} / \mathrm{L}$ has been considered a therapeutic concentration ( $\geq 10 \mathrm{mg} / \mathrm{L}$ is a broader approach).

Results: Our sample had 41 patients, $68 \%$ of men, with an average age and weight of 50 years and $72.6 \mathrm{~kg}$, respectively. Average vancomycin initial daily dose was $2,244 \pm 435 \mathrm{mg}(14.7 \mathrm{mg} / \mathrm{kg} / 12 \mathrm{~h}$ in 33 patients and $13.1 \mathrm{mg} / \mathrm{kg} / 8 \mathrm{~h}$ in 8 patients). Average renal glomerular filtration was $112 \mathrm{~mL} / \mathrm{min} / 1.73 \mathrm{~m} 2$ and first trough was 7.2 $\pm 3.6 \mathrm{mg} / \mathrm{L}(\mathrm{n}=39)$ and only one patient reached $>15 \mathrm{mg} / \mathrm{L}(26 \%$ achieved 10-14 mg/L). After dose adjustment to an average dose of $3,041 \pm 602 \mathrm{mg}(19.3 \mathrm{mg} / \mathrm{kg} / 12 \mathrm{~h}$ in 15 patients and $15.3 \mathrm{mg} / \mathrm{kg} / 8 \mathrm{~h}$ in 22 patients) the second trough was $13.1 \pm 4.7 \mathrm{mg} / \mathrm{L}(\mathrm{n}=30)$. Although dose was increased a mean of $39 \%$ in 34 patients, $62 \%$ still had suboptimal concentrations $(23 \%<10 \mathrm{mg} / \mathrm{l})$. Most of the remaining patients on treatment required further adjustments to achieve therapeutic concentrations. 
Conclusions: According to the data obtained, our NF patients are prescribed lower doses than those recommended by weight in normal renal function. In addition, as described above, a higher clearance of vancomycin may be another factor to consider in the failure to obtain the desired vancomycin levels. Furthermore nearly all of them did not achieve optimal concentrations in the first trough (after 2 days of treatment). The use of a loading dose or prescription of initial doses of vancomycin of $20 \mathrm{mg} / \mathrm{kg} / 12 \mathrm{~h}$ or $15 \mathrm{mg} / \mathrm{kg} / 8 \mathrm{~h}$ could be a useful strategy to achieve early target, accompanied by therapeutic monitoring to maintain vancomycin levels on target and reduce the risk of nephrotoxicity.

\section{6}

Is antibiotic use proper in rural areas of Transylvania?

Dr. Erika Ban ${ }^{1}$, dr. Zoltan Köllö ${ }^{1}$, Student Andrea-Maria Kalapacs ${ }^{1}$, Prof.Dr. Attila Brassai ${ }^{1}$, Dr. Erika Ban ${ }^{1}$

${ }^{1}$ Umfst, Tirgu Mures, Romania

Introduction: The inappropriate use of antibiotics is the main cause of antimicrobial resistance, which showes a worsening tendency these years. The World Health Organization noted the complexity of this emerging situation and has implemented a coordinated action plan all over the world.

Objectives: We have performed a pharmacoepidemiologic evaluation about antibiotic drug use in the adult popultaion of rural Transylvania in aim to detect the weak links in the rational antibiotic use, which characterize the studied region. We have to assess the general knowledge about antimicrobial agents, the factors that compromise the compliance, and the inaccurate information of the population.

Methods: We have performed an observational cross-sectional, epidemiological study based on questionnaires addressed to adult subjects living in rural Transylvania. During the survey we followed the Declaration of Helsinki. Statistical analysis was performed using Excel and SPSS Statistics.

Results: We performed a complete analysis of demographic data, other demographical variables of our research sample, including marital status, education, work status and number of maintained persons

In order to investigate the purpose of our study, more exactly, the knowledge about antibiotic usage, we studiedhow many of the respondents gave the correct answers to the particular items aimed this aspect. Results returned that: $65 \%$ had an adequate knowledge of the antibiotic indications, $84 \%$ recognized the medical prescription as important indication to start the antibiotics, $45 \%$ admitted in the last question to have taken antibiotic for self-medication, $51 \%$ ended prematurely the antibiotic treatment when their symptoms disappeared.

Conclusions: Our study revealed that we have results comparable to the systematic report published in 2016 containing data from Europe, Asia and North America which stated that $70 \%$ of the adults heard of antibiotic resistance, $60 \%$ of the respondents attributed this phenomenon to the excessive antibiotic use, and $62 \%$ attributed it to the fact that the prescribed antibiotic treatments were not completed. This report confirmed the need of intervention and the formulation of action plans regulating the access to antibiotics with stronger regulatory frameworks, improving national surveillance on consumption and supplementing the correct information amount about the rational antibiotic use.

\section{9}

\section{VORICONAZOLE vS ISAVUCONAZOLE INFLUENCE ON TRANSPLANT RECIPIENTS UNDERTAKING TACROLIMUS Md Rita Nogueiras Álvarez ${ }^{1}$, Md David Valencia López ${ }^{1}$,} Md Diana Zulema Cuéllar Gómez ${ }^{1}$, Víctor Manuel Mora Cuesta ${ }^{2}$, Miriam Sánchez Escamilla ${ }^{3}$, Md Iván Mazón Maraña ${ }^{1}$,

Md Beatriz Alonso Gómez ${ }^{1}$, Md Andrea Bautista Blázquez ${ }^{1}$,

Md María Mercedes Fuentes Rodríguez ${ }^{1}$,

Md Gorka Gaztelumendi Martín ${ }^{1}$, María Blanca Sánchez Santiago ${ }^{1}$,
Noelia Vega Gil ${ }^{1}$, María de los Ángeles de Cos Cossío ${ }^{1}$, José Manuel Nicolás de la Puente ${ }^{1}$, María del Mar García Sáiz

${ }^{1}$ Clinical Pharmacology. Hospital Universitario Marqués de Valdecilla, Santander, Spain

${ }^{2}$ Pneumology. Hospital Universitario Marqués de Valdecilla, Santander, Spain

${ }^{3}$ Hematology. Hospital Universitario Marqués de Valdecilla, Santander, Spain

INTRODUCTION: Invasive fungal infections are an important cause of morbimortality in immunocompromised patients such as transplant recipients (TR). When prescribing antifungal drugs (AFD) to patients undergoing treatment with tacrolimus (TAC), PK interactions should be taken into account. Triazole AFD are inhibitors of CYP3A, a cornerstone in TAC metabolism.

OBJECTIVES: To analyze differences in the influence of triazole AFD (voriconazole-VOR and isavuconazole-ISA) on TAC blood levels during the coadministration and afterwards the azole withdrawn.

METHODS: We retrospectively analyzed 37 patients (solid organ transplants $=25$; haematopoietic stem cell transplants $=12$ ) who received immunosuppressant therapy with TAC and an AFD (VOR or ISA), from October2017 to February2021. We collected demographic characteristics, cause for AFDs prescription (prophylaxis: $16.2 \%$ /treatment: $83.8 \%$ ), and type of triazole. We analyzed the TAC through blood concentration normalized by its daily dose (C/D ratio) at different time-points (before AFD prescription, at the moment of achieving maximum $\mathrm{C} / \mathrm{D}$ ratio after antifungal prescription, and after AFD withdrawn). We also recorded adverse drug reactions (ADR) identified during the treatment.

RESULTS: Twenty-five patients received VOR and 12 patients ISA ( 24 with another AFD simultaneously). The mean C/D ratio of TAC increased from 2.93 to 11.80 during coadministration of VOR, and from 1.88 to 3.45 during ISA. TR who received VOR showed a greater increase in their maximum TAC C/D ratio when compared with ISA, by a mean of 6.2 vs 2.2 -fold $(\mathrm{p}=0.002)$, and besides, they took less time to reach that maximum (11 days with VOR vs 17.5 days with ISA)

ADR were more frequent in the VOR group (60\% vs 33\%). The most frequent ADR were hepatotoxicity, followed by renal, gastric and neurologic impairment. Due to toxicity, antifungal treatment was discontinued in up to 9 patients ( 8 from VOR, 1 from ISA).

CONCLUSION: When prescribing triazole AFD in TR already on TAC, an increase on blood levels should be expected due to the inhibition of intestinal and hepatic CYP3A isoenzymes.

ISA seems a good alternative to VOR, as its influence on TAC blood levels is lower and shows fewer toxicities.

However, therapeutic drug monitoring of TAC should be performed both during antifungal treatment and when it is discontinued to optimize drug dosage for achieving the recommended immunosuppressant levels and try to avoid toxicities.

85

\section{NEBULIZED VORICONAZOLE IN LUNG} TRANSPLANTATION: TWO CASE REPORTS

Md Rita Nogueiras Álvarez ${ }^{1}$, Md David Valencia López ${ }^{1}$,

Víctor Manuel Mora Cuesta ${ }^{2}$, Md Diana Zulema Cuéllar Gómez ${ }^{1}$, Noelia Vega Gil ${ }^{1}$, María Blanca Sánchez Santiago ${ }^{1}$, María de los Ángeles de Cos Cossío ${ }^{1}$, Md Iván Mazón Maraña ${ }^{1}$, Md Beatriz Alonso Gómez ${ }^{1}$, Md Andrea Bautista Blázquez ${ }^{1}$, Md María Mercedes Fuentes Rodríguez ${ }^{1}$, Md Gorka Gaztelumendi Martín, David Iturbe Fernández², Sonia María Fernández Rozas ${ }^{2}$, José Manuel Cifrián Martínez², José Manuel Nicolás de la Puente ${ }^{1}$, María del Mar García Sáiz ${ }^{1}$

${ }^{1}$ Clinical Pharmacology. Hospital Universitario Marqués de Valdecilla, Santander, Spain 
${ }^{2}$ Pneumology. Hospital Universitario Marqués de Valdecilla, Santander, Spain

INTRODUCTION: Invasive fungal infections (IFI) are an important cause of morbimortality in immunocompromised patients. Lung transplant recipients (LTR) are a vulnerable group due to the intense immunosuppression required.

OBJECTIVES: While analysing the impact of antifungal drugs on tacrolimus blood levels in transplant recipients of our center, we identified two LTR that were being treated with inhaled voriconazole. We made a bibliographic review about the topic, as this formulation is less used in clinical practice.

METHODS: We report two clinical cases of LTR with IFI undergoing nebulized voriconazole treatment.

RESULTS: We found two cases of LTR with fungal infections undertaking nebulized voriconazole.

Patient A) Woman with single left lung transplant for interstitial lung disease (ILD) with isolation of Aspergillus in respiratory bronchoalveolar lavage (BAL) and several sputum specimens. She received conventional antifungal nebulized treatment with nebulized liposomal amphotericin B; but despite this, the chest $\mathrm{CT}$ scan revealed a mycetoma image that continuously increased its size on following controls, so nebulized treatment with voriconazole was prescribed. After three months of treatment, CT scan image showed no progression. Currently under nebulized voriconazole treatment, with no significant influence on its tacrolimus blood levels, and good tolerance to the antifungal.

Patient B) Woman with double lung transplantation for cystic fibrosis with chronic fungal infection with Scedosporium prolificans in BAL and various sputum specimens. Before transplantation she had already undergone antifungal treatment with nebulized liposomal amphotericin B and oral posaconazole, terbinafine and voriconazole. Due to resistance to treatment and progressive intolerance to high doses of oral voriconazole, it was decided to start nebulized treatment. On the first administrations she reported somnolence and nausea, but then they disappeared. Now ongoing treatment with good tolerance and stable tacrolimus blood levels.

CONCLUSION: Nebulized voriconazole has been studied in murine models, but there are few reports of its clinical use. Nebulized voriconazole: $40 \mathrm{mg} / 6.4 \mathrm{ml}$ each day $(6.25 \mathrm{mg} / \mathrm{mL})$ can be an option for treating severe and chronic pulmonary fungal infections in immunocompromised patients. Due to its targeted pulmonary delivery by aerosolization, this formulation allows for higher concentrations at the primary site of infection.

\begin{tabular}{|c|c|c|}
\hline & Patient A & Patient B \\
\hline Type of lung transplant & $\begin{array}{l}\text { Left single lung transplant for } \\
\text { interstitial lung disease (ILD) }\end{array}$ & $\begin{array}{l}\text { Double lung transplantation for cystic } \\
\text { fibrosis }\end{array}$ \\
\hline Diagnosis & Invasive fungal infection (IFI) & Chronic pulmonary fungal infection \\
\hline Isolated microorganism & $\begin{array}{l}\text { Aspergillus fumigatus } \\
\text { Aspergillus flavus }\end{array}$ & Scedosporium prolificans \\
\hline $\begin{array}{l}\text { Cause for nebulized } \\
\text { voriconazole prescription }\end{array}$ & $\begin{array}{l}\text { chest CT scan mycetoma image } \\
\text { progression }\end{array}$ & resistance to previous treatments \\
\hline $\begin{array}{l}\text { Other antifungal drugs } \\
\text { used (different from nebulized } \\
\text { voriconazole) }\end{array}$ & nebulized liposomal amphotericin B & $\begin{array}{l}\text { nebulized liposomal amphotericin B } \\
\text { PREVIOUSLY: oral posaconazole, oral } \\
\text { terbinafine, oral voriconazole }\end{array}$ \\
\hline $\begin{array}{l}\text { Days until maximum } \\
\text { tacrolimus C/D ratio was } \\
\text { achieved (after nebulized } \\
\text { voriconazole was prescribed) }\end{array}$ & 224 days & 56 days \\
\hline $\begin{array}{l}\text { Increment ratio (between } \\
\text { maximum tacrolimus } C / D \text { ratio } \\
\text { after nebulized voriconazole } \\
\text { prescription and the tacrolimus } \\
C / D \text { ratio before its } \\
\text { prescription) }\end{array}$ & 1,70 & 2,42 \\
\hline $\begin{array}{l}\text { Adverse drug reactions } \\
\text { identified afterwards } \\
\text { nebulized voriconazole } \\
\text { prescription }\end{array}$ & post-administration headache & somnolence, nausea \\
\hline Evolution & $\begin{array}{l}\text { Ongoing nebulized voriconazole } \\
\text { treatment with good tolerance and } \\
\text { appropriate tacrolimus blood levels }\end{array}$ & $\begin{array}{l}\text { Ongoing nebulized voriconazole } \\
\text { treatment with good tolerance and } \\
\text { appropriate tacrolimus blood levels }\end{array}$ \\
\hline
\end{tabular}

\section{Poster Session 3a - Chronic conditions/COVID-19}

10

Multidrug therapy in patients with hypertension with multiple associated diseases

Andrea-Mária Kalapács ${ }^{1}$, Coauthor,

Coordonating author Erika-Gyöngyi Bán ${ }^{1}$,

Coauthor Eszter Anna Dho-Nagy ${ }^{1}$, Coauthor Attila Brassai ${ }^{1}$,

Coauthor Csongor Szabados ${ }^{1}$

${ }^{1}$ University of Medicine, Pharmacy, Science and Technology of Tg.

Mures, Targu Mures, Romania

Introduction: Pharmacological therapy used in the treatment of adult patients with hypertension and other comorbidities has an influence on the cardiovascular markers.

Objectives: The objective of the present study consists of the evaluation of the medical condition in hypertensive patients and the evaluation of treatment based especially on the number and groups of drugs used simultaneously.

Methods: We have performed a non-interventional, cross-sectional retrospective study regarding the pharmacotherapy of 200 adult patients with primary hypertension.

Results: In order to evaluate the medical condition of the research population we proceeded a descriptive analysis of all the diseases from the medical files. The most frequent diseases are cardiac ischemia $(61.5 \%)$, valvular diseases $(58.5 \%)$ and other cardiovascular illnesses $(62.5 \%)$.

Data analysis indicates significant correlation between gender and pacemaker wear, bypass wear, arteriopathy and varicose veins. In this research male patients are the affected majority, excepting the varicose veins, which is found mostly in women. These results mean that gender could be an influence factor in this kind of cardiovascular disease. Age can be also an influencing factor considering that significant correlations were obtained between age and arrhythmia and valvular diseases.

In terms of drug interaction, we obtained significant correlations between the number of drugs and cardiac ischemia, valvular diseases and heart failure, meaning that number of drugs could influence this category of diseases. The factorial analysis indicates that IECA and AINS interaction influences significant the patient's blood potassium level $(\mathrm{F}=4.6$; $\mathrm{Sig}=0.03)$. None of the drugs indicates determines significant changes of potassium.

Conclusion: We observed similar results in our study as they are present in worldwide literature. Taking into consideration all the limitations of this study and the specificity of the cases we can although conclude that detailed analysis of the treatment can give us further guidelines how the therapy can be improved to provide a better control, compliance and a lower incidence of unwanted drug-drug interactions.

\section{9}

IMPACT OF COVID-19 ON CYTOCHROMES P450 ACTIVITY ASSESSED BY THE GENEVA COCKTAIL

Camille Lenoir $^{1}$, Dr Jean Terrier ${ }^{2}$, Dr Yvonne Gloor ${ }^{1}$, Dr François Curtin $^{1}$, Dr Victoria Rollason ${ }^{1}$,

Prof Jules Alexandre Desmeules ${ }^{1}$, Prof Youssef Daali ${ }^{1}$,

Prof Jean-Luc Reny ${ }^{2}$, Prof Caroline Flora Samer ${ }^{1}$

${ }^{1}$ Division of Clinical Pharmacology and Toxicology, Department of Anaesthesiology, Pharmacology, Intensive Care and Emergency Medicine, Geneva University Hospitals, Geneva, Switzerland

${ }^{2}$ Division of General Internal Medicine, Department of Medicine, Geneva University Hospitals, Geneva, Switzerland

Introduction: Coronavirus Disease 2019 (COVID-19), caused by SARS-CoV-2 infection, is characterized by a severe acute respiratory 
syndrome and a strong underlying inflammatory state in severe cases, where IL-6 plays a key role. We have previously demonstrated that acute inflammation (hip surgery) modulates cytochromes P450 (CYP) activities in an isoform-specific manner. Therefore, we hypothesized that SARSCoV-2 infection could also have an impact on CYPs activities and thus on the pharmacokinetics (PK) of drugs received by COVID-19 patients.

Objectives: Our study aimed to evaluate the impact of COVID-19 on the activity of the six main human CYP.

Methods: This prospective observational study was conducted in $30 \mathrm{pa}-$ tients hospitalized at Geneva University Hospitals (Switzerland) with a diagnosis of moderate to severe COVID-19. They received the Geneva phenotyping cocktail (containing the following probe substrates: caffeine, bupropion, flurbiprofen, omeprazole, dextromethorphan and midazolam) orally twice: during the first $72 \mathrm{~h}$ of hospitalization and after three months. Capillary blood samples were collected $2 \mathrm{~h}$ after the cocktail was taken to assess the metabolic activities of CYP1A2, 2B6, 2C9, 2C19, 2D6 and 3A. CRP, IL- 6 and TNF- $\alpha$ levels were also measured in blood.

Results: Mean CRP levels were $104.3 \mathrm{mg} / \mathrm{L}$ and $2.8 \mathrm{mg} / \mathrm{L}$ during and three months after COVID-19 respectively. CYP3A, CYP2C19 and CYP1A2 metabolic ratios (MR) decreased by $62.5 \%$ ( $\mathrm{p}=0.01$ ), $23.0 \%$ $(\mathrm{p}=0.02), 50.0 \%(\mathrm{p}=0.005)$ respectively during SARS-CoV-2 infection while CYP2C9 MR increased by $142.0 \%$ ( $\mathrm{p}=0.002$ ). SARS-CoV-2 infection had no impact on CYP2B6 $(\mathrm{p}=0.42)$ and CYP2D6 $(\mathrm{p}=0.14)$ MRs.

Conclusion: CYP activities were modulated in an isoform specific manner by SARS-CoV-2 infection and subsequent inflammation. The plasma concentration of CYP substrates could therefore be clinically significantly influenced by SARS-CoV-2 infection and modulate their benefit/risk ratio in this setting.

\section{8}

Managing shortage of intensive care drugs during the COVID-19 pandemic in Catalonia PhD Marta Roig ${ }^{1}$, MSc Laura Guarga ${ }^{1}$, MSc Salvador Cassany ${ }^{2}$, MD, PhD César Hernández ${ }^{3}, \underline{\text { MD, PhD Caridad Pontes }}{ }^{1}$

${ }^{1}$ Catalan Health Service, Barcelona, Spain,

${ }^{2}$ Catalan Health Department, Barcelona, Spain

${ }^{3}$ Spanish Agency of Medicines and Medical Devices, Madrid, Spain

Introduction: The first wave of the COVID-19 pandemic in Spain accounted 215,216 confirmed cases and 24,824 deaths between Jan 31st to May 2nd 2020. Since approximately $5 \%$ of patients infected with SARS CoV-2 received mechanical ventilation, there was a significant demand for sedation and analgesia drugs. During 3 weeks, Spain had risk of shortage of ICU medicines and a number of measures were taken.

Objectives: To ensure supplies of a list of essential medicinal products required to treat patients receiving intensive care for COVID-19. To define therapeutic contingencies in case of stock breakage.

Methods: Emergency laws were issued at National level determining regulatory requirements for frequent information on manufacturing and controlled distribution of medicines with highest demand (cisatracurium, dexmedetomidine, midazolam, propofol, rocuronium, and IV azithromycin). The Spanish Agency of Medicines and Medical Devices determined an equitable controlled distribution of the available units of medicines to each Spanish Region according to daily reporting of occupied critical beds. At regional level, the Catalan Health Department centralised hospital demands and assigned the maximum number of available units that could be purchased per each of 57 hospitals in the region holding critical beds. Also, a tool to calculate standard medication needs was created, based on the number of occupied critical beds, and included calculation of therapeutic contingencies in case of stock breakage.

Results: At the worst of the outbreak, up to 2,081 simultaneous beds in Catalonia were providing respiratory support to patients with critical acute respiratory failure. Daily controlled distribution managed about 750,000 units of essential medicines. None of the 57 sites had lack of supply with clinical repercussions. A centralised stock and distribution centre for
Catalan hospitals was contracted and enabled to operate. A tool to forecast hospital needs according to the number of beds occupied by patients with critical COVID-19, average daily dose requirerments in sequential clinical phases and contingency drug regimes was created, to support medicines allocation.

Conclusion: The implemented measures in Spain and Catalonia were able to avoid lack of supply, prevented stockpiling and ensured that hospitals had the medicines required to treat their patients.

35

An Observational Study in India on Steroid Sparing Agents in Frequently Relapsing, Steroid Dependent and Steroid Resistant Nephrotic Syndrome children

Dr Sabnam Begam ${ }^{1}$

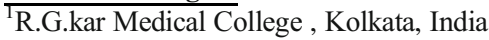

Introduction: Nephrotic syndrome is a common pediatric kidney disease characterized by leakage of protein from the blood into the urine through damaged glomeruli. It. It is a common clinical condition in Asian children. It is estimated that about $80 \%$ of children with idiopathic nephrotic syndrome will respond to corticosteroid treatment with complete resolution of proteinuria and edema. In some case where they are used in high doses and prolonged period of time side effects of steroids can occur, including cataract, growth failure, obesity, hypertension, osteoporosis, hirsutism etc. To avoid steroid toxicity, several steroid sparing agents have been proposed to treat these children. Several studies indicate that steroid sparing agents (SPAs) like levamisole, cyclophosphamide, mycophenolate mofetil (MMF), cyclosporine, tacrolimus, or rituximab etc. can be used for the treatment of Frequently Relapsing Nephrotic Syndrome (FRNS), Steroid Dependent Nephrotic Syndrome (SDNS) and Steroid Resistant Nephrotic Syndrome (SRNS). There are different guidelines available worldwide but no particular standard guidelines to be followed universally. In this study an attempt was made to find out the lacunae in the recommendation practices, and the impact of such gaps on the outcomes of the patients. It was apprehended that the findings generated would enlighten us on this controversial area and will enable us to delineate the treatment management.

Objective: The present study tried to assess the relative effectiveness, safety and impact on quality of life after usage of most commonly steroid sparing agent SSA in children with FRNS, SDNS and SRNS

Methodology: A multi-centered, prospective observational study was conducted in pediatric nephrology OPD of two tertiary care hospitals in Kolkata over a period of 24 months. All consecutive FRNS, SDNS and SRNS children started on SSA were enrolled and followed up for at least 6 months. Records of clinical examination, laboratory tests were collected and measured at the baseline and regular intervals. Subjects were assessed for quality of life using Family Impact Module and standard PedsQL questionnaire and statistically analyzed.

Results: A total 104 patients were screened, examined and enrolled. As levamisole was the most commonly used SPA (93.27\%) in comparison to Cyclophosphamide (4.81\%) and MMF (1.92\%), we had to restrict our analysis on levamisole therapy. Nausea $(57.73 \%)$ was the most common side effect with levamisole therapy though found self limiting in nature. Serious adverse event were documented in $18.56 \%$ subjects, however the cause can also be attributed to the disease progression and noncompliance, thus definite causality for the same cannot be established. There were significant changes in both ESRD and FIM total scale score at 6 month FU from baseline of 97 levamisole receiving subjects $(\mathrm{P}<0.01)$ and 12 months FU of 43 same subjects from baseline value $(\mathrm{P}<0.05)$.

Conclusion: Levamisole being a very efficacious, safe, improving quality of life in such children and their parents as well as easily affordable (hospital supply) drug, should be used as an initial first line SSA in treating FRNS and SDNS children 
69

THE MOST FREQUENT COMPLICATIONS IN PATIENTS WITH NEW COVID-19 CORONAVIRUS INFECTION

Albina Zvegintseva ${ }^{\mathbf{1}, 2}$, Irina Burashnikova ${ }^{2}$, Maxim Maksimov ${ }^{1,2}$

${ }^{1}$ Republican Clinical Hospital Ministry of health Republic of Tatarstan, Kazan, Russian Federation

${ }^{2}$ Kazan State Medical Academy - Branch Campus of the Federal State Budgetary Educational Institution of Further Professional Education «Russian Medical Academy of Continuous Professional Education» of the Ministry of Healthcare of the Russian Federation, Kazan, Russian Federation

Introduction: Today the prevention of the disease and search for effective treatment of COVID-19 and its complications is a serious problem for population, scientific communities, health care systems and other departments all over the world. Besides high contagiousness of the virus, possibility of severe course of the disease in some patients, various complications and long rehabilitation process caused by new coronavirus infection COVID-19 become a serious problem.

Objective: to analyze the most frequent complications after the new coronavirus infection COVID-19.

Materials and Methods: Questionnaire survey of patients who had undergone new coronavirus infection COVID-19 with a positive PCR test for detection of SARS-Cov2 RNA.

Results of the study: 200 patients aged 18-68 years (mean age 39.6 years), including $158(79 \%)$ women and $42(21 \%)$ men participated in the study. According to the results of the questionnaire, 116 (58\%) patients had mild COVID-19 infection, 74 (37\%) had moderate infection, $8(4 \%)$ had severe infection, and $2(1 \%)$ patients had extremely severe infection. $162(81.4 \%)$ were on outpatient treatment, $38(18.6 \%)$ were on inpatient treatment, of which $17(8.5 \%)$ received oxygen therapy, including two patients who required invasive ventilator and one patient who required noninvasive ventilator. $92(46 \%)$ people had comorbidities: $52(26 \%)$ people had increased body weight, 28 (14\%) people had arterial hypertension, 7 (3.6\%) people had bronchial asthma, and $5(2.5 \%)$ people had type 2 diabetes. According to the results of the questionnaire, the most frequent disorders after having had a new coronavirus infection COVID-19 were: fatigability - 97 people $(48,5 \%)$, weakness - 77 people $(38,7 \%)$, sleep disorders - 58 people $(28,9 \%)$, memory disorders - 47 people $(23,7 \%)$, palpitations - 34 people $(17,2 \%)$.

Conclusion: Central and peripheral nervous system lesions were the most frequent symptoms after COVID-19 infection. Neurological disorders may be associated with neurotropicity and neurovirulence of SARS-CoV-2. damage to the central and peripheral nervous system due to "cytokine storm", hypoxemia due to respiratory disorders, microcirculatory disorders, homeostasis, Pathogenesis, clinical features, risk factors, as well as possibilities of correction of neurological disorders associated with COVID-19 require further study.

\footnotetext{
76

Defibrotide in the prevention and treatment of acute respiratory distress syndrome in patients with COVID-19. Preliminary safety results

MDPhD, PharmPhD Patricia Rodríguez-Fortúnez ${ }^{1}$ $\mathrm{PhD}$ Antonio J. Martínez-Mellado ${ }^{2}$, PhD Rubén Jara-Rubio ${ }^{2}$, $\mathrm{PhD}$ Miguel Blanquer-Blanquer ${ }^{2}, \mathrm{PhD}$ Pedro Castro-Rebollo ${ }^{3}$, $\mathrm{PhD}$ Andrés Carrillo-Alcaraz, ${ }^{4}$ PhD Consuelo Rodríguez-Jiménez $\mathbf{5}^{\mathbf{5}}$, $\mathrm{PhD}$ Helena Albendín ${ }^{2}, \mathrm{PhD}$ Elena Solano ${ }^{2}, \mathrm{PhD}$ Ana Pareja ${ }^{2}$,

$\mathrm{PhD}$ Jose J. García-Clavel ${ }^{6}, \mathrm{PhD}$ Jose M. Moraleda-Jiménez ${ }^{6}$

${ }^{1}$ Clinical Trials Unit. University Hospital of the Canary Islands. SCReN (Spanish Clinical Research Network) Platform, Santa Cruz de Tenerife, Spain

${ }^{2}$ University Clinical Hospital Virgen de la Arrixaca IMIB, Murcia, Spain

${ }^{3}$ Clínic Hospital, Barcelona, Spain
}

${ }^{4}$ University Hospital Morales Meseguer, Murcia, Spain

${ }^{5}$ University Hospital of the Canary Islands. La Laguna University, Santa Cruz de Tenerife, Spain

${ }^{6}$ Murcia University. University Clinical Hospital Virgen de la Arrixaca. IMIB, Murcia, Spain

Introduction: Currently, the search for treatments that demonstrate safety and efficacy in the treatment of SARS-CoV-2 infection is an unmet need. Defibrotide (DF), given its pleiotropic properties, including endothelial protection, anti-inflammatory and immunomodulatory activity, as well as anticoagulant and fibrinolytic activity, could become a therapeutic option for COVID-19 patients, particularly those with severe manifestations. Defibrotide has already been approved for veno-occlusive disease of the liver (VOD), which would facilitate its immediate access to patients.

Objectives: To evaluate the safety and efficacy of intravenous infusion of Defibrotide in the prevention and treatment of acute respiratory distress and cytokine release syndrome in patients with SARS-CoV-2 infection.

Methods: Prospective, multicentre, randomised, parallel, double-blind, placebo-controlled, prospective phase IIb clinical trial (CT). Defibrotide was infused as a 24-hour continuous intravenous infusion at a total dose of $25 \mathrm{mg} / \mathrm{kg} /$ day for 15 days. 150 patients will be randomized. Preliminary results from a planned interim safety analysis in the first 30 patients are presented. This CT has been approved by the Spanish Medicines Agency and the Ethics Committee of the Hospital Clínico Universitario Virgen de la Arrixaca. EudraCT No: 2020-001409-21. Clinicaltrials.gov: NCT04348383

Results: 30 patients with PCR-documented SARS-CoV-2 infection and pneumonia, with risk factors (IL-6 levels $>3$ normal levels) were recruited between April 2020 and September 2020. The mean age of the series was $58 \square 15$ years (range: $34-89$ years), $80 \%$ were male. Out of 30 patients, 24 $(80 \%)$ were grade $4-5$, and $6(20 \%)$ were grade 6 of the scale of 7 grades of the WHO.

We collected 161 adverse events (AE), four of which (2.48\%) were considered serious adverse events (SAEs) and the four patients who experienced them, died.

The reported SAEs are summarized as follows: 1 patient $(25 \%)$ had tracheal perforation with tracheo-oesophageal fistula, 1 patient $(25 \%)$ had acute pulmonary thromboembolism, 1 patient $(25 \%)$ had multiorgan dysfunction syndrome, 1 patient $(25 \%)$ had respiratory failure. None of the SAEs were considered to be related to the investigational product. In the interim analysis planned in the first 30 patients and performed by an external data safety monitoring committee (DSMC), no statistically significant differences were recorded between the control group and placebo in terms of occurrence of AEs and SAEs. These data were consistent with the known favourable safety profile of Defibrotide in VOD, and continuation of the trial was therefore recommended.

Conclusions: Based on the results obtained in this planned interim analysis, there is no evidence of safety risks associated with the use of Defibrotide in continuous intravenous infusion in patients with COVID19. None of the SAEs were considered to be related to the investigational product.

Acknowledgments: We are indebted to Maria Muñoz from the Clinical Trials Unit. Foundation for Health Training and Research of the Region of Murcia. Spain

68

Remdesivir-related adverse reactions in patients admitted for COVID-19 at Hospital Universitario de la Princesa Miss Diana María Campodónico ${ }^{1}$, Miss Antía Gómez Fernández ${ }^{1}$, Miss. Ana Casajús Rey ${ }^{1}$, Miss. Gina Mejía Abril ${ }^{1}$, Miss. Paola Águeda Camargo Mamani ${ }^{1}$, Dr. María Dolores Ochoa Mazarro ${ }^{1}$, Mr. Miguel Álvarez Montero ${ }^{1}$, Dr. Francisco Abad Santos ${ }^{1}$, Miss Azahara Lopez Raigada $^{1}$

1Hospital Universitario De La Princesa - Clinical Pharmacology department, Madrid, Spain 
Introduction: The coronavirus disease 2019 (COVID-19) pandemic is caused by severe acute respiratory syndrome coronavirus 2 (SARS-CoV2). Currently, the only antiviral drug with a licensed indication for COVID-19 treatment is remdesivir. It was approved or authorized for emergency use to treat this disease in around 50 countries.

Remdesivir, commercialized as Veklury, is a broad-spectrum antiviral indicated in patients over 12 years of age and weighting at least 40 kilograms with pneumonia requiring supplemental oxygen. It is under additional monitoring. Therefore, reporting any suspected adverse event or reaction is highly important in order to minimise any potential risks.

According to EMA assessment report, side effects in patients with COVID-19 may include liver inflammation, nausea, and an infusion-related reaction with nausea, low blood pressure, and sweating, among others.

Objectives: The main objective is to evaluate remdesivir's adverse events (AEs) in a cohort of COVID-19 patients admitted at the Hospital Universitario de La Princesa, who meet the criteria for its administration according to clinical practice.

Methods: Our project is a retrospective post-authorisation observational study. Data were collected from clinical histories of COVID-19 patients admitted at the Hospital Universitario de La Princesa and treated with remdesivir, from August 2020 to January 2021. In those patients suspected of AEs, it was applied the causality algorithm of the Spanish Pharmacovigilance System by two clinical pharmacologists separately. AEs classified as possible, probable or definite were selected.

Results: From a total population of 115 patients treated with remdesivir, data from 89 patients have been analyzed so far. $43 \%$ were women (38/ 89 ) and the average age was $64.71 \pm 14.12$ years. In terms of origin, $67 \%$ (60/89) of the patients were European, compared to 33\% Latin American. 24 suspected AEs were identified, of which 14 (16\% of patients) were confirmed as possible ( $86 \%)$, and probable (14\%). Most of the cases were males $(71 \%)$ and the elevation of transaminases was the most frequent $\mathrm{AE}$ identified $(78.5 \%)$. In addition, one case of hypotension related to drug infusion was detected, completely recovered after treatment withdrawal. Discussion and conclusions: The Spanish Pharmacovigilance System for Medicinal Products for Human Use is closely monitoring suspected AEs reported with COVID-19 treatments. According to the latest report in January 2021, 58 AEs cases with remdesivir have been reported. Most of them were in adult males and $74 \%$ were considered serious. Hepatobiliary disorders have been the most commonly reported, which are also referenced in the drug label as a very frequent alteration.

In contrast, COVID-19 disease, has also shown evidence of hepatic damage (incidence from 15 to $53 \%$ ), mainly manifested by an increase in transaminases accompanied by discrete elevations of bilirubin. This can also apply to other concomitant treatment.

The results of our study are consistent with data provided at national and European level, but according to the similarity between the analytical alterations inherent to the infection and those attributable to remdesivir, further studies and pharmacovigilance follow-up are necessary to determine these causalities in greater depth.

93

Prescription and adherence patterns of antithrombotic therapy for the secondary prevention of stroke in patients with atrial fibrillation Dr Francisco Sanchez-Saez ${ }^{1}$, Judit Riera-Arnau ${ }^{2}$,

${ }_{\text {Dr Isabel Hurtado-Navarro }}{ }^{1}$, Dr Clara Rodriguez-Bernal ${ }^{1}$,

Dr Gabriel Sanfélix-Gimeno ${ }^{1}$

${ }^{1}$ Foundation for the Promotion of Health and Biomedical Research of Valencia Region. FISABIO, Valencia, Spain

${ }^{2}$ Clinical Pharmacology Service, Vall d'Hebron University Hospital, Autonomous University of Barcelona, Barcelona, Spain

Introduction: Antithrombotic treatment (ATT) is recommended after stroke in patients with atrial fibrillation (AF). Therapy maintenance, from both physicians and patients, is a key aspect to avoid adverse clinical outcomes.

Objectives: To assess prescription (initiation, interruption and switching) and adherence patterns to therapeutic strategies used for the secondary prevention of stroke.

Methods: This retrospective, population-based observational study was conducted in the region of Valencia, Spain (5 million inhabitants). The cohort included all adult patients with AF or flutter hospitalized after an ischemic stroke or TIA from 2010 to 2015. This data was extracted from the Valencia Health System Integrated Database (VID). Exposure to three different ATT was considered: antiplatelet therapy (APT), oral anticoagulants (OAC), or both (OAC + APT). The initial treatment was defined as 30 consecutive days of ATT treatment (or 20 overlapping consecutive days when considering treatment with $\mathrm{OAC}+\mathrm{APT}$ ) within 60 days after hospital discharge from stroke or TIA. We calculated physician interruption of ATT and switching to an alternative antithrombotic strategy within 12 months. Interruption was defined as 60 consecutive days with no treatment and switching as 60 consecutive days with an ATT different from the index treatment (or at least 40 of these 60 days with both therapies for considering switching to OAC + APT). Long-term adherence was estimated through the proportion of days covered (PDC) at 12 months. Additionally, primary adherence was estimated for naïve patients.

Results: From 2010 to 2015, a total of 8084 patients with AF suffered a stroke or TIA. From these, $6521(80.7 \%)$ received ATT: APT (32\%), OAC $(49.3 \%)$, OAC + APT $(18.7 \%)$. The interruption of ATT by the physician after 1 year of follow-up was: $7.9 \%$ for APT, $8.3 \%$ for OAC and $6.2 \%$ for OAC+APT. Overall, $16.5 \%$ switch the antithrombotic strategy within the first year, $15.7 \%$ in the APT group, $3.6 \%$ in the OAC group, and $52.0 \%$ for patients on the combination strategy (the majority of those- $85 \%$-interrupted the APT and maintained the OAC therapy alone). Adherence to ATT at 12 months was $85 \%$, being highest for OAC $(90 \%)$. Primary adherence in new users was $94 \%$.

Conclusion: A high proportion of patients did not receive any antithrombotic therapy after having an ischemic stroke or TIA. After one year most of the treated cohort were still on treatment, while switching was not frequent with the exception of the group treated with the ATP and OAC combined, whom around half stopped the APT.

96

Interaction between Tacrolimus and Lopinavir/Ritonavir in transplant patients with COVID-19 and the role of CYP3A4 and immune response: Our experience

Dr David Valencia López ${ }^{1}$, Dr Noelia Vega Gil ${ }^{1}$,

Dr Rita Nogueiras Alvarez ${ }^{1}$, Dr Ivan Mazon Maraña ${ }^{1}$,

Dr Diana Zulema Cuellar Gómez ${ }^{1}$, Dr Andrea Bautista Blazquez ${ }^{1}$,

Dr Beatriz Alonso Gómez ${ }^{1}$, Dr M Angeles De Cos Cossío ${ }^{1}$,

Dr Mar García Saiz ${ }^{1}$, Dr Blanca Sánchez Santiago ${ }^{1}$

${ }^{1}$ Hospital Universitario Marqués De Valdecilla, Santander, Spain

Introduction: The COVID-19 pandemic has been a challenge for the medical community. In the absence of specific treatment, one of the drugs used was Kaletra ${ }^{\circledR}$ (lopinavir / ritonavir), which inhibits the activity of the CYP3A4 isoform of cytochrome P450. In transplant patients, monitoring Tacrolimus level is important to prevent toxicity due to that interaction. Objectives: To analyze Kaletra®-tacrolimus interaction in transplant patients admitted to hospital for COVID-19 and to study the influence of patients clinical situation and immune response on the magnitude of the interaction.

Methods: A review of transplant patients admitted to Hospital Marqués de Valdecilla for COVID-19 from March to June 2020 was performed. 
Blood tacrolimus levels were tested during Kaletra ${ }^{\circledR}$ therapy in our Clinical Pharmacology lab.

Results: Three out of 7 transplant patients admitted for COVID-19 received Kaletra ${ }^{\circ}$ :

A)65-year-old man with double lung transplant with previous outpatient stable level of tacrolimus and normal kidney-liver function; he stayed at ICU due to hemodynamic instability and kidney-liver impairment; IL6 and ferritin were high. On day 7, Tacrolimus level $>60 \mathrm{ug} / \mathrm{L}$ led to tacrolimus interruption. Despite the therapeutic measures applied, patient died. B)61-year-old woman and C)57-year-old man both kidney transplant recipients, both prior outpatient stable levels of Tacrolimus, stable impaired kidney function and normal liver. (B) did not show tacrolimus level variation despite dose was not adjusted. As preventive measure however, it was interrupted and level decreased below range. (C) dose was reduced by $50 \%$ and far from decreasing, level raised by $20 \%$, so tacrolimus was discontinued. Both $\mathrm{B}$ and $\mathrm{C}$ hemodynamic situation remained stable, and no renal nor hepatic impairment ocurred. IL6 and ferritin were normal in (B) and high in (C). At discharge they both returned to stable concentration.

Conclusions: 1: In case of hemodynamic instability, vasodilation and decreased blood flow leads to lower volumen of distribution of tacrolimus, increasing its blood level. 2: liver function impairment reduces drug elimination increasing blood concentration. 3: Greater Tacrolimus level elevation was observed in patients with high values of IL6 and ferritin, which is worth investigating in further studies.

97

Apixaban Therapy in a Cystic Fibrosis Patient Treated Chronically With Ivacaftor: Case Study

Dr. Ophir Lavon ${ }^{1,2}$, Dr. Michal Shteinberg ${ }^{2,3}$, Dr. Tal Lavi ${ }^{1}$

${ }^{\mathrm{C}}$ Clinical Pharmacology and Toxicology Unit, Carmel Medical Center, Haifa, Israel

${ }^{2}$ Rappaport Faculty of Medicine, Technion-Israel Institute of Technology, Haifa, Israel

${ }^{3}$ Pulmonology Institute and CF Center, Carmel Medical Center, Haifa, Israel

Introduction: Data regarding apixaban treatment in cystic fibrosis (CF) patients are limited. So, is the information on the interaction between apixaban and ivacaftor.

Objective: To present a case study of a CF patient treated with apixaban and ivacaftor.

Case study: A 33-year old male CF patient chronically treated with ivacaftor was admitted to the emergency department due to scant hemoptysis. Physical examination was unremarkable and laboratory results were normal including CRP and WBC count. COVID-19 PCR was negative. A $\mathrm{CT}$ angiography was performed and demonstrated perfusion defects in the superior branch of the right pulmonary artery as well as its subdivisions. A diagnosis of pulmonary embolism was made. The patient was started on low molecular weight heparin which was well tolerated. The hemoptysis resolved within 2 days. Treatment was switched to oral apixaban at a dose of $10 \mathrm{mg}$ bid for 7 days (loading dose), then changed to $5 \mathrm{mg}$ bid (maintenance dose). Due to concerns of potential drug interaction with ivacaftor, which has CYP3A4 inhibition properties, and due to limited data regarding apixaban pharmacokinetics in CF patients, we monitored drug levels, based on anti-fXa measurement. Peak level, 3 hours after administration of the first dose, was $334 \mathrm{ng} / \mathrm{ml}$, which verified appropriate absorption. Apixaban half-life, calculated based on drug levels within the first interval after the starting $10 \mathrm{mg}$ dose, was about 9 hours, which represents a relatively higher clearance than average. Steady state levels were evaluated after 3 days of treatment with $10 \mathrm{mg}$ bid and found to be within the reference ranges: $550 \mathrm{ng} / \mathrm{ml}$ (peak) and $274 \mathrm{ng} / \mathrm{ml}$ (trough). Steady state levels 3 days after reducing the dosing to $5 \mathrm{mg}$ bid were also within the reference ranges: 314 (peak) and 173 (trough). The patient continued the treatment without any further events.
Conclusion: Based on this case study, apixaban is well absorbed and eliminated in $\mathrm{CF}$ patients. Current dosing guidelines for apixaban are adequate in cystic fibrosis patients treated chronically with ivacaftor.

\section{Poster Session 3b - Safety-FV/Toxicology}

1

SAFETY RESULTS OF HEPATITIS B VACCINE ADSORBED AND ADJUVICATED BY AS04C (FENDRIX®) IN PATIENTS WHO DO NOT RESPOND TO VACCINATION

$\mathrm{MDPhD}$, PharmPhD Patricia Rodríguez-Fortúnez, PhD José L. Bravo-Grande ${ }^{2}$, PhD Ricardo López-Pérez ${ }^{3}$, Dr Carmen Arias-Fuente ${ }^{3}$, PhD Consuelo Rodríguez-Jiménez ${ }^{\mathbf{1 , 4}}$

${ }^{1}$ Clinical Trials Unit, Hospital Universitario De Canarias. SCReN (Spanish Clinical Research Network) Platform, Santa Cruz de Tenerife, Spain

${ }^{2}$ Occupational Risk Prevention Service, Complejo Asistencial Universitario de Salamanca., Salamanca, Spain

${ }^{3}$ Clinical Research and Clinical Trials Unit, Salamanca University Hospital - Salamanca Biomedical Research Institute (IBSAL) - SCReN (Spanish Clinical Research Network) Platform., Salamanca, Spain ${ }^{4}$ La Laguna University, Physical Medicine and Pharmacology Department, Santa Cruz de Tenerife, Spain

Introduction: Healthcare proffesionals are often exposed to biological risks. These include a risk of developing Hepatitis B virus infection. Therefore, there is a need to provide solutions for healthcare proffesionals where conventional vaccination has not been effective in order to offer them an alternative vaccine. Fendrix ${ }^{\circledR}$ is an adjuvant anti-hepatitis $B$ vaccine (rDNA), but its marketing authorisation restricts its use to the immunisation of patients with a lower immune response than healthy individuals.

Objectives: The aim of the study was to evaluate safety and effectiveness of the Fendrix ${ }^{\circledR}$ vaccine in healthcare proffesionals who do not respond to hepatitis $\mathrm{B}$ vaccination.

Methods: A non-commercial, open, multicenter clinical trial was performed at 11 Spanish health centers. This study was approved by the clinical research ethics committee of the Salamanca Healthcare Area and was identified with the Eudra-CT number: 2016-004991-23.

Results: 67 individuals were recruited between April 2018 and October 2019. The average age was $49.46 \pm 11.77$ years old and $30 \%$ were men. Throughout the trial, 32 adverse events (AEs) were collected, 25 of which were related to the study drug. All the AEs collected were considered non-severe: 31 mild and 1 moderate. All patients recovered from these events.

In detail, of the 32 AEs: 19 (59.38\%) corresponded to pain (in the area of the puncture), 4 cases $(12.5 \%)$ to discomfort, 3 cases $(9.38 \%)$ corresponded to fatigue, 2 cases $(6.25 \%)$ to diarrhea, 2 cases $(6.25 \%)$ to paresthesia, 1 case $(3.13 \%)$ to cramp and another was a case of diverticulitis. In summary, $78.12 \%$ of the AEs were related to the investigational drug. These AEs were transient and almost all resolved spontaneously within 1 week.

Conclusion: There is no evidence of potential risks related to the use of AS04C-added and adsorbed Hepatitis B Vaccine (Fendrix $\left.{ }^{\circledR}\right)$ in nonresponders to hepatitis $\mathrm{B}$ vaccination and who are at risk of infection due to their socio-occupational environment.

14

Patients' awareness on adverse effects of anxiolytic and hypnotic medications

$\mathrm{MD}, \mathrm{PhD}$, Assistant Professor Suzana Mimica Matanović ${ }^{1,2}$,

Dr Tara Cvijić ${ }^{1}$

${ }^{1}$ Faculty of Medicine, Josip Juraj Strossmayer University of Osijek, Osijek, Croatia 
${ }^{2}$ Unit for Clinical Pharmacology, Department of Internal Medicine, University hospital centre Osijek, Osijek, Croatia

INTRODUCTION: Anxiolytics and hipnotics belong to the most prescribed drugs in general. Most common indications for their use are generalized anxiety disorder, sleep disorders and panic disorder. Due high risk of abuse when using benzodiazepines, caution is needed on all aspects of their use.

OBJECTIVES: This cross - sectional study was conducted to assess patients' awareness of the adverse effects of anxiolytics and hypnotics and to evaluate patients' behavioral habits.

METHODS: Using an online survey, 80 adult male and female participants of different age groups were interwieved. Of the 80 subjects, 40 belonged to the control group not taking anxiolytics and hypnotics and the remaining 40 subjects took anxiolytics and hypnotics, either on-demand or continously. The enrolled participants were patients of three Family Medicine clinics from Osijek, Croatia.

RESULTS: Of the 40 participants currently taking anxiolytics and hypnotics, $58 \%$ were taking them on ,as needed“ basis. Diazepam was the most commonly used anxiolytic and was used by $48 \%$ of subjects. Alprazolam use was reported in $38 \%$ and zolpidem in $23 \%$ of subjects. Also, $68 \%$ of all participants confirmed that their main source of information on drug adverse effects was their physician. Alo, $79 \%$ percent of all participants were aware of the most important adverse reactions of anxiolytics and hypnotics (e.g. drowsiness, sedation, addiction potential) and $71 \%$ of them reported at least one adverse reaction. Ninety percent of participants taking these medications did not know which drugs are not recommended in combination with anxiolytics and hypnotics and $86 \%$ did not know how the adverse effects could be prevented. Of the total number of participants, $78 \%$ of participants confirmed that they regularly read the patient information leaflet and $65 \%$ was taking regularly their chronic therapy.

CONCLUSION: The participants in our survey, in both groups were sufficiently informed about the adverse effects of anxiolytics and hypnotics. There is still a need for education about the possible interactions of these drugs and methods to prevent adverse effects. Participants also showed adequate habits in most behavioral questions about pharmacotherapy.

\section{1}

\section{Unruly blood vessels - a fatal case of amlodipine poisoning} MD Henrik Magistad K. ${ }^{1}$, MD Lina Dorthea Bruun ${ }^{1}$, MD Sten Frøyshov

${ }^{1}$ Oslo University Hospital, Oslo, Norway

Introduction: Calcium channel blockers are widely used for a variety of indications - as such they are prescribed copiously both in primary care and hospitals. Amlodipine is a particularly popular CCB. The substance, consistently ranking in the top 10 most prescribed drugs the past 10 years, has been a mainstay in antihypertensive treatment in Norway. In 2019 it was the sixth most prescribed drug in defined daily doses (DDD). While not as toxic as its more cardioselective brethren verapamil and diltiazem, Amlodipine can still be a destructive force in severe overdoses.

Objectives: To present a case of fatal amlodipine poisoning and review and discuss the clinical picture, current treatment and preventative strategies.

Method: An elderly woman with a history of hypertension and recent cognitive decline was admitted to the Emergency Department. Her relatively lucid demeanour stood in stark contrast to a severely lowered blood pressure, O2-saturation and heart rate. Diagnostics revealed an accelerated nodal rhythm on ECG and an elevation of white blood cells and blood glucose, but otherwise unremarkable and inconclusive tests, including a high resolution CT-scan. A tentative diagnosis of cardiovascular-agent overdose was made, but despite apparent success of intensive care treatment the patient died two days later.

Results: A blood sample drawn on admittance showed a staggering amount of amlodipine in her system with an estimated serum concentration of $1500 \mathrm{nmol} / \mathrm{L}$. In the average well treated patient trough concentration of amlodipine are generally expected to be $<70 \mathrm{nmol} / \mathrm{L}$. Similarly, the amlodipine concentration in post-mortem peripheral blood was approximately $4300 \mathrm{nmol} / \mathrm{L}$ - several times higher than levels proposed as potentially lethal. Reported cause of death was aspiration pneumonia most likely set about by a massive amlodipine overdose.

Conclusion: Calcium channel blocker-toxicity can be lethal and extremely challenging to treat. Recognition of the clinical picture is important for early diagnosis. Successful treatment options have been reported from various cases, and rapid treatment initiation is key. Health care providers should be aware of the potential lethal consequence of a calcium channel blocker poisoning, ensuring safe administration both in hospital- and primary care.

27

Effect of corticosteroids in the outcome of drug-induced liver injury: a propensity score weighting analysis

Dr Hao Niu ${ }^{1}$, Dr Ismael Alvarez-Alvarez ${ }^{1,2}$, Dr Inmaculada Medina-Cáliz ${ }^{1}$, Ms Elvira Bonilla ${ }^{1}$, Mr Zeus Perez-Valdes ${ }^{1}$, Dr Mercedes Robles-Díaz ${ }^{2,3}$, Dr Miren García-Cortés ${ }^{2,3}$, Dr Judith Sanabria-Cabrera ${ }^{1,4}$, Dr Raul J Andrade ${ }^{2,3}$, Dr M Isabel Lucena ${ }^{\mathbf{1 , 3}, \mathbf{4}}$

${ }^{1}$ Servicio de Farmacología Clínica, Hospital Universitario Virgen de la Victoria, Instituto de Investigación Biomédica de Málaga-IBIMA, Universidad de Málaga, Málaga, Spain

${ }^{2}$ UGC Aparato Digestivo, Hospital Universitario Virgen de la Victoria, Instituto de Investigación Biomédica de Málaga-IBIMA, Universidad de Málaga, Málaga, Spain

${ }^{3}$ Biomedical Research Network Center for Hepatic and Digestive Diseases (CIBERehd), Carlos III Health Institute, Madrid, Spain

${ }^{4}$ Platform for Clinical Research and Clinical Trials IBIMA, Plataforma ISCiii de Investigación Clínica, Madrid, Spain

Introduction: To date, there is no specific therapy approved for idiosyncratic drug-induced liver injury (DILI) treatment. Corticosteroids are often empirically used in DILI patients although its benefit-risk balance remains undefined.

Objectives: To evaluate the effects of corticosteroids in DILI patients using non-experimental data through propensity score weighting (PSW) to balance the distribution of confounding factors and reduce their influence between the comparison groups.

Methods: All cases of idiosyncratic DILI entered in the Spanish DILI registry from 1994 to November 2020 were retrieved (N=979). The primary outcome was the development of acute liver failure (ALF) that went on to liver-related death/liver transplantation. Patients treated with corticosteroids were compared to those who did not receive any treatment. The inverse probability of treatment weighting with PS was used to reduce confounding due to age, sex, total bilirubin, alkaline phosphatase, type of liver injury and Model for End-stage Liver Disease score. Crude and adjusted odds ratio (OR) along with its $95 \%$ confidence interval $(\mathrm{CI})$ were estimated.

Results: A total of eligible 548 patients, 78 treated with corticosteroids, 2 of them also treated with ursodeoxycholic acid, and 1 with ursodeoxycholic acid and MARS (mean age $54 \pm 18$ years, $58 \%$ women), and 470 who did not receive any treatment ( $54 \pm 18$ years; $48 \%$ women), were enrolled. Hepatocellular injury was more frequent in both groups (63\% and $66 \%$, respectively). Among patients who received corticosteroids $6.4 \%$ developed ALF, compared to $1.9 \%$ who did not receive any treatment $(\mathrm{p}=0.036)$, and risk of ALF was significantly increased in patients who received corticosteroids (crude OR=3.50; 95\% CI 1.14-10.76, $\mathrm{p}=0.028$ ). After PSW, 363 patients were included in 2 well-balanced 
groups. Corticosteroids use was not associated with an increased risk for developing ALF (adjusted OR=2.28; 95\% CI 0.66-7.88; $\mathrm{p}=0.194$ ). Sensitivity analysis excluding drug-induced autoimmune hepatitis cases $(\mathrm{N}=29)$ showed consistent findings (adjusted $\mathrm{OR}=3.66$; 95\% CI 0.85 $15.82, \mathrm{p}=0.083$ ).

Conclusion: This PSW study evidenced that corticosteroids administration in DILI patients was not associated with an increased risk of progression to ALF in DILI patients. Further collaborative studies to validate these findings are clearly needed.

\section{7}

Is Levomecol (Chloramphenicol+Dioxomethyltetrahydropyrimidine) so safe? (Adverse reaction to Chloramphenicol. Clinical case) Albina Nigmedzyanova ${ }^{1,2}$, Albina Zvegintseva ${ }^{1,2}$, Ludmila Kulagina ${ }^{1,2}$, Maxim Maksimov $^{1,2}$, Ramilya Gatina ${ }^{1}$, Elvina Kadiseva ${ }^{1}$

${ }^{1}$ Republican Clinical Hospital Ministry of health Republic of Tatarstan, Kazan, Russian Federation

${ }^{2}$ Kazan State Medical Academy - Branch Campus of the Federal State Budgetary Educational Institution of Further Professional Education «Russian Medical Academy of Continuous Professional Education» of the Ministry of Healthcare of the Russian Federation, Kazan, Russian Federation

Introduction: Despite the fact that chloramphenicol is recommended as a reserve drug for the treatment of severe infections, in which less toxic antibacterial agents are ineffective or contraindicated, in clinical practice it is often used for the treatment of purulent inflammatory diseases of soft tissues.

Objective: to analyze a clinical case of acute myeloblastic leukemia, first detected, myelotoxic agranulocytosis, mild thrombocytopenia, mild anemia due to the combined preparation Levomecol (Chloramphenicol+ Dioxomethyltetrahydropyrimidine).

Materials and methods: Patient D., 53 years old was in the hematology department with the diagnosis "Acute myeloblastic leukemia first detected. Myelotoxic agranulocytosis. Mild thrombocytopenia. Anemia of mild degree. trigeminal neuritis." In the anamnesis, since 2017, the patient was suffering from headache, sore throat, frequent sore throat, left ear pain, rash, appearance of spontaneous bruises. During the last 2 years, she used Levomecol ointment uncontrollably to treat skin problems. On examination - petechial rash all over the body. Blood tests on the 1st day of hospitalization: leukopenia $(0.7 * 109 / 1)$, agranulocytosis, neutropenia $(0 \%)$, thrombocytopenia $(40 * 109 / 1)$, anemia (red blood cells$3.00 * 1012 / 1$, hemoglobin-94 $\mathrm{g} / 1$, hematocrit $28.1 \%$; iron $32.5 \mu \mathrm{mol} / \mathrm{l}$ ). Myelogram: myelocaryocytes 126000 , megakaryocytes 7.4 , blasts $76.5 \%$. Cytogenetic study revealed from two to several chromatid breaks of different chromosomes in $75 \%$ of cells. Treatment - polychemotherapy on the scheme $7+3$ (cytarabine and idorubicin), allopurinol, omeprazole up to 23 days with a negligible effect. On the 23rd day of treatment, selfadministration of levomecol was detected, the drug was banned.

Results: After withdrawal of the drug, clinical and laboratory improvement began - rash disappeared, frequency and intensity of headaches decreased, improvement of blood parameters was observed. Discharged on the 31st day of hospitalization with the diagnosis: Acute myeloblastic leukemia first detected. Condition after 1 course of induction according to the $7+3$ scheme. Myelotoxic agranulocytosis. Mild thrombocytopenia. Mild anemia...type:4560,XX,chtb(1)(p32),chtb(2)(p21),chtb(3)(p12),chtb(3)(p21),chtb(5)(p1?), chtb(6)(q12), chtb(6)(p21), chtb(6)(q2?5), chtb(8)(q22), chtb(10)(p13),-11,$12,-13,-19,-20,+3 \sim 20 \operatorname{mar}[\mathrm{cp} 15] / 46, \mathrm{XX}$ [5]. Blood parameters at discharge: leukopenia $(1.0 * 109 / 1)$, agranulocytosis, neutropenia (10.8\%), thrombocytopenia $(238 * 109 / 1)$, anemia (erythrocytes-2.89*1012/1, hemoglobin-80 g/l, hematocrit $26.0 \%$ ).

At the second course of chemotherapy 18 days after her admission her blood count was $2.8 * 109 / 1,43.9 \%$ neutrophils, $231 * 109 / 1$ platelets, anemia (erythrocytes-3.27*1012/1, hemoglobin-100 g/l, hematocrit $32.3 \%$ ). Myelogram: myelocaryocytes 64666 , megakaryocytes 14.81 , blasts $3.0 \%$

Conclusion: According to the instructions, Chloramphenicol in any form has a dose-dependent reversible bone marrow suppression due to suppression of mitochondrial protein synthesis in bone marrow cells. Undesirable drug reactions are manifested as toxic effects on bone marrow, blood changes (leukopenia, thrombocytopenia, neutropenia), hypoplastic anemia (occurs after several weeks or months), fever, bleeding, rash, headache, peripheral neuritis, sore throat, etc., which occurred in our patient. Improvement of the condition against the background of levomecol withdrawal confirms the assumption about the role of levomecol in the debut and the course of the underlying disease.

58

Rituximab-induced serum-sickness (RISS) in lymphoma Almudena Ramirez-Garcia ${ }^{1}$, Arantxa Sancho-Lopez ${ }^{1}$, Almudena De Laiglesia ${ }^{2}$, Antonio Caballero-Bermejo, Elena Diago-Sempere ${ }^{1}$, Concepción Payares-Herrera ${ }^{1}$, Laura Javaloyes-Garachana ${ }^{1}$, Cristina Avendaño-Solá ${ }^{1}$, Belén Navarro ${ }^{2}$

${ }^{1}$ Clinical Pharmacology, Universitary Hospital Puerta De Hierro Majadahonda

${ }^{2}$ Haematology, Universitary Hospital Puerta De Hierro Majadahonda, Spain

Introduction: Rituximab is a humanized murine antibody used in the treatment of B-cell lymphomas and has been very rarely associated with serum sickness (SS), a type-3 hypersensitivity reaction consisting of immune complexes deposition due to anti-drug antibodies (ADA) in different organs with a potentially severe inflammatory reaction.

Objectives: We describe a case of serum sickness in a patient receiving rituximab for nodal marginal zone lymphoma

Methods: A 69-year-old man was treated with rituximab biosimilar plus bendamustin. During the third cycle, the patient developed generalized and invalidating symmetric joint pain along with malaise, fatigue, feverishness, jaundice, nausea and diarrhoea, and was hospitalised.Blood tests showed hyperbilirubinemia, lymphopenia, thrombocytopenia and elevated CRP. Additional serological and laboratory tests excluded infectious polyarthritis and immune mediated rheumatic conditions. The diagnosis of RISS was suspected based on compatible clinical presentation and chronology of RTX administration.

Results: The patient was tested for serum ADA, with high levels of serum anti-RTX antibodies on day 35 since last RTX administration (4316 UA/ $\mathrm{mL}$ ) with undetectable RTX titres. It was advised not to reintroduce rituximab therapy but to consider alternative treatment with ofatumumab (fully-human) or obinutuzumab (humanized) anti CD20 antibodies. RISS is a different clinical entity to the commonly reported cytokine release infusion-reactions. Clinicians must consider it and avoid further infusions in patients who develop fever or arthralgia and elevated inflammatory parameters within 10 days after administration. ADA testing should be considered if suspected RISS but a single negative determination cannot discard diagnosis due to potentially inhibited humoral responses by chemotherapy and high consumption by excessive amounts of antigen (RTX) in the acute phase. The risk of developing RISS has been shown to be much higher with underlying autoimmune conditions; it would be advisable to rule out these in any patient with RISS.

Conclusion: SS was not reported in clinical trials and it is believed to be extremely rare in hematological malignancies. To our knowledge, this is the fourth case of RISS reported in a hematologic patient with no autoimmune condition. We believe it is relevant to increase awareness to enhance clinical suspicion, diagnosis and appropriate management. 
73

RISK OF INFECTIONS AMONG JAK INHIBITORS USED IN RHEUMATOID ARTHRITIS: A NETWORK META-ANALYSIS OF CLINICAL TRIALS

Dr. Ana Penedones ${ }^{1}$, Prof. Carlos Alves ${ }^{1,2}$, Dr. Diogo Mendes ${ }^{1}$, Prof. Francisco Batel-Marques ${ }^{1,2}$

${ }^{1}$ CHAD - Centre for Health Technology Assessment and Drug Research, AIBILI, Coimbra, Portugal

${ }^{2}$ Laboratory of Social Pharmacy and Public Health, School of Pharmacy, University of Coimbra, Coimbra, Portugal

Introduction: The Janus kinases (JAKs) are cytoplasmic tyrosine kinases associated with membrane cytokine receptors that mediate signalling of multiple cytokines and growth factors, contributing to the pathogenesis of multiple autoimmune disorders. JAK inhibitors are a new class of targeted therapies that improve the symptoms of rheumatoid arthritis, but have been associated with an increased risk of infections.

Objectives: This study is aimed at assessing the risk of infections associated with JAK inhibitors in patients with rheumatoid arthritis.

Methods: PUBMED, EMBASE, Cochrane Library and clinicaltrials.gov were searched to identify randomized controlled trials (RCTs) evaluating the efficacy and safety of JAK inhibitors in patients with rheumatoid arthritis. The outcomes assessed were the risk of infections, namely tuberculosis and herpes zoster. Network meta-analyses were performed using STATA 13.1.. For each outcome, treatments were ranked according to the probability of being the safest (best) alternative using the surface under the cumulative ranking curve (SUCRA). A sensitivity analysis disaggregating the results according to background therapy and JAK inhibitors licensed doses. Results: Thirty-seven RCTs were included in the network meta-analysis. No differences were observed between the treatments regarding the risk of tuberculosis. According to SUCRA ranking for the risk of tuberculosis, baricitinib is probably the safest treatment (SUCRA =0.807), followed by methotrexate (SUCRA=0.561) and upadacitinib (SUCRA=0.495). Compared to filgotinib, adalimumab (4.81; 95\% CI 1.39 - 16.66), etanercept (6.04; 95\% CI 1.79 - 20.37), peficitinib $(7.56 ; 95 \%$ CI 1.63 - 35.12), tofacitinib $(4.29 ; 95 \% \mathrm{CI}$ $1.43-12.88)$ and upadacitinib $(4.35 ; 95 \%$ CI 1.46 - 13.00) have an increased risk of herpes zoster infection. SUCRA ranking for the risk of herpes zoster suggests that filgotinib is probably the safest treatment $(\mathrm{SUCRA}=0.968)$, followed by placebo $(\mathrm{SUCRA}=0.726$ ) and baricitinib (SUCRA=0.619). Risk differences between the drugs became statistically non-significant when the sensitivity analysis was conducted.

Conclusion: The initial results suggest that filgotinib has the lower risk of herpes zoster infection among the drugs evaluated. However, the sensitivity analyses did not support those findings. Evidence also suggests that the risk of tuberculosis among JAK inhibitors may be comparable.

\section{4}

RISK OF CARDIOVASCULAR AND VENOUS THROMBOEMBOLISM EVENTS AMONG JAK INHIBITORS USED IN RHEUMATOID ARTHRITIS: A NETWORK META-ANALYSIS OF CLINICAL TRIALS

Dr. Ana Penedones ${ }^{1}$, Prof. Carlos Alves ${ }^{1,2}$, Dr. Diogo Mendes ${ }^{1}$, Prof. Francisco Batel-Marques ${ }^{1,2}$

${ }^{1}$ CHAD - Centre for Health Technology Assessment and Drug Research, AIBILI, Coimbra, Portugal

${ }^{2}$ Laboratory of Social Pharmacy and Public Health, School of Pharmacy, University of Coimbra, Coimbra, Portugal

Introduction: Janus kinases (JAK) inhibitors are a new class of targeted therapies that demonstrated to be effective in the treatment of adult patients with moderate to severe active rheumatoid arthritis. However, the incidence of thromboembolic events (VTEs) in patients treated with JAK inhibitors during clinical trials (RCTs) led regulatory authorities to recommend special precautions and further investigation.

Objectives: This study is aimed at assessing the risk of Major Adverse Cardiovascular Events (MACE) and VTE associated with JAK inhibitors in patients with rheumatoid arthritis.

Methods: PUBMED, EMBASE, Cochrane Library and clinicaltrials.gov were searched to identify RCTs evaluating the efficacy and safety of JAK inhibitors in patients with rheumatoid arthritis. The outcomes assessed were the risk of MACE (myocardial infarction, cerebrovascular accident [ischaemic and haemorrhagic strokes] or cardiovascular death) and VTE (pulmonary embolism and deep vein thrombosis). For each outcome, treatments were ranked according to the probability of being the safest (best) alternative using the surface under the cumulative ranking curve (SUCRA). A sensitivity analysis disaggregating the results according to background therapy and JAK inhibitors licensed doses.

Results: Thirty-seven RCTs were included in the network meta-analysis. No differences were observed between the treatments regarding the risk of MACE. The ranking probability based on the SUCRA suggests that filgotinib is probably the safest treatment regarding MACE occurrence (SUCRA=0.667), followed by tofacitinib (SUCRA=0.660) and baricitinib (SUCRA=0.622). Tofacitinib has a reduced risk of VTE when compared to placebo $(0.33$; 95\% CI 0.12 $0.93)$. No further statistically significant VTE risk differences were observed. The ranking probability based on the SUCRA suggests that peficitinib is probably the safest treatment regarding VTE occurrence (SUCRA=0.786), followed by tofacitinib (SUCRA=0.719) and filgotinib (SUCRA=0.620). Risk estimates of both outcomes did not significantly change from the initial results after conducting the sensitivity analysis.

Conclusion: The results suggest that JAK inhibitors present similar risks of both MACE and VTE. Postmarketing pharmacovigilance data will be of utmost importance to confirm these findings.

\section{0}

Adverse drug reactions of cannabis and cannabinoids reported in the international pharmacovigilance database Vigibase ${ }^{\circledR}$

Doctor Emilie Jouanjus ${ }^{1}$, Ms Amélia Déguilhem², Ms Sonia Merad ${ }^{3}$, Doctor Maryse Lapeyre-Mestre ${ }^{4}$

${ }^{1}$ Addictovigilance Center, Toulouse University Hospital; CERPOP, UMR INSERM-University of Toulouse, Toulouse, France

${ }^{2}$ AP-HP, Paris Public Hospitals, Paris, France

${ }^{3}$ CERPOP, UMR INSERM-University of Toulouse, Toulouse, France

${ }^{4}$ Addictovigilance Center, Toulouse University Hospital; Clinical Investigation Center 1436, Toulouse University Hospital, Toulouse, France

Introduction: Data on the potential risks associated with the use of cannabis or cannabinoid derivatives are a cause for concern. The objective was to describe the reports of adverse drug reactions (ADRs) related to the use of cannabis and its derivatives, by analyzing the individual case safety reports (ICSRs) recorded in Vigibase ${ }^{\circledR}$.

Methods: VigiBase ${ }^{\circledR}$ is the World Health Organization (WHO) global database of ICSRs and the largest international pharmacovigilance database. We identified ADRs of the Cannabis sativa plant and its natural or synthetic derivatives by extracting the corresponding ICSRs recorded in VigiBase ${ }^{\circledR}$ between 01/01/2010 and 31/12/2019. ICSRs were eligible when: i) the patients' sex and age were mentioned in patients older than two years old, ii) at least one MedDRA term of any system organ class (SOC) was associated, and iii) provided that cannabis or at least one of its derivatives were recorded as "suspect".

Results: Among 10,867,883 records matching our inclusion criteria in Vigibase ${ }^{\circledR}$ in 2010-2019, 3,656 ICSRs were reported with at least one suspect mention of cannabis or derivatives, corresponding to 12,825 ADRs. Most ICSRs were serious (2,453, 67,1\%). Except Morocco (74.5 
per 10,000), the countries which recorded the highest numbers of these "cannabis ICSRs" relative to the total number of national ICSRs were Canada (16.3 per 10,000), France (12.7), Australia (9.6) and Italy (9.1). The concerned subjects were 37 years old on average and more frequently men $(2,145$ ICSRs, $58,7 \%)$. The primary drug was Cannabis sativa (2,384 mentions), followed by the delta-9-tetrahydrocannabinol (THC): cannabidiol (CBD) combination (775), dronabinol (407) and cannabidiol (310). The most frequent ADRs were psychiatric system disorders (29.7\%), the majority of which was related to Cannabis sativa. Nervous system disorders (13.0\%) was the most frequent SOC of CBD (17.1\%), THC:CBD (21.6\%) and nabilone (19.3\%). Gastrointestinal, cardiovascular and respiratory disorders represented $7.2 \%, 5.3 \%$ and $4.0 \%$ of ADRs. Conclusion: This extensive analysis of ADRs related to cannabis and its derivatives are contributive to an enhanced knowledge of the safety profile of the concerned drugs and could prove useful for professionals involved in the support and care for patients exposed to these drugs.

\section{S3- COVID drug repurposing}

81

Tocilizumab RCTs for COVID19. Absence of evidence is not evidence of absence

MD Antonio Caballero Bermejo ${ }^{1}$, MD Elena Diago Sempere ${ }^{1}$,

MD Almudena Ramirez Garcia ${ }^{1}$,

MD, PhD Concepcion Payares Herrera ${ }^{1}$,

MD, PdH Gustavo Adolfo Centeno Soto ${ }^{1}$,

MD Laura Javaloyes Garachana ${ }^{1}$, MD, PhD Aranzazu Sancho Lopez ${ }^{1}$, $\mathrm{MD}, \mathrm{PhD}$ Belen Ruiz Antoran ${ }^{1}, \mathrm{MD}, \mathrm{PhD}$ Cristina Avendaño Sola ${ }^{1}$

${ }^{1}$ Clinical Pharmacology, Hospital Universitario Puerta De Hierro Majadahonda, Madrid, Spain

Introduction: Tocilizumab is a recombinant monoclonal antibody against IL-6 and currently under investigation for treatment of patients with COVID19. Several observational studies $(1,2)$ and randomized clinical trials(RCT) (3-8) have evaluated efficacy of tocilizumab in COVID19 patients. Conflicting results from these studies have tempered initial enthusiasm for its use and caused divergences in treatment recommendations (9). Objectives: The overall objective is to compare patients' characteristics and trial design of reported tocilizumab RCT in order to identify potential explanations for divergent results.

Methods: A systematic review of online databases Pubmed and medRxiv was conducted (up to February 12, 2021) using the following search terms: 'tocilizumab', 'COVID-19' and 'randomized clinical trial'.

Results: Eight RCTs have been published to date. Baseline comorbidities, age and gender were similar in these studies. Differences are seen in baseline severity of disease and concomitant medication. More than $80 \%$ of patients recruited in the only 2 RCT in which the threshold for efficacy was actually met also received systemic corticosteroid therapy. Also those trials included patients with more advanced stages of COVID19 and have a larger sample size. Studies with negative results were generally underpowered and have performed sample size estimations with overestimation of the worsening rate in control group and in magnitude of effect (mortality day 28 estimated for sample size determination of $30-50 \%$ vs actual mortality in clinical trials of $12-20 \%$ ).

Conclusions: Tocilizumab has shown clinical benefit in both REMAP-CAP and RECOVERY trials. The perception of conflicting results is heavily influenced by a number of underpowered RCT which assumed worse rates of mortality than the actual ones. In a situation of uncertainty and rapid improvement of patient management, response-adaptive design is a good option to achieve an adequate sample size. Initiatives to facilitate multicenter and collaborative clinical trials are also acutely needed in most EU countries.

1. Ruiz-Antorán B. Combination of Tocilizumab and Steroids to Improve Mortality in Patients with Severe COVID-19 Infection: A Spanish,
Multicenter, Cohort Study. Infect Dis Ther. December 2020. doi:https:// doi.org/10.1007/s40121-020-00373-8

2. Rodríguez-Baño J. Treatment with tocilizumab or corticosteroids for COVID-19 patients with hyperinflammatory state: a multicentre cohort study (SAM-COVID-19). Clin Microbiol Infect. August 2020. doi:https://doi.org/10.1016/j.cmi.2020.08.010

3. Salvarani C. Effect of Tocilizumab vs Standard Care on Clinical Worsening in Patients Hospitalized With COVID-19 Pneumonia. JAMA Intern Med. 2021;181(1):24. doi:https://doi.org/10.1001/ jamainternmed.2020.6615

4. Veiga VC. Effect of tocilizumab on clinical outcomes at 15 days in patients with severe or critical coronavirus disease 2019: randomised controlled trial. BMJ. January 2021:n84. doi:https://doi.org/10.1136/bmj.n84 5. Hermine O. Effect of Tocilizumab vs Usual Care in Adults Hospitalized With COVID-19 and Moderate or Severe Pneumonia. JAMA Intern Med. 2021;181(1):32. doi:https://doi.org/10.1001/ jamainternmed.2020.6820

6. Stone JH. Efficacy of Tocilizumab in Patients Hospitalized with Covid-19. N Engl J Med. October 2020:NEJMoa2028836. doi:https://doi.org/10.1056/ NEJMoa2028836

7. Salama C. Tocilizumab in Patients Hospitalized with Covid-19 Pneumonia. N Engl J Med. 2021;384(1):20-30. doi:https://doi.org/10. 1056/NEJMoa2030340

8. Rosas IO. Preprint COVACTA. Tocilizumab in Hospitalized Patients With COVID-19 Pneumonia. https://www.medrxiv.org/content/10.1101/ 2020.08.27.20183442v1.full.pdf. Published 2020.

9. McCreary EK. Covid-19 controversies: the tocilizumab chapter. BMJ. January 2021:n244. doi:https://doi.org/10.1136/bmj.n244

84

Potential value of CRP, D-dimer, IL6 and lymphopenia as predictors for tocilizumab efficacy in COVID-19

MD Antonio Caballero Bermejo ${ }^{\mathbf{1}}$, MD, PhD Gustavo Adolfo Centeno Soto ${ }^{1}$, MD Elena Diago Sempere ${ }^{\top}$, MD Almudena Ramirez Garcia ${ }^{1}$, MD, PhD Concepcion Payares Herrera ${ }^{1}$, MD Laura Javaloyes Garachana ${ }^{1}, \mathrm{MD}, \mathrm{PhD}$ Ana Fernandez Cruz ${ }^{2}, \mathrm{MD}, \mathrm{PhD}$ Belen Ruiz Antoran ${ }^{1}, \mathrm{MD}, \mathrm{PhD}$ Aranzazu Sancho Lopez ${ }^{1}$

${ }^{1}$ Clinical Pharmacology, Hospital Universitario Puerta De Hierro Majadahonda, Madrid, España

${ }^{2}$ Infectious Diseases Unit, Internal Medicine Department, Hospital Universitario Puerta De Hierro Majadahonda, Madrid, España

Introduction A systemic inflammatory response is observed in coronavirus disease 2019 (COVID-19) (1). Severe COVID-19 cases are marked with excess cytokine production and a higher mortality rate $(2,3)$. Several biomolecules have been explored as possible biomarkers for outcome and treatment response $(4,5)$.

Objectives: We aimed to explore associations between CRP, D-dimer, lymphopenia and IL6 levels and magnitude of tocilizumab effect on clinical outcomes in patients with severe COVID-19.

Methods: We performed a multicentre retrospective cohort study in 18 tertiary hospitals in Spain from March to April 2020. Eligible patients were hospitalised patients with documented pneumonia and severe but non-critical respiratory failure: Brescia-COVID Scale score of 2, not in Intensive Care Unit. Of these, patients who received tocilizumab therapy according to clinical practice were assigned to the tocilizumab cohort, whilst patients who did not were assigned to the control cohort.

Results: During the study period, 506 patients with severe COVID-19 fulfilled inclusion criteria. Among them, 268 were treated with tocilizumab and 238 patients were not. Global mortality was $23.7 \%$. Mortality was lower in patients treated with tocilizumab than in controls (16.8\% versus $31.5 \%$, HR 0.51 (95CI 0.35-0.744), p<0.001) (6). Patients with greater elevation of CRP have a positive effect with tocilizumab treatment. In the group of patients with CRP> $75 \mathrm{mg} / 1$ the mortality in patients treated with tocilizumab was lower than in non-treated patients 
(16.3\% versus $34.9 \%$, HR 0.39 (95CI 0.25-0.5), while tocilizumab effect was not evident for subgroup with CRP $<75 \mathrm{mg} / 1 ; 18.1 \%$ vs $22.7 \% \mathrm{HR}$ 0.63 (95CI $0.30-1.34$ ). No differences were found for the rest of the biomarkers.

Conclusions: In hospitalised COVID-19 patients with hypoxia and systemic inflammation, tocilizumab improved survival. CRP elevation could be a good predictor for response to tocilizumab in COVID19 patients.

\section{References}

1. Cron RQ. COVID-19 cytokine storm: targeting the appropriate cytokine. Lancet Rheumatol. February 2021. doi:10.1016/S26659913(21)00011-4

2. Fernández-Cruz A, Ruiz-Antorán B, Muñoz-Gómez A, et al. A Retrospective Controlled Cohort Study of the Impact of Glucocorticoid Treatment in SARS-CoV-2 Infection Mortality. Antimicrob Agents Chemother. 2020;64(9). doi:10.1128/AAC.01168-20

3. Fernández-Ruiz M, López-Medrano F, Pérez-Jacoiste Asín MA, et al. Tocilizumab for the treatment of adult patients with severe COVID-19 pneumonia: A single-center cohort study. J Med Virol. 2021;93(2):831842. doi: $10.1002 / \mathrm{jmv} .26308$

4. Sahu BR, Kampa RK, Padhi A, Panda AK. C-reactive protein: A promising biomarker for poor prognosis in COVID-19 infection. Clin Chim Acta. 2020;509:91-94. doi:10.1016/j.cca.2020.06.013

5. Rahi MS, Jindal V, Reyes S-P, Gunasekaran K, Gupta R, Jaiyesimi I. Hematologic disorders associated with COVID-19: a review. Ann Hematol. 2021;100(2):309-320. doi:10.1007/s00277-020-04366-y

6. Ruiz-Antorán B, Sancho-López A, Torres F, et al. Combination of Tocilizumab and Steroids to Improve Mortality in Patients with Severe COVID-19 Infection: A Spanish, Multicenter, Cohort Study. Infect Dis Ther. December 2020. doi:10.1007/s40121-020-00373-8

Poster Session 4a - Medication errors/drug utilization/prescription/ education

44

Increasing pharmacotherapy knowledge and skills in medical students by practicing with e-learning program $\mathrm{P}$-scribe

Msc Laura Kalfsvel ${ }^{1}$, Dr. Jorie Versmissen ${ }^{1}$, Adriaan van Doorn ${ }^{2}$, Prof. Dr. Walter van den Broek ${ }^{1}$, Prof. Dr. Hugo van der Kuy ${ }^{1}$, Dr. Floor van Rosse ${ }^{1}$

${ }^{1}$ Erasmus Mc, University Medical Center, Rotterdam, the Netherlands

${ }^{2}$ University Medical Center Groningen, Groningen, the Netherlands

Introduction: Junior doctors write most hospital prescriptions, yet are more than twice as likely to make an error in their prescriptions compared to medical consultants. A possibility to enhance pharmacotherapy education is through the use of e-learning modules.

Objectives: The aim was to determine whether P-scribe, as the chosen e-learning resource, helps students in their development of pharmacotherapy.

Methods: This retrospective study was undertaken in the Erasmus Medical Center, the Netherlands. All 270 medical students who started their master curriculum in the academic session of 2017-2018 were included. Data were analyzed to identify the frequency of student's use per e-learning module, total time students spent on e-learning modules and pattern of the use of e-learning modules in relation to their assessments. The results on assessments were analyzed to identify possible correlations between the time students spent using P-scribe, their patterns of use and their assessment results.

Results: Students who passed their knowledge-based assessment at once had a mean practice time of five more hours than students who did not pass at once $(\mathrm{P}<0.05)$. These students practiced on average six e-learning modules more $(\mathrm{P}<0.05)$ than students who failed their first attempt. Students who passed their skill-based prescription test at once, practiced on average five more e-learning modules $(\mathrm{P}=0.006)$ than students who failed their first attempt.

Conclusion: Students who passed their pharmacotherapy assessments at once spent more time, and practiced more frequently, with e-learning modules. E-learning modules should therefore offer students the possibility to practice frequently throughout the whole curriculum.

47

Real World Evaluation of the IV Iron Service at Alrijne Hospital Group

Dr Roel Fijn ${ }^{1}$, Ir. Hans C. Ablij ${ }^{2}$, Dr. Pieter D. Knoester ${ }^{1}$,

Dr. Annelies W.E. Weverling-Rynsburger ${ }^{2}$, Dr. Anne M.C. Witte ${ }^{3}$

${ }^{1}$ Alrijne Hospital Group, Department of Clinical Pharmacy, Leiden, The Netherlands

${ }^{2}$ Alrijne Hospital Group, Department of Internal Medicine, Leiden, The Netherlands

${ }^{3}$ Alrijne Hospital Group, Department of Gastroenterology, Leiden, The Netherlands

Introduction: Iron deficiency anaemia is common in a broad range of conditions including cases of blood loss or chronic inflammatory diseases, where oral iron absorption is impaired. Therefore, intravenous iron (IV) is increasingly being used in such patients when oral iron is not efficacious/ well-tolerated or there is a need for rapid iron delivery. An IV iron service using ferric derisomaltose (FDI) is well-established in Alrijne Hospital Group.

Objective: To evaluate the real-world effectiveness of the IV iron service. Methods: The medical records of patients receiving FDI between JanDec 2019 were retrospectively reviewed. Patients referred from gynaecology or undergoing haemodialysis were excluded from the analysis. Data on demographics, haemoglobin $(\mathrm{Hb})$, iron dose and adverse drug reactions (ADRs) were extracted and analysed. Actual iron dose given was compared to the iron dose according to the FDI SmPC. Change in $\mathrm{Hb}$ from baseline to 4-12 weeks post-infusion was the main efficacy outcome. Safety was evaluated by reviewing reported ADRs.

Results: A total of 433 patients received 604 FDI infusions in the audit period. Referrals included patients from internal medicine $(57 \%)$, gastroenterology $(26 \%)$, cardiology $(4 \%)$, geriatrics $(4 \%)$, respiratory medicine $(4 \%)$ and other departments $(5 \%)$. Mean age was 68 years, weight $76 \mathrm{~kg}$, and $247(57 \%)$ patients were women. Baseline and follow-up $\mathrm{Hb}$ data were available for 257 (43\%) infusions. Mean FDI dose was $1269 \mathrm{mg} ; 60 \%$ of patients with available data were underdosed. Mean $\mathrm{Hb}$ rose from $97 \mathrm{~g} / \mathrm{L}$ at baseline to 116 $\mathrm{g} / \mathrm{L}$ at follow-up, where the proportion of patients with an $\mathrm{Hb}$ increase $\geq 20 \mathrm{~g} / \mathrm{L}$ was $56 \%$ and $55 \%$ for patients dosed correctly or underdosed, respectively. A greater proportion of patients in the correctly-dosed group reached non-anaemic status (41\% vs. $25 \%$ ). ADRs were observed in 19 (3\%) of administrations; 18 ADRs were non-serious and the infusion was completed successfully, while 1 $(0.16 \%)$ case was considered serious.

Conclusion: Follow-up $\mathrm{Hb}$ data were not available for a large proportion of patients, possibly because many patients were followedup in a primary care setting. $\mathrm{Hb}$ rose satisfactorily in most patients but many patients remained anaemic, especially in the underdosed subgroup, indicating a high iron need. Therefore, improvements in the areas of $\mathrm{Hb}$ follow-up and IV iron dosing could be beneficial. Overall, FDI was efficacious and well-tolerated.

63

EurOP $^{2}$ E - the European Open Platform for Prescribing Education, a consensus study among clinical pharmacology and therapeutics teachers

Mr. Michiel Janniek Bakkum ${ }^{1}$, Dr. Milan C. Richir ${ }^{1,2}$,

Prof. Paraskevi Papaioannidou ${ }^{2}$, Dr. Robert Likic ${ }^{2}$, Prof. Emilio J. Sanz ${ }^{2}$, 
prof. Thierry Christiaens ${ }^{2}$, Prof. João N. Costa ${ }^{2}$,

Prof. Romaldas Mačiulaitis Mačiulaitis ${ }^{2}$, Prof. Lorena Dima ${ }^{2}$,

Prof. Jamie Coleman ${ }^{2}$, Dr. Jelle Tichelaar ${ }^{1,2}$,

Prof. Michiel A. van Agtmael ${ }^{1,2}$

${ }^{1}$ Amsterdam University Medical Centers, VUmc, Amsterdam, The Netherlands

${ }^{2}$ European Association for Clinical Pharmacology and Therapeutics (EACPT) Education Working Group

Introduction: Sharing and developing digital educational resources and open educational resources has been proposed as a way to harmonize and improve clinical pharmacology and therapeutics (CPT) education in European medical schools. Previous research, however, has shown that there are barriers to the adoption and implementation of open educational resources.

Objectives: The aim of this study was to determine perceived opportunities and barriers to the use and creation of open educational resources among European CPT teachers, and possible solutions for these barriers. Methods: CPT teachers of British and EU medical schools completed an online survey. Opportunities and challenges were identified by thematic analyses and subsequently discussed in an international consensus meeting.

Results: Data from 99 CPT teachers from 95 medical schools were analysed. Thirty teachers $(30.3 \%)$ shared or collaboratively produced digital educational resources. All teachers foresaw opportunities in the more active use of open educational resources, including improving the quality of their teaching. The challenges reported were language barriers, local differences, lack of time, technological issues, difficulties with quality management, and copyright restrictions. Practical solutions for these challenges were discussed and include a peer review system, clear indexing, and use of copyright licenses that permit adaptation of resources.

Conclusion: Key challenges to making greater use of CPT open educational resources are a limited applicability of such resources due to language and local differences, and quality concerns. These challenges may be resolved by relatively simple measures, such as allowing adaptation and translation of resources and a peer-review system.

On the basis of these solutions a framework for the EurOP ${ }^{2} \mathrm{E}$ platform was developed (figure).

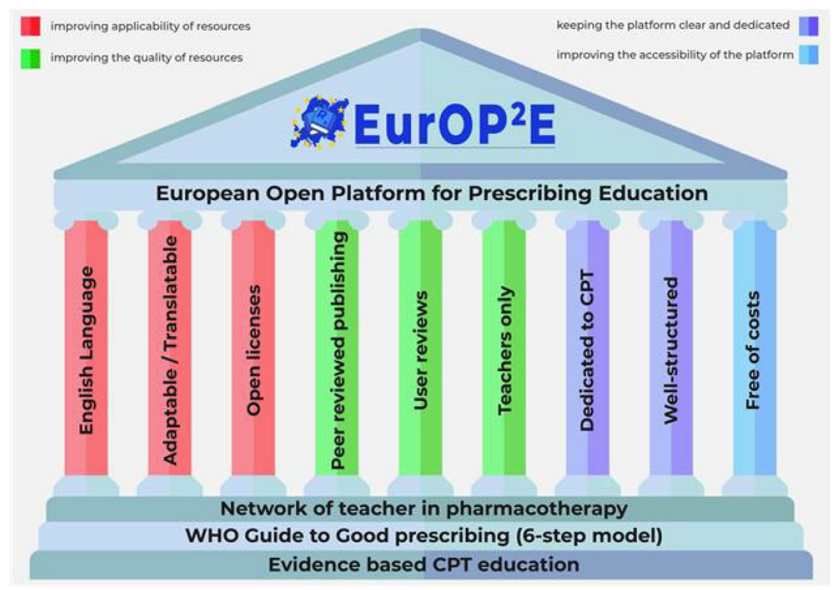

86

Factors influencing in-hospital prescribing errors: a systematic review

Rashudy F. Mahomedradja ${ }^{1}$, Michiel Schinkel ${ }^{2}$, Dr. Kim C.E. Sigaloff ${ }^{1}$, Michael O. Reumerman ${ }^{1}$, René Otten ${ }^{3}$, Dr. Jelle Tichelaar ${ }^{1}$, Prof. dr. Michiel A. van Agtmael ${ }^{1}$
${ }^{1}$ Department of Internal Medicine, Section Pharmacotherapy, Amsterdam UMC location VUmc, Amsterdam, the Netherlands

${ }^{2}$ Department of Internal Medicine, Section of Acute Medicine, Amsterdam UMC location VUmc, Amsterdam, the Netherlands

${ }^{3}$ Medical Information Specialist, University Library, Vrije Universiteit Amsterdam, Amsterdam, the Netherlands

Introduction: Several strategies reducing prescribing errors (PEs) and related patient harm have been developed over the past decades but with little succes. Insight and understanding of factors influencing this complex process is needed to effectively and sustainably reduce PEs and hereby drug related harm.

Objective: To review the literature to create an overview of all factors, both facilitating factors as well as protective barriers, influencing inhospital prescribing resulting in PEs.

Review methods and data sources: PubMed, EMBASE.com, and the Cochrane Library (via Wiley) were searched, based on the Preferred Reporting Items for Systematic Reviews and Meta-Analysis (PRISMA) statement, for studies that identified factors that influence in-hospital prescribing, from inception to 10 June 2020.

Results: 29 unique factors influencing in-hospital PEs were identified and categorized into 6 domains: prescriber, prescription, computerized systems, medication reconciliation, organization and communication. The domains 'prescriber-', 'organization-', 'computerized systems-' and 'prescription-related factors' are the top 4 . Overall, $82.8 \%$ of these unique factors identified, were found faciliting to in-hospital PEs. The most abundant facilitator was insufficient (drug) knowledge, poor prescribing skills, and/or inexperience of prescribers.

Conclusions: In-hospital PEs are influenced by multiple factors. To improve in-hospital prescribing, and reduce PEs, awareness at multiple domains is required: Prescriber, organization, communication, prescription, medication reconciliation, and computerized systems all play a role in the complex process of in-hospital prescribing. Protective barriers can become facilitators when not monitored regulary over time. Future strategies should have a multifaceted approach and include follow-up to assertain the desired effect.

\section{1}

Patients-Led Multi-Stakeholder National Educational Project on Rational Use of Medicaments in Slovakia - Medication with Sense Prof. MD, PhD, PhD. Jozef Glasa ${ }^{1}$, MD, PhD Helena Glasova ${ }^{1}$, PharmDr, MPH, PhD, MSc Dominik Tomek ${ }^{1}$, PhDr Maria Levyova ${ }^{2}$ ${ }^{1}$ Department of Clinical Pharmacology, Faculty of Medicine, Slovak Medical University in Bratislava, Bratislava, Slovak Republic

${ }^{2}$ Association on Protection of Patient Rights in Slovakia, Bratislava, Slovak Republic

Introduction: Empowerment of patients is ever more recognized as a key factor in achieving the goals of effective, sustainable medicinal drugs policies. A dedicated education of patients, their advocates, and of the general (lay) public is hereby deemed indispensable.

Objectives: Stemming from positive experiences of almost two decades of successful, multifaceted collaboration among the patients' organizations, academia, physicians' and pharmacists' organizations, enhanced by founding the EUPATI Slovakia platform; the nation-wide, patients-led, multi-stakeholder educational project, entitled „Medicaments with Sense", was launched in Slovakia (2017), to develop and strengthen previous successful initiatives.

Methods: The project activities focused upon (a) building or developing the necessary capacities, including (aa) accumulation of unbiased, layunderstandable medical information, and (ab) developing means of its effective updating and dissemination, and (b) providing the services for the actual users - patients, media, and public.

Results: The project was effective in: 1) networking and pooling the trusted quality information and educational resources available to the relevant stakeholders involved - medical societies (incl. Slovak Society 
of Clinical Pharmacology, its scientific guarantor), physicians'/pharmacists' associations, academia (incl. Institute of Pharmacology and Clinical Pharmacology, Slovak Medical University in Bratislava), and patients' organizations (Association on the Protection of Patients' Rights in Slovakia, its lead/coordinator), 2) ensuring support, incl. funding, of the regulator (Health Ministry), payers (health insurance companies), and (under strict ethical rules) pharma industry, 3) developing capacities for effective information dissemination (e. g. website, information network, ambassadors, media collaborations), 4) establishing dedicated activities for/with patients, incl. general public and media engagement (e. g. annual conference, prizes awarded to respected physicians and nurses, the network of "trustful doctors" and "trustful pharmacies" and others).

Conclusion: The results achieved by this ongoing patients-led initiative document the potential and usefulness of patients' empowerment in fostering the goals of rational medicinal drugs policies.

92

Physicians' assessment of the knowledge base prototype based on clinical guidelines: speed and correctness of treatment choice Prof Maxim Frolov ${ }^{1,2}$, Vladimir Rogov ${ }^{1,2}$

${ }^{7}$ Volgograd State Medical University, Volgograd, Russian Federation

${ }^{2}$ Volgograd Medical Scientific Center, Volgograd, Russian Federation

Introduction: Clinical decision support system (CDSS) are known to reduce errors and improve the quality of care. We, using the KBPublisher platform, have created a prototype of a knowledge base (KB) based on current clinical guidelines (CG) with the possibility of its subsequent use in CDSS. In the present study, we examined the opinion of doctors when comparing it with other methods of obtaining information. Objectives: to evaluate (in terms of speed, quality and convenience) the studied prototype of the knowledge base in comparison with the search on the website of the heading list of the Ministry of Health and the file service of CG in the health information system (HIS).

Methods: In a pilot randomized study among 30 general practitioners, the correctness of prescribing pharmacotherapy in solving a clinical case study was assessed. Then, group interviews were conducted in each group to obtain opinions and assessments of the digital service. The doctors were randomized to 3 equal groups: the first one was given access to the official resource http://cr.rosminzdrav.ru/, the second - to the KB files in the HIS file service, the third - to the service under study. The topics of the cases were limited to the information of 3 clinical guidelines.

Results: The minimum time and the maximum number of correct answers was shown by group 3. Almost the same time was shown by those doctors from group 1 who quickly found the required document. Group 2 doctors were slower to find the correct answer, 4 doctors did not complete the task on time. The conducted group interviews showed a high assessment of the interface and the search engine in group 3; interface but not search engine in group 1. Group 2 doctors noted only the high relevance of the information, but not the convenience and speed of the search.

Summary / Conclusions: A prototype knowledge base based on current clinical guidelines, created on the KBPublisher platform, allows you to quickly find information in the clinical decision support mode, has a convenient structure of higher detail than in CD document on the official website or in a separate file on the doctor's computer.

95

Influence of a Preferred Drugs Initiative on Prescribing Trends for Cardiovascular Medicines in Ireland

Ms Lorna Williams ${ }^{1}$, Ms Colette Morris ${ }^{2}$, Assoc Prof Helen Gallagher ${ }^{1}$ ${ }^{1}$ School of Medicine, Conway Institute, University College Dublin, Dublin, Ireland

${ }^{2}$ University Hospital Waterford, Waterford, Ireland
Introduction: The Preferred Drugs Initiative (PDI) was set up in Ireland via the Medicines Management Programme in 2013. It was designed to rationalise medicine use, with cost containment as a major aim. The Initiative recommends a single 'preferred drug' within a therapeutic class that prescribers are encouraged to choose as their first choice agent. Since its launch, ten preferred drugs in different classes have been selected and the initiative continues to expand. There are gaps in our knowledge of the impact of the PDI on prescribing trends in Ireland.

Objectives: This study aimed to examine the influence of the PDI on prescribing trends for five drug classes within the area of cardiovascular medicine: Statins, angiotensin-converting enzyme (ACE) inhibitors, angiotensin-II receptor blockers (ARBs), beta blockers and calcium channel blockers (CCBs). These drug classes are commonly used in the treatment and prevention of cardiovascular disease, a prevalent and costly cause of morbidity and mortality in Ireland and worldwide. The study aimed to analyse the use of all marketed drugs within these drug classes, determine the effect of the PDI on choice of agent, and consider why observed trends may have occurred.

Methods: An observational drug utilization study was performed by extracting data from the Primary Care Reimbursement Service, which runs the main public drug schemes in Ireland. This database records the number of dispensed prescriptions for each active pharmaceutical ingredient and it is a large and high-quality data source for measuring drug use in the adult Irish population. Most of the data was in the public domain and missing data were provided on request. The time period 2008-2020 was analysed, and this included several years before and after implementation of the PDI.

Results: Overall, there was little evidence to suggest that prescribers have adopted recommendations of the PDI. Notably, the preferred statin, simvastatin, was the least frequently prescribed drug both before and after its selection as the preferred agent. It has subsequently been replaced by atorvastatin, the most widely used statin. The preferred ACE inhibitor, ramipril, was the most commonly prescribed drug, but the PDI did not impact significantly on use of other ACE inhibitors. Although candesartan use increased slightly over this time period, as the preferred ARB it was placed fifth of six drugs in this class, with valsartan, losartan, olmesartan and telmisartan all being more popular. Bisoprolol was the most commonly dispensed beta blocker, before and after its selection as the preferred agent, and atenolol use notably decreased over the period of study. Although amlodipine was the most frequently used $\mathrm{CCB}$, its selection as the preferred CCB did not appear to abate the much increased use of lercanidipine in recent years.

Conclusion: Overall, there was little evidence that the PDI had been taken up by prescribers in the area of cardiovascular medicine. This study highlights the importance of reviewing national guidelines to ensure the quality of prescribing in Ireland. Clinical guidelines have the potential to improve decision making, reduce prescribing variation, contain costs and enhance pharmaceutical care.

99

Memantine misuse and diversion practices: a netnographic analysis of users' experiences published online

Doctor Salim Mezaache ${ }^{1}$, Doctor Perrine Roux ${ }^{1}$, Doctor Emilie Jouanjus ${ }^{2}$ ${ }^{1}$ SESSTIM, UMR INSERM-University of Aix Marseille; PACA Regional Health Observatory, Marseille, France

${ }^{2}$ Addictovigilance Center, Toulouse University Hospital; CERPOP, UMR INSERM-University of Toulouse, Toulouse, France

Introduction: Memantine, an NMDA receptor antagonist indicated in the treatment of Alzheimer's disease, presents pharmacodynamics similarities with commonly misused and diverted drugs including ketamine and dextromethorphan. However, there is, to our knowledge, no description of such practices with memantine. The objective was to characterize the consumption practices of memantine by implementing a netnographic study. 
Methods: We performed a netnographic study by applying ethnographic methods to publicly available online content of web forums. We identified the memantine users' experiences posted on English and Frenchwritten web forums. We anonymously extracted all related discussion threads, posted anytime until July 2019, except those reporting experiences in Alzheimer's disease. A qualitative thematic content analysis was performed.

Results: We included 128 discussion threads from 5 websites: reddit.com $(n=104)$; drugs-forum.com $(n=9)$; erowid.org $(n=8)$; bluelight.org $(n=5)$ and psychoactif.org $(\mathrm{n}=2)$. We classified the related content in six themes: i) Products, sourcing and decision making ( $\mathrm{n}=31)$; ii) Motivation for use ( $n=95)$; iii) Route of administration and doses ( $n=70)$; iv) Perceived effects and outcomes $(n=109)$; v) Comparison or combination to other drugs $(n=30)$; vi) Tolerance and abuse potential $(n=23)$. When details were available, users were mostly males, aged between 18 and 27 and experimented recreational drug users. They reported using memantine for therapeutic purposes or in search for recreational, dissociative effects; and described a limited abuse/addiction liability due to pharmacological features perceived as detrimental for misuse and to the easy access of more attractive substances.

Discussion: Our analysis highlighted two types of uses, either motivated by recreational or therapeutic purposes and characterized by broadly different practices. Although of lower magnitude than those observed with other dissociative drugs, memantine misuse and diversion practices exist and are shared online by users. The use of an innovative online ethnographic method is useful to describe previously unknown drug consumption practices from the users' perspective.

\section{Poster Session 4b - Toxicology/health technology/drug access}

\section{2}

Prescribing of oral anticoagulants for patients with atrial fibrillation of age $\geq \mathbf{6 5}$ years in Estonia - national quality indicator Dr Toomas Marandi ${ }^{1,2}$, Mrs Pille-Riin Vare ${ }^{3}$, Dr Tiia Ainla ${ }^{1,2}$,

Dr Katrin Martinson ${ }^{4}$, Mr Erki Laidmäe ${ }^{5}$, Associate professor Priit Kampus ${ }^{1,2}$

${ }^{1}$ North Estonia Medical Centre, Tallinn, Estonia

${ }^{2}$ Department of Cardiology, Institute of Clinical Medicine, University of Tartu, Tartu, Estonia

${ }^{3}$ Estonian Health Insurance Fund, Tartu, Estonia

${ }^{4}$ Linnamõisa Family Medicine Center, Tallinn, Estonia

${ }^{5}$ Estonian Health Insurance Fund, Tallinn, Estonia

Atrial fibrillation (AF) is the most common sustained cardiac arrhythmia in adults associated with substantial morbidity (t.e. increase of stroke, heart failure, impaired quality of life etc) and mortality. Routine use of oral anticoagulants (OACs) is recommended for patients with AF with CHA2DS2-VASc score values $\geq 2$ (men) and $\geq 3$ (women) for stroke prevention. According to data from different registries $70-80 \%$ of patients with age $>65$ years have high thromboembolic risk, thus recommendation to use OACs is almost universal. Proportion of AF patients with high thromboembolic risk who are prescribed oral anticoagulation is a national quality indicator in Estonia.

Objective: To study prescribing of OACs in daily doses for stroke prevention for patients with age $\geq 65$ years with AF (ICD-10 diagnosis I48).

Methods: List of patients of age $\geq 65$ years with documented diagnoses I48 (ICD-10th version) from year 2018 and alive on 01.01.2019 was obtained from Health Insurance Fund's database. From Estonian Medical Prescription Center data about prescribed daily doses for the period 01.01-31.12.2019 or until death was obtained. For lifeday coverage analysis daily doses of OACs for stroke prevention were used: warfarin ( $3 \mathrm{mg}$ or $5 \mathrm{mg}$, arbitrarily, OD) rivaroxaban (15 $\mathrm{mg}$ or $20 \mathrm{mg} \mathrm{OD}$ ), apixaban $2.5 \mathrm{mg}$ or $5 \mathrm{mg}$ $\mathrm{BD})$, dabigatran (110 $\mathrm{mg}$ or $150 \mathrm{mg} \mathrm{BD})$ and edoxaban (30 $\mathrm{mg}$ or $60 \mathrm{mg}$ OD). We excluded patients treated for other concomitant diseases by low molecular weight heparins (enoxaparin, dalteparin, nadroparin, bemiprarin); prescriptions with lower (inappropriate) doses of rivaroxaban and dabigatran if not used concomitantly with clopidogrel or ticagrelor were classified as missing-prescriptions. Results: There were 33951 patients (13466 men and 20485 women) with age $\geq 65$ years diagnosed AF during the year 2018 and alive in 01.01.2019. $26992(79.5 \%)$ of them had at least one prescription of OACs for year 2019. All cause mortality was $8.9 \%(n=3027)$ during followup.

Conclusion: Proportion of lifedays of patients with AF covered by prescription of oral anticoagulants in recommended doses for stroke prevention needs further improvement. In addition, dispensing of prescriptions at pharmacies needs further studies before implementing next targeted interventions on national level.

This work was supported by Estonian Research Council [PRG435]

\begin{tabular}{|llll|}
$\begin{array}{l}\text { Lifedays covered with } \\
\text { daily doses for stroke } \\
\text { prevention (\%) }\end{array}$ & Men (n, \%) & Women (n, \%) & Total (n, \%) \\
\hline$\geq 100$ & $4263,31.7$ & $6992,34.1$ & $11255,33.2$ \\
\hline $90-99$ & $2338,17.4$ & $3659,17.9$ & $5997,17.7$ \\
\hline $80-89$ & $1111,8.3$ & $1619,7.9$ & $2730,8.0$ \\
\hline $60-79$ & $1237,9.2$ & $1782,8.7$ & $3019,8.9$ \\
\hline $40-59$ & $890,6.6$ & $1134,5.5$ & $2024,6.0$ \\
\hline $20-39$ & $461,3.4$ & $628,3.1$ & $1089,3.2$ \\
\hline $1-19$ & $355,2.6$ & $523,2.6$ & $878,2.6$ \\
\hline 0 & $2811,20.9$ & $4148,20.2$ & $6959,20.5$ \\
\hline
\end{tabular}

31

Amoxapine alleviates gastrointestinal damages induced by mycophenolic acid in a mouse model

Ms Manon Jardou $^{1}$, Ms Djouher Firoud ${ }^{1}$, Dr Claire-Cécile Barrot ${ }^{1}$,

Ms Hélène Arnion ${ }^{1}$, Ms Emilie Pinault ${ }^{1}$, Mr François-Ludovic Sauvage ${ }^{1}$, Dr James Javellaud ${ }^{1}$, Pr Pierre Marquet ${ }^{1,2}$, Dr Roland Lawson ${ }^{\mathbf{1 , 3}}$

${ }^{1}$ Inserm, IPPRIT, CHU Limoges (U1248), Centre de Biologie et de Recherche en Santé (CBRS), Limoges, France

${ }^{2}$ Faculty of Medicine, University of Limoges, Limoges, France

${ }^{3}$ Faculty of Pharmacy, University of Limoges, Limoges, France

Introduction:Mycophenolic acid (MPA), which is one of the most widely used immunosuppressants, is often associated with gastrointestinal adverse effects. More than $30 \%$ of patients suffer from nausea, vomiting, abdominal pain, diarrhea, erosions or ulcerations. The underlying mechanisms are still poorly understood. Recent investigations have pointed to the possible contribution of intestinal dysbiosis. MPA treatment has been shown to favour the expansion of beta-glucuronidase $(\beta-G)$ expressing bacteria. This bacterial enzyme is involved in the intestinal backtransformation of mycophenolic acid glucuronide (MPAG), an inactive liver metabolite excreted in the bile, into MPA. Therefore, we suggest that local exposure to high concentrations of MPA could trigger gastrointestinal damages.

Objectives: This study investigated the effect of amoxapine, a wellknown antidepressant but also inhibitor of bacterial $\beta-\mathrm{G}$, in reducing intestinal exposure to MPA and preventing gastrointestinal damages induced by MPA in a mouse model.

Methods: Seven week old C57BL/6J (SOPF) mice were randomly assigned in 4 groups of 8 mice per group: (G1) vehicle, (G2) mycophenolate mofetil (MMF), a prodrug of MPA ( $900 \mathrm{mg} / \mathrm{kg} / \mathrm{day}),(\mathrm{G} 3)$ amoxapine $(5 \mathrm{mg} / \mathrm{kg} /$ day) and (G4) MMF + amoxapine. All the treatments were given 
once daily by oral gavage for 7 days. Fecal samples were collected on day 0 , day 4 and day 8 to access MPA and MPAG concentrations (LC-MS/MS). After the sacrifice (day 8), the proximal colon was harvested for histological analysis (hematoxylin-eosin-saffron staining).

Results: Concomitant treatment with amoxapine significantly decreased fecal MPA concentrations $(\mu \mathrm{g} / \mathrm{mL})[\mathrm{G} 2(5.99 \pm 3.69)$ vs G4 $(1.62 \pm 0.47) \mathrm{p}<$ 0.05 , Mann-Whitney test] with a slight decrease in MPAG concentrations [G2 $(1.23 \pm 0.50)$ vs G4 $(0.49 \pm 0.39) \mathrm{p}>0.05$, Mann-Whitney test] in mice. Structural changes induced by MMF in the proximal colon were also prevented by the combination with amoxapine [colon tissue/total section area $(\%)$ for G1 $(90 \pm 7)$ vs G2 $(68 \pm 8) \mathrm{p}<0.05 ; \mathrm{G} 1$ vs G3 $(85 \pm 6) \mathrm{p}>0.05$ and G3 vs G4 $(83 \pm 9)$ p $>0.05$; ANOVA, Bonferroni post-hoc].

Conclusion: Our results support the central role of local exposure to MPA in the pathophysiology of mycophenolate-induced enteropathy. Further investigations will decipher the cellular and molecular mechanisms.

43

Role of immune checkpoints and activated helper and cytotoxic

T-cells in drug-induced liver injury (DILI)

Alejandro Cueto-Sanchez ${ }^{1}$, Judith Sanabria-Cabrera ${ }^{1,2}$,

Mercedes Robles-Diaz ${ }^{1,3}$, Aida Ortega-Alonso ${ }^{1}$, Miren Garcia-Cortes ${ }^{1,3}$, Enrique del Campo-Herrera ${ }^{1}$, Rocio Gonzalez-Grande ${ }^{4}$,

Miguel Jimenez ${ }^{4}$, Francisco Ruiz-Cabello ${ }^{5}$, Raul J Andrade ${ }^{1,3}$,

Dr Camilla Stephens ${ }^{1,3}$, M Isabel Lucena $\mathbf{a}^{\mathbf{1}, 2,3}$

${ }^{1}$ Servicio de Farmacología Clínica and UGC A parato Digestivo, Instituto de Investigación Biomédica de Málaga-IBIMA, Hospital Universitario Virgen de la Victoria, Universidad de Málaga, Málaga, Spain

${ }^{2}$ Platform ISCIII for Clinical Research Clinical Trials UICEC-IBIMA, Málaga, Spain

${ }^{3}$ Centro de Investigación Biomédica en Red en el Área Temática de Enfermedades Hepáticas y Digestivas (CIBERehd), Madrid, Spain

${ }^{4}$ Servicio de Aparato Digestivo, IBIMA, Hospital Universitario Regional de Málaga, Málaga, Spain

${ }^{5}$ Servicio de Análisis Clínicos e Inmunología, UGC de Laboratorio Clínico, Hospital Universitario Virgen de las Nieves, Granada, Spain

Introduction: Idiosyncratic DILI is a challenging condition, believed to involve the immune system. This hypothesis is supported by the identification of various HLA risk alleles.

Objectives: To evaluate a potential role of the immune system in DILI through leukocyte immunophenotyping using flow cytometry.

Methods: Blood samples were collected from adjudicated DILI $(n=12)$ and viral hepatitis $(\mathrm{VH}, 13)$ at day 1 (recognition), day 7 and day $>30$. Blood samples from healthy liver controls (HLC, 54) were extracted at a single time point. Leukocyte populations and immune checkpoint expressions were determined based on cell surface receptors, except for CTLA-4 that was determined intracellularly, using multiparametric flow cytometry (BD FACSVerse).

Results: No differences were detected in leukocytes, lymphocytes or neutrophils cell counts at day 1 between the three populations. However, both DILI $(0.57 \times 10 \mathrm{E} 09 / \mathrm{L}, \mathrm{p}=0.037)$ and HV $(1.41 \mathrm{x} 10 \mathrm{E} 09 / \mathrm{L}, \mathrm{p}<0.0001)$ had increased monocyte levels compared with HLC $(0.35 \times 10 \mathrm{E} 09 / \mathrm{L})$. At day 1 DILI presented significantly higher levels of activated helper T-cells (CD4+/DR+) and activated cytotoxic T-cells (CD8+/DR+) than HLC ( $14 \%$ vs $6.3 \%, p<0.0001 ; 31 \%$ vs $15 \%, p=0.0003$, respectively). The same trend was detected for $\mathrm{VH}$. A strong correlation between activated CD4+ and CD8+ elevation was found in DILI $(r=0.85, p<0.001)$, but less in VH $(\mathrm{r}=0.58, \mathrm{p}=0.0015)$. With regards to helper T-cell subpopulations, DILI had significantly higher level of Th1 ( $52 \%$ vs $42 \%, \mathrm{p}=0.0358)$, while VH had lower level of Th9 compared with HLC ( $13 \%$ vs $18 \%, \mathrm{p}=0.0112)$. No other significant differences in lymphocyte populations were detected for DILI. Regarding immune checkpoint expressions on CD4+, DILI presented higher intracellular CTLA-4 level than HLC ( $28 \%$ vs $18 \%, \mathrm{p}=0.0192)$. $\mathrm{VH}$ presented higher level of both CTLA-4 $(30 \%$ vs $18 \%, \mathrm{p}=0.0068)$ and PD-1 (23\% vs $9.1 \%, p=0.0005)$ than HLC. Significantly higher expression of checkpoint ligand PD-1L on monocytes was also found in DILI (5.3\% vs $3.4 \%, \mathrm{p}=0.0452)$ and $\mathrm{VH}(9.1 \%$ vs $3.4 \%, \mathrm{p}<0.0001)$. The level of all leukocyte populations and checkpoint expressions in DILI and VH approached HLC levels in the later samples, except for CD28 and CD86 that are constitutively expressed.

Conclusion: Our findings suggest that an adaptive immune response is involved in DILI in which activated CD4+ and CD8+ T-cells play an important role. Increased expression of negative immune checkpoints and ligands reflects restoration of immune homeostasis. Funding: PI16/01748, PI19/00883, CIBERehd-ISCIII

48

PLASMA AND URINARY CONCENTRATIONS OF ARACHIDONIC ACID-DERIVED EICOSANOIDS ARE ASSOCIATED WITH DIABETIC KIDNEY DISEASE

Miss Sonia Mota Zamorano ${ }^{1}$, Mr Nicolás R Robles ${ }^{2}$,

Mr Juan López-Gómez ${ }^{3}$, Mrs Bárbara Cancho², Miss Luz M González', Mrs Guadalupe García-Pino ${ }^{4}$, Mrs María Luisa Navarro-Pérez,

Mr Guillermo Gervasini ${ }^{1}$

${ }^{1}$ Department of Medical and Surgical-Therapeutics, Division of Pharmacology, University of Extremadura, Badajoz, Spain

${ }^{2}$ Service of Nephrology, Badajoz University Hospital, Badajoz, Spain

${ }^{3}$ Service of Clinical Analyses, Badajoz University Hospital, Badajoz, Spain

${ }^{4}$ Service of Nephrology, Zafra Hospital, Zafra, Spain

${ }^{5}$ Department of Biomedical Sciences, University of Extremadura, Badajoz, Spain

Introduction: Diabetic kidney disease (DKD) is one of the main complications of diabetes mellitus and the most common cause of end-stage renal disease.

In the last years, some studies have pointed to the epoxygenase pathway of arachidonic acid (AA) metabolism as a route that might be deeply involved in the mechanisms underlying DKD.

Objective: We aimed to determine whether plasma and/or urinary levels of dihydroxyeicosatrienoic (DHETs) and 20-hydroxyeicosatetraenoic (20-HETE) acids are associated with DKD.

Methods: A total of 334 subjects (132 DKD patients and 202 nondiabetic individuals) were studied. Plasma levels of 11,12-DHET, 14,15-DHET and 20-HETE were measured by LC/MS/MS. Urinary 20-HETE concentrations were determined by immunoenzymatic assay.

Results: Subjects with normoalbuminuria had larger 20-HETE-tocreatinine urinary ratios $(20-\mathrm{HETE} / \mathrm{Cr})$ than those with micro and macroalbuminuria $(p=0.012)$. Likewise, participants with eGFR $>60 \mathrm{ml} /$ $\mathrm{min} / 1.732$ had higher plasma levels of 14,15 -DHET $(\mathrm{p}=0.039)$ and 20 HETE/Cr ratios $(\mathrm{p}=0.007)$. Concentrations of 14,15-DHET, 11,12-DHET and 20-HETE/Cr were significantly lower in DKD patients. Median values for non-diabetic vs. DKD were, respectively, 493 (351-691.5) vs. 358 (260.5-522) ng/L, p=3e-5; 262 (183.5-356) vs. 202 (141.5-278) ng/L, $\mathrm{p}=1 \mathrm{e}-4$ and $5.26(1.68-11.65)$ vs. $2.53(1.01-6.28) \mathrm{ng} / \mathrm{mgCr}, \mathrm{p}=0.010 . \mathrm{In}$ addition, 20-HETE/Cr ratios were higher in patients with non-proteinuric DKD than in those with typical DKD $(\mathrm{p}=0.020)$. When only individuals with impaired filtration were considered, 14,15-DHET and 11,12-DHET levels were still higher in non-diabetic subjects $(\mathrm{p}=0.002$ and $\mathrm{p}=0.006$, respectively).

Conclusions: Our results indicate that AA-derived eicosanoids may play a relevant role in DKD.

49

An experimental model to simulate binge ingestion of alcohol and energy drinks in a laboratory setting MD Olga Hladun ${ }^{1,2}, \mathrm{PhD}$, MD Clara Pérez-Mañá ${ }^{1,2}$, PhD, MD Esther Papaseit ${ }^{1,2}$, PhD student Lourdes Poyatos ${ }^{1,2}$, 
RN Soraya Martin ${ }^{1}, \mathrm{PhD}, \mathrm{MD}$ Ana Maria Barriocanal ${ }^{1,2}$, $\mathrm{PhD}$, MD Magí Farré ${ }^{1,2}$

${ }^{1}$ Department of Clinical Pharmacology, Hospital Universitari Germans Trias i Pujol and Institut de Recerca Germans Trias i Pujol (IGTP), Badalona, Spain

${ }^{2}$ Department of Pharmacology, Therapeutics and Toxicology, Universitat Autònoma de Barcelona (UAB), Cerdanyola del Vallès, Spain

Introduction: Binge drinking consists in drinking alcoholic beverages with an intention of becoming intoxicated by heavy consumption of alcohol, reaching blood alcohol concentrations of $\geq 80 \mathrm{mg} / \mathrm{dl}$ in a short period of time. It usually corresponds to $\geq 4$ drinks in women and $\geq 5$ drinks in men in $2 \mathrm{~h}$. Binge-drinking is becoming more popular in young worldwide. Energy drinks (ED) are often mixed with alcohol (AmED) with the intention of counteracting alcohol effects with the caffeine they contain. AmED consumption is associated with elevated rates of binge drinking and impaired driving when compared with alcohol alone. The acute effects of this mix using this pattern of consumption should be investigated in both genders.

Objectives: The aim of the study was to evaluate an experimental model to assess the acute effects and alcohol concentrations after controlled administration of AmED with a binge-drinking pattern.

Methods: A double-blind, placebo-controlled crossover pilot study was conducted in 6 healthy volunteers with 3 different treatment conditions: alcohol + ED, alcohol + placebo of ED, and both placebos. Alcohol doses were $65 \mathrm{~g}$ in men and $50 \mathrm{~g}$ in women and were mixed with $750 \mathrm{ml}$ of ED in men and $575 \mathrm{ml}$ in women. Total volume was divided in 6 equal doses each one administered every 15 minutes. Alcohol concentrations in breath air were measured along 10 hours. Additionally subjective effects (Visual Analogue Scales, Biphasic Alcohol Effects Scale (BAES), physiological effects and driving-related skills were assessed.

Results: Participants (3 men:3 women) had an average age and BMI of 23.8 years and $22.2 \mathrm{~kg} / \mathrm{m} 2$, respectively. Alcohol peak concentration in exhaled air was $0.39 \pm 0.06 \mathrm{mg} / \mathrm{l}$, corresponding to nearly $80 \mathrm{mg} / \mathrm{dl}$ in blood. No gender differences were observed despite the lower dose in women. The drunkenness feelings were mild $(37.3 \pm 19.6 \mathrm{~mm}$ in men and $32.5 \pm 25.0$ in women) and were detected up to 6 hours later. Greater drowsiness was observed with alcohol alone in comparison with alcohol+ED. Both conditions with alcohol produced mild activation and sedation in BAES. Regarding mean reaction time at $1.75 \mathrm{~h}$ a more pronounced increase was observed with alcohol alone, and in women (75.1 $\pm 52.8 \mathrm{~ms}$ ). Alcohol \pm ED reduced diastolic blood pressure and increased heart rate the first 3 hours. No serious adverse effects were observed.

Conclusions: Breath alcohol concentrations did not clearly reach those defining binge drinking in some participants. Women had similar peak alcohol concentrations and subjective effects than men. We decided to increase alcohol dose in our definitive study in order to simulate more precisely an experimental binge drinking pattern. Preliminary results confirm that the objective has been achieved increasing the dose to $70 \mathrm{~g}$ and $55 \mathrm{~g}$ in men and women, respectively.

Acknowledgements: Ministerio de Sanidad, Política Social e Igualdad, Plan Nacional Sobre Drogas (PNSD 2018 I037 and PNSD 2016I024), Instituto de Salud Carlos III (Red de Trastornos Adictivos ISCIIIFEDER RD16/0017/0003 and Predoctoral grants FI18/00179 Lourdes Poyatos) and AGAUR Gencat Suport Grups Recerca (2017 SGR 316).

57

Reimbursement of drugs for ultra-rare diseases in Lithuania Egle Karinauske ${ }^{1}$, Tomas Lasys ${ }^{1}$, Skaiste Kasciuskeviciute ${ }^{1,2}$, Akvile Usaite $^{1}$, Simona Stankeviciute ${ }^{1,2}$, Vladas Sadauskas ${ }^{1}$

${ }^{1}$ State Medicines Control Agency of Lithuania, Vilnius, Lithuania

${ }^{2}$ Lithuanian University of Health Sciences, Kaunas, Lithuania

Introduction and objectives: Drugs for ultra-rare diseases in Lithuania are reimbursed on a named-patient basis. The hospital in which patient is being treated can request to receive a non-reimbursed or off-label treatment if the disease's incidence is 1 per 200,000 people in Lithuania. Other criteria for reimbursement include the drug having orphan drug designation by European Medicines Agency (EMA), authorized indication in European, Lithuanian or any other European country's national registry and clinical evidence. All this information is presented by the hospital and is evaluated by the State Medicines Control Agency Health Technology Assessment (HTA) unit, and then the deliberation is made at the Committee for reimbursement of ultra-rare diseases (Committee). The Committee makes the final decision if a drug should be reimbursed. Our main objective of this study was to analyse submission evaluation and decision-making processes.

Methods: This cross-sectional survey was conducted during the sixmonth period (August 1st, 2020 till January 31st, 2021). HTA unit was responsible for evaluation of the drug according to the criteria (see above) and assessment of the clinical data (shown as direct endpoints). HTA unit's evaluation does not involve cost and budget impact assessment. Additionally, we checked what the decision of the Committee was. The results are presented as descriptive statistics.

Results: During the six-month period, HTA unit evaluated 33 drugs for ultra-rare diseases. 12 out of the 33 of the requested drugs were intended for haematological or oncological diseases. 7 out of the 33 drugs had orphan designation by the EMA. 19 out of the 33 drugs had authorized indication in European, Lithuanian or any other European country's national registry, other drugs sought to be reimbursed off-label. 21 out of the 33 drugs were evaluated in clinical trials, clinical cases or guidelines using direct clinically relevant endpoints, such as overall survival (for haematological or oncological diseases), improvement of symptoms or the quality of life. HTA unit recommended to reimburse 26 out of the 33 of drugs. The Committee decided to reimburse 15 out of the 33 drugs, not to reimburse 11 out of the 33 and for the remaining seven, the decision is not yet made at the time of this abstract submission. Out of 15 positive Committee's decisions, HTA unit recommended to reimburse 12. Out of 11 negative Committee decisions, the HTA unit recommended to reimburse 7 (Figure 1).

Conclusions: Lithuanian definition of ultra-rare diseases ( 1 out of 200,000 people) does not correlate with European Commission's definition of orphan drugs (1 out of 2000 people). Only one-fifth of evaluated drugs had orphan designation by the EMA. One-third of the drugs sought to be reimbursed for haematological or oncological diseases. More than a half of drugs had authorized indications which, in our opinion, correlates with clinical evidence (direct endpoints). There was a little correlation between HTA unit's recommendations and Committee's decisions. This shows that Committee considers more than clinical evidence, including societal perspective, cost, and budget impact.

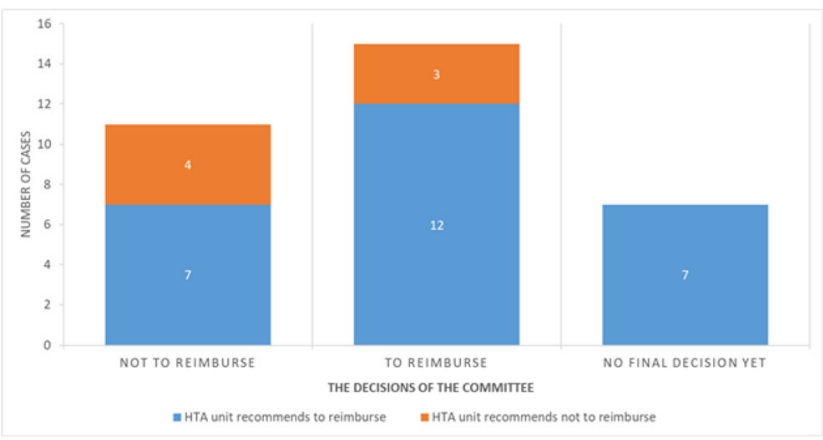

60

Accessibility and effectiveness of Spanish special access programs to pertuzumab (P) / trastuzumab emtansine (T-DM1) Almudena Ramirez-Garcia ${ }^{1}$, Concepción Payares-Herrera ${ }^{1}$, Elena Diago-Sempere ${ }^{1}$, Antonio Caballero-Bermejo ${ }^{1}$, 
Arantxa Sancho-Lopez ${ }^{1}$, Laura Javaloyes-Garachana ${ }^{1}$,

Gustavo Centeno Soto $^{1}$, Belén Ruiz-Antoran ${ }^{1}$, Cristina Avendaño-Solá ${ }^{1}$

${ }^{1}$ Universitary Hospital Puerta De Hierro Majadahonda, Spain

Introduction: Before medicinal products are marketed in the EU, patients with unmet medical needs can benefit from them under early access (EA) or compassionate use (CU) programs. These real-world patients may differ from those included in clinical trials. Therefore, EA/CU programs pose an opportunity to assess the real-world effectiveness.

Objectives: To evaluate the effectiveness, safety and accessibility of TDM1 and P in Spanish patients with metastatic HER2+ breast cancer $(\mathrm{MBC})$ treated under $\mathrm{EA} / \mathrm{CU}$ programs.

Methods: Retrospective, post-authorization, observational study, conducted from July 2018-December 2019. All Medical Oncology departments were invited to participate. Patients with HER2+ MBC receiving these medicinal products in EA/CU programs were eligible. Data were obtained from individual medical records and/or pre-existing special access registries and entered in an anonymized database. Approval was granted by Research Ethics Committee at Hospital Puerta de Hierro.

Results: 36 Medical Oncology departments participated and provided data for all their patients; 210 patients were included and analysed (40 from CU, 170 from EA programs), 29.6\% of the overall 709 patients treated with $\mathrm{P}$ or T-DM1 under EA/CU in Spain

Effectiveness: $42(20 \%)$ complete, $55(26.2 \%)$ partial responses and 40 (19\%) stable disease cases were observed. Median progression-free survival (independently assessed) was 14.1 (T-DM1) and 24.1 (P) months. Clinical benefit (complete/partial response or stable disease for $\geq 6$ months) was observed in $8.9 \%$ (T-DM1) and $13.3 \%(\mathrm{P})$.

Safety: No new/concerning findings were seen.

Accessibility: Mean time from physician's treatment decision to administration was 26.4 days (1-162). Most time was spent on local administrative procedures either prior to submitting the request to the Spanish Medicines Agency (54\%) or between approval receipt and treatment administration (33.9\%). No significant differences were noted per region or type of program.

Conclusion: Effectiveness was lower than reported in the literature, probably due to patients' clinical situation, but considered clinically relevant. Despite the general agreement on the need to speed up access to EA/CU, local intervention unrelated to the physician and patient decision appears as an important hurdle and accounts for a delay that could affect patient's health and mental wellbeing.

67

The use of surrogate endpoints in health technology assessment for oncology drugs

Tomas Lasys ${ }^{1}$, Egle Karinauske ${ }^{1}$, Skaiste Kasciuskeviciute ${ }^{1,2}$,

Akvile Usaite $^{1}$, Simona Stankeviciute ${ }^{1,2}$, Vladas Sadauskas ${ }^{1}$

${ }^{T}$ State Medicines Control Agency of Lithuania, Vilnius, Lithuania,

${ }^{2}$ Lithuanian University of Health Sciences, Kaunas, Lithuania

Introduction and objectives: Health technology assessment (HTA) in Lithuania began in July 2019 with a purpose to provide robust information for decision-makers about drugs which sought to be reimbursed. During the last years, an increase of submissions of medicines for oncological diseases was observed. The rise of treatment options in the field of oncology and prolonged overall survival (OS) for the patients, make the collection of the OS data too slow for demand. Progression-free survival (PFS) is often chosen as primary endpoint in clinical trials for faster availability of evidence of effectiveness of these drugs. Thus, most of the received submissions dossiers were built upon data of surrogate endpoints which does not show direct value for the patient's health. The main objective of this study was to analyse HTA submissions for oncological diseases and the endpoints used in clinical trials.

Methods: This cross-sectional survey was conducted during the 18month period (from July 17th, 2019 to December 31st, 2020). State
Medicines Control Agency of Lithuania HTA unit was responsible for evaluation of the dossiers of medicines applying for reimbursement. Out of all the submissions, HTA unit elected those where medicines were indicated for oncological disease. Then we analysed these submissions, if the direct or surrogate endpoint were reached in the randomised clinical trial (RCT) or network meta-analysis. Direct endpoints which were suitable were OS and QoL. The results are presented as descriptive statistics. Results: During the remainder of 2019 and 2020 HTA unit received a total of 33 submissions. Most of the submissions were for oncology products $(50 \%(4 / 8)$ and $60 \%$ (15/25) for each year, respectively). Out of the 19 submissions for oncology treatments, 68.4\% (13/19) of dossiers were based on RCTs where the primary endpoint of the study was a surrogate endpoint, $21.1 \%$ (4/19) of submissions were based on RCTs that had coprimary endpoints where one of them was OS, and only 2/19 of dossiers were based on RCT where primary endpoint was OS. Out of those studies, only $6 / 19$ showed mature statistically significant prolongation of OS and $1 / 6$ of them showed statistically significant QoL differences with comparator when measured with QoL questionnaire (EORTC QLQ). Only one of additional study, that had no mature OS data (1/13), also showed statistically significant QoL when measured with EORTC QLQ. However, no significant difference in QoL was detected in any of the assessed medicines when QoL was measured with another QoL questionnaire (EQ-5D) which is preferable in Lithuania.

Conclusions: One-third of all submissions provided statistically significant data of OS prolongation and only one-tenth provided proof of better QoL than comparator. However, in both cases statistically significant increment in QoL which was detected using EORTC QLQ questionnaire was not detected using EQ-5D QoL questionnaire which is preferable in Lithuania. Thus, none of the medicines showed better quality of life. So, our study showed that there is a lack of conventional evidence that shows direct value for the patient's health in the field of oncology.

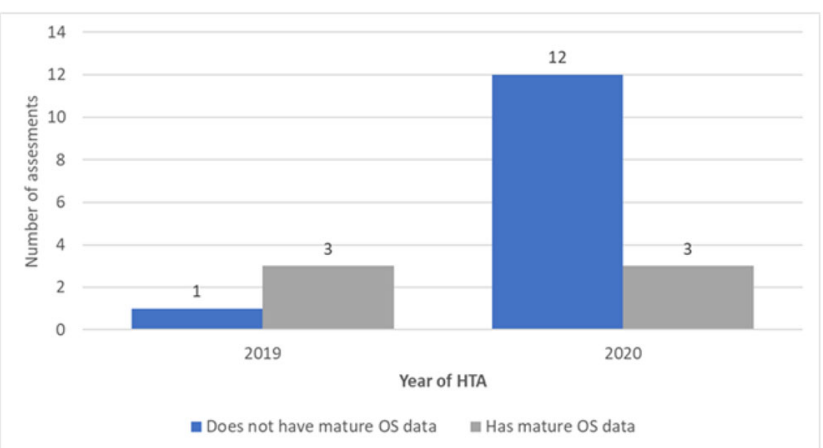

\section{S5- Precision medicine}

70

Resistant Hypertension improved by measuring drug levels to identify non-adherence: an interim-analysis

MSc Laura E.J. Peeters ${ }^{1}$, dr Mariette Kappers ${ }^{2}$, dr Jeroen van der $\mathrm{Net}^{3}$, dr Simone Hartong ${ }^{3}$, BSc Soma Bahmany ${ }^{1}$, dr Emma Massey ${ }^{1}$, prof Liset van Dijk ${ }^{4}$, prof Teun van Gelder ${ }^{1}$, dr Birgit Koch ${ }^{1}$, dr Jorie Versmissen ${ }^{1}$

${ }^{1}$ Erasmus Medical Center, Rotterdam, the Netherlands

${ }^{2}$ Amphia Hospital, the Netherlands

${ }^{3}$ Albert Schweitzer Hospital, Dordrecht, the Netherlands

${ }^{4}$ Netherlands Institute for Health Services Research, the Netherlands

Introduction: Identification of non-adherence to antihypertensive drugs is crucial to improve resistant hypertension (RH). We previously developed a reliable and convenient method to measure drug levels in blood by 
means of a dried blood spot (DBS) to identify non-adherence to antihypertensive drugs objectively.

Objective: The primary objective of this analysis is to determine whether drug levels measured with a DBS method combined with personalized feedback leads to a decrease in blood pressure in patients with RH and therefore decrease in prevalence of $\mathrm{RH}$ after 3 months due to an increase in adherence.

Method: This is a multi-center single-blinded randomized controlled trial (RHYME-RCT, NL6736) performed in 11 hospitals in the Netherlands. Patients went to an eligibility visit, where DBS sampling and a 24-hour ambulatory blood pressure measurement (ABPM) was performed simultaneously. Patients with a daytime systolic blood pressure (SBP) $>135$ and/or diastolic blood pressure (DBP) $>85 \mathrm{mmHg}$ were randomized to standard treatment (control) or intervention. The intervention was performed by the treating physician and included information on drug levels and a personalized feedback conversation based on a feedback tool. The follow-up period was one year and included visits at 3,6 and 12 months after the eligibility visit. At each visit an ABPM and DBS were performed.

Results: A total of 53 patients (mean age of $59 \pm 11$ years, $78.7 \%$ male) with at least three months follow-up time were included. During the follow-up blood pressure decreased in the intervention arm (SBP 4.0 $\mathrm{mmHg}$, DBP $3.3 \mathrm{mmHg}, \mathrm{n}=24$ ) as well as in the control arm (SBP 7.4 $\mathrm{mmHg}$, DBP $6.2 \mathrm{mmHg}, \mathrm{n}=29$ ). The prevalence of $\mathrm{RH}$ decreased from $100 \%$ in both arms to $75.0 \%$ in the intervention arm and $58.6 \%$ in the control arm. No improvements were seen in adherence rates over time. Furthermore, no significant differences were found after three months between the two groups in the degree of $\mathrm{RH}(\mathrm{p}=0.214)$, SBP $(\mathrm{p}=0.551)$ or adherence $(\mathrm{p}=0.746)$. However, the prevalence of RH over time improved in both the intervention ( $\mathrm{p}=0.014)$ and control arm $(\mathrm{p}=0.001)$.

Conclusion: Measuring blood pressure and drug levels led to improvement of blood pressure and thereby decrease in the prevalence of $\mathrm{RH}$. However, this improvement could not be linked to the actual intervention or improvement of adherence.

\section{5}

A model-based approach to describe creatinine kinetics in critically ill patients and its application in population pharmacokinetics

Mr. Sami Ullah ${ }^{1,2}$, Dr. Michael Zoller ${ }^{3}$, Prof. Ulrich Jaehde ${ }^{2}$, Mr. Mikayil Huseyn-Zada ${ }^{3}$, Dr. Thomas Weig ${ }^{3}$, Prof. Uwe Fuhr ${ }^{1}$, Dr. Usman Arshad ${ }^{1,2}$, Dr. Johannes Zander ${ }^{4}$, Dr. Max Taubert ${ }^{1}$

${ }^{1}$ University of Cologne, Faculty of Medicine and University Hospital Cologne, Center for Pharmacology, Department I of Pharmacology, Cologne, Germany

${ }^{2}$ Institute of Pharmacy, Clinical Pharmacy, University of Bonn, Bonn, Germany

${ }^{3}$ Department of Anaesthesiology, Hospital of the Ludwig Maximilians University (LMU) of Munich, Munich, Germany

${ }^{4}$ Institute of Laboratory Medicine, Hospital of the Ludwig Maximilians University of Munich, Munich, Germany

Introduction: Estimation of renal function by creatinine clearance $(\mathrm{CrCL})$ in critically ill patients is not trivial because renal function often change rapidly. Standard equations such as Cockcroft-Gault cannot be used as they assume steady state conditions. The current gold standard in such patients is measured $\mathrm{CrCL}$ ( $\mathrm{mCrCL}$ ), but it does not take urine collection errors into account. In contrast, nonlinear mixed effects (NLME) models can be used to describe dynamic changes in renal function and may also incorporate measurement errors.

Objectives: To develop a NLME model of creatinine which can be applied in non-steady state conditions; to model time-dependent changes in respective creatinine parameters; and to compare estimates by the creatinine model and by standard equations as a covariate in population pharmacokinetic models.
Methods: A compartmental creatinine model in critically ill patients was developed using NONMEM and predictors of CrCL were evaluated via step-wise covariate modeling. The ability of the model to describe changes in $\mathrm{CrCL}$ was assessed by simulations. Finally, model-based estimates of $\mathrm{CrCL}$ were used as a renal function index in a Population PK analysis of two renally excreted antibiotics.

Results: Based on data from 138 critically ill patients (530 serum and 373 urine creatinine observations), a one-compartment model with zero-order creatinine generation rate (CGR) and first-order CrCL was developed. As compared to the classical approach of handling inter-occasion variability, an autoregressive approach improved the model ( $\triangle$ AIC -29.9). Weight and sex were significantly related to CGR, while age, fat-free mass, plasma urea concentration, and liver transplant were linked to $\mathrm{CrCL}$. The model-based CrCL estimates were significantly superior to typical approaches including Cockcroft-Gault, $\mathrm{mCrCL}$, 4-variable modification of diet in renal disease (MDRD), 6-variable MDRD, chronic kidney disease epidemiology collaboration (CKD-EPI) and Berlin initiative study 1 (BIS1) as a renal function index to describe cefepime and meropenem PK (objective function values [OFV] 5761.1, 5811.1, 5842.6, 5819.3, 5809.2, 5817.7 and 5821.4, respectively). An R shiny application was developed for estimation of CrCL in critically ill patients (https:// taubertm.shinyapps.io/ICU_CRCL/).

Conclusion: The model-based approach can describe the unstable $\mathrm{CrCL}$ in critically ill patients better than the standard equations, and can be easily incorporated into Population PK evaluations.

\section{Late Breaking Abstracts}

103

Drug-drug interactions in patients admitted to the hospital via the emergency department: Preliminary results of a cross-sectional study

PharmDr. Zuzana Očovská ${ }^{1}$, PharmDr. Martina Maříková ${ }^{1,2}$, Kateřina Kukrálová1 $^{1}$, prof. Jiř́i Vlček ${ }^{1,2}$

${ }^{1}$ Department of Social and Clinical Pharmacy, Faculty of Pharmacy in Hradec Králové, Charles University, Hradec Králové, Czech Republic

${ }^{2}$ Department of Clinical Pharmacy, Hospital Pharmacy, University Hospital Hradec Králové, Hradec Králové, Czech Republic

Introduction: The presence of potential drug-drug interactions (DDIs) is common in daily practice and only a small proportion of potential DDIs result in hospitalization of patients.

Objectives: This study aims to identify DDIs in the medication history of patients acutely admitted to University Hospital Hradec Králové (Czech Republic) via the emergency department in August-November 2018. Primary outcomes include the prevalence of hospital admissions with at least one potential DDI in medication history and the prevalence of hospital admissions with at least one manifest DDI that contributed to the hospital admission.

Methods: The design of this study was cross-sectional. The following data were obtained retrospectively from electronic medical records: demographic data, medication history, medical history, laboratory and clinical findings and information about hospital admission. The identification of potential DDI was performed using Micromedex, Lexicomp (via UpToDate) and DrugAgency a.s. database of DDIs. A potential DDI was defined as a DDI with at least moderate severity category in at least one drug interaction database. The causality assessment was performed using Drug Interaction Probability Scale (DIPS). A manifest DDI that contributed to the hospital admission was defined as a DDI with clinical manifestation related to the main or contributory reason of hospital admission with a DIPS score of at least 2 points.

Results: A sample of 375 hospital admissions has been analyzed so far. Based on the preliminary findings, 2273 potential DDIs were identified in 258 hospital admissions. The prevalence of hospital admissions with at least one potential DDI in medication history was $68.8 \%$ (95\% CI: 64.1-73.5). 866 different DDI pairs were 
involved in these potential DDIs. Manifest DDIs which contributed to hospital admissions were identified in 17 (4.5\%) hospital admissions.

Conclusion: These preliminary results indicate that potential DDIs far outnumber manifest DDIs. However, given how significant the outcome is, the research on DDI-related hospital admissions deserves more attention.

The project is supported by Charles University (project SVV 260 551, project GA UK 14120).

\section{4}

Atezolizumab and severe cutaneous adverse reactions: a disproportionality analysis using FDA Adverse Event Reporting System (FAERS)

Dr. Alessandro Pecere ${ }^{1}$, Dr. Giulia Carlotta Bisinella ${ }^{2}$

${ }^{1}$ Department of Pharmaceutical Science - Università Degli Studi Di Milano, Milano, Italy,

${ }^{2}$ Department of Physiology and Pharmacology - Università degli Studi di Roma "La Sapienza", Roma, Italy

Introduction: Atezolizumab is a humanized IgG1 monoclonal antibody that targets PD-L1, used in the treatment of different cancer types. Recently, a warning has been issued by the European Medicine Agency regarding the risk of Steven-Johnson syndrome (SJS) and toxic epidermal necrolysis (TEN) in patients treated with atezolizumab. SJS and TEN, along with acute generalized exanthematous pustulosis (AGEP) and drug reaction with eosinophilia and systemic symptoms (DRESS), are classified as severe cutaneous adverse reactions to drugs (SCARs).

Objectives: The objective of this study was to investigate whether a disproportionally elevated signal of developing SCARs may be detected in patients treated with atezolizumab as compared to those treated with other drugs.

Methods: A retrospective analysis of spontaneously reported cases of SCARs contained in the free and publicly available database FDA Adverse Event Reporting System (FAERS) was conducted in the period 2014-2020. As a signal of disproportionality, we calculated the reporting odds ratio (ROR) and the relative $95 \%$ confidence interval $(\mathrm{CI})$ of SCARs in patients treated with atezolizumab. The signal was considered significant when the ROR lower limit of the $95 \%$ CI was $>1$

Results: A total of 13,011,377 FAERS reports were identified during the study period; $9,574(0.07 \%)$ reports mentioned atezolizumab. There were $26,572(0,20 \%)$ reports of SCARS of which 36 $(0.14 \%)$ attributed to atezolizumab treatment: 25 SJS, 3 DRESS, 7 TEN and 1 AGEP, respectively. The majority of these reports referred to patients aged $68-85$ years $(47.06 \%)$ and female $(58.82 \%)$. A significant signal was only detected for SJS: ROR $=4.74 ; 95 \% \mathrm{CI}$ 3.20-7.03

Conclusions: With this study we demonstrated that in patients treated with atezolizumab, SJS was the only SCAR for which a signal of disproportionate reporting was found. Although rare, SCARs are potentially fatal events and are now considered to be an identified risk for atezolizumab. It would be of interest to further analyze reports of SCARs associated with this drug, comparing signals deriving from FAERS and those deriving from the European pharmacovigilance database EudraVigilance.

106

Was it necessary to change therapeutic range of topiramate?

Ph.D. Blanka Koristkova ${ }^{1,2}$, Prof. M.D. Ph.D. Milan Grundmann ${ }^{1,2}$, Ph.D. Hana Brozmanova ${ }^{1,2}$, AssocProf, MD., Ph.D. Ivana Kacirova ${ }^{1,2}$

${ }^{1}$ Dept. Clin. Pharmacol., Faculty of Medicine, University of Ostrava, Ostrava, Czech Republic
${ }^{2}$ Div. Clin.1 Pharmacol, Dept. of Lab. Medicine, University Hospital Ostrava, Ostrava, Czech Republic

Introduction: The Norwegian Association for Clinical Pharmacology in their National Guidelines decreased reference range of topiramate from $5-20 \mathrm{mg} / \mathrm{L}$ to $2-10 \mathrm{mg} / \mathrm{L}$.

Objective: To ascertain which range produces better clinical outcomes for patients.

Methods: Data source were request forms for routine therapeutic drug monitoring of topiramate. Concentration dependent adverse drug reactions (ADR) were evaluated in 1,721 samples taken predose from 629 patients ( $39 \%$ children $<15$ years). Seizure frequency was recorded in 1,096 samples. Analysis was performed in 294 samples of monotherapy. Statistics: Prism 5.0, GraphPad Instatt: Mann-Whitney U test for median plasma level (PL). $\chi 2$-test for seizure frequency and for distribution of PL according to therapeutic range $5-20 \mathrm{mg} / \mathrm{L}$ and intervals $<2,2-5,5-10,10-20,>20 \mathrm{mg} / \mathrm{L}$. Fisher's exact test for ADR.

Results: Better seizure control was found in children both in whole cohort, as well as in monotherapy; in children with PL within $5-20 \mathrm{mg} / \mathrm{L}$ compared to $<5 \mathrm{mg} / \mathrm{L}$ and them with PL within $5-10 \mathrm{mg} / \mathrm{L}$ vs $<2 \mathrm{mg} / \mathrm{L}$ (Table). Seizure-free children had higher PL than those with seizure yearly: median (lower, upper quartile) [mg/L]: 5.5 (3.4-6.5) vs 4.7 (4.3-7.95). No difference was found in adults. Seizure control was poorer in all patients with $\mathrm{PL}<2 \mathrm{mg} / \mathrm{L}$ compared to $5-10 \mathrm{mg} / \mathrm{L}$; and $10-20 \mathrm{mg} / \mathrm{L}$; further in PL within 5-10 mg/L vs $10-20 \mathrm{mg} / \mathrm{L}$. Seizure control was also poorer in the period 2003-2006. ADRs reported in 38 samples $(2.8 \%)$ were without relation to PL.

Conclusion: Seizure control was significantly lower in PL $<2 \mathrm{mg} / \mathrm{L}$, in PL within $2-5 \mathrm{mg} / \mathrm{L}$ was not improved, while significantly better in PL within $10-20 \mathrm{mg} / \mathrm{L}$ compared to $5-10 \mathrm{mg} / \mathrm{L}$ with no difference in ADR. Therefore, change of therapeutic range from $5-20 \mathrm{mg} / \mathrm{L}$ to $2-10 \mathrm{mg} / \mathrm{L}$ is not recommended.

Table Seizure frequency.

* compared to 2003-2005, $\dagger$ compared to $<2 \mathrm{mg} / \mathrm{L}$, $\$$ compared to $5-10$ $\mathrm{mg} / \mathrm{L}$ all patiens (children+adult)

\begin{tabular}{|c|c|c|c|c|c|}
\hline & daily & monthly & yearly & without & $P$ \\
\hline \multicolumn{6}{|l|}{ all samples } \\
\hline children & $82(16 \%)$ & $89(18 \%)$ & $82(16 \%)$ & $244(49 \%)$ & $<0.0001$ \\
\hline adult & $55(9 \%)$ & $181(31 \%)$ & $137(23 \%)$ & $218(37 \%)$ & \\
\hline \multicolumn{6}{|c|}{ monotherapy } \\
\hline children & $11(8 \%)$ & $20(14 \%)$ & $30(22 \%)$ & $77(56 \%)$ & $<0.0001$ \\
\hline adult & $7(4 \%)$ & $29(19 \%)$ & $52(33 \%)$ & $68(44 \%)$ & \\
\hline \multicolumn{6}{|l|}{ period/all } \\
\hline 2003-2005 & $29(18 \%)$ & $59(36 \%)$ & $35(22 \%)$ & $39(24 \%)$ & \\
\hline 2006-2011 & $106(12 \%)$ & $201(23 \%)$ & $171(19 \%)$ & $408(46 \%)$ & $<0.0001^{*}$ \\
\hline 2012-2018 & $3(7 \%)$ & $10(24 \%)$ & $13(31 \%)$ & $16(38 \%)$ & NS \\
\hline \multicolumn{6}{|c|}{ period/monotherapy } \\
\hline 2003-2005 & $5(19 \%)$ & $5(19 \%)$ & $5(19 \%)$ & $1(44 \%)$ & \\
\hline 2006-2011 & $11(5 \%)$ & $35(15 \%)$ & $64(28 \%)$ & $120(52 \%)$ & $<0.0001^{*}$ \\
\hline 2012-2018 & $2(5 \%)$ & $9(24 \%)$ & $13(35 \%)$ & $13(35 \%)$ & $0.0241^{*}$ \\
\hline \multicolumn{6}{|c|}{ range/children } \\
\hline$<2 \mathrm{mg} / \mathrm{L}$ & $2(29 \%)$ & & $4(57 \%)$ & $1(14 \%)$ & \\
\hline $2-5 \mathrm{mg} / \mathrm{L}$ & $4(7 \%)$ & $9(17 \%)$ & $15(28 \%)$ & $26(48 \%)$ & \\
\hline $5-10 \mathrm{mg} / \mathrm{L}$ & $3(5 \%)$ & $11(18 \%)$ & $9(15 \%)$ & $39(63 \%)$ & $0.0034^{\top}$ \\
\hline $10-20 \mathrm{mg} / \mathrm{L}$ & $2(13 \%)$ & & $2(13 \%)$ & $11(73 \%)$ & \\
\hline \multicolumn{6}{|c|}{ range/all patients } \\
\hline$<2$ & $2(10 \%)$ & $4(20 \%)$ & $10(50 \%)$ & $4(20 \%)$ & \\
\hline $2-5$ & $7(6 \%)$ & $18(16 \%)$ & $33(28 \%)$ & $58(50 \%)$ & \\
\hline $5 \cdot 10$ & $5(4 \%)$ & $25(19 \%)$ & $35(27 \%)$ & $66(50 \%)$ & $0.045^{t}$ \\
\hline $10-20$ & $4(15 \%)$ & $2(7 \%)$ & $4(15 \%)$ & $17(63 \%)$ & $0.011^{\dagger}, 0.037^{t}$ \\
\hline
\end{tabular}

\section{7}

Multiple-dose clinical pharmacology of the first-in-class CXCR7 antagonist ACT-1004-1239: safety, tolerability, pharmacokinetics, pharmacodynamics, and concentration-QTe relationship in healthy subjects

Christine Huynh $^{1,2}$, Janneke M. Brussee ${ }^{1}$, Dr. Marlene Fonseca ${ }^{3}$,

Prof. Henriette E. Meyer zu Schwabedissen ${ }^{2}$, Jasper Dingemanse ${ }^{1}$, Patricia N. Sidharta ${ }^{1}$

${ }^{1}$ Idorsia Pharmaceuticals Ltd, Department of Clinical Pharmacology, Allschwil, Switzerland 
${ }^{2}$ Biopharmacy, Department of Pharmaceutical Sciences, University of Basel , Basel, Switzerland

${ }^{3}$ BlueClinical Phase 1, Hospital de Prelada, Porto, Portugal

Introduction: Antagonism of the chemokine receptor CXCR7 has shown promising effects in diverse disease areas such as oncology and immunological disorders through modulation of the systemic concentrations of its ligands, CXCL11 and CXCL12. Single-dose administration of ACT-1004-1239, a first-in-class, selective, and potent CXCR7 antagonist, revealed favorable safety, tolerability, pharmacokinetics (PK), and pharmacodynamics (PD) profiles allowing for a once-daily dosing regimen in successive clinical studies (Huynh et al., 2021)*.

Objective: This study was conducted to assess multiple-dose safety, tolerability, PK, PD, and the concentration-QT relationship of oral ACT1004-1239 in healthy male and female subjects.

Methods: This randomized, double-blind, placebo-controlled Phase 1 study enrolled a total of 30 healthy male and female subjects (sex ratio $1: 1)$ to receive 30,100 , or $200 \mathrm{mg}$ oral ACT-1004-1239 $(\mathrm{n}=24)$ or matching placebo $(n=6)$, once daily for 7 days. Safety, tolerability, PK, and PD using the plasma biomarkers of target engagement CXCL11 and CXCL12 were monitored for up to 8 days following the last dose. The relationship between ACT-1004-1239 concentrations and the QTc interval was assessed at steady-state conditions using a linear-mixed effect model based on ECG data collected during 24-h Holter monitoring. The previously established PK/PD model was updated with data obtained from this study.

Results: ACT-1004-1239 was well tolerated up to and including $200 \mathrm{mg}$ following multiple-dose administration. The adverse event (AE) incidence was similar between active treatment $(79.2 \%)$ and placebo $(83.3 \%)$. The most commonly reported AEs in $\geq 3$ subjects were headache, abnormal ECG T wave, incomplete right bundle branch block, and soft feces. No severe or serious AEs were reported, with 2 moderate AEs and the remainder of mild intensity. One female subject prematurely discontinued the study on Day 4 due to diarrhea. Based on the concentration-QTc analysis there was no association between ACT-1004-1239 plasma concentrations and QTc interval. PK profiles at steady state were comparable to the ones observed after the first dose (Day 1). ACT-1004-1239 was rapidly absorbed as indicated by tmax ranging from 1.75 to $3.01 \mathrm{~h}$. Its disposition occurred in a triphasic way with a terminal $\mathrm{t} 1 / 2$ of approximately $19 \mathrm{~h}$ across the tested dose range. ACT-1004-1239 reached steady-state conditions by Day 3 with an accumulation index of 1.2. There were no changes in CXCL11 plasma concentrations observed across the investigated dose range and when compared to placebo. CXCL12 plasma concentrations increased dose-dependently up to 2 -fold at the highest dose group (200 mg) when compared to baseline or placebo. Comparable CXCL12 exposures at the $100 \mathrm{mg}$ and $200 \mathrm{mg}$ dose levels suggest that the maximum effect of ACT-1004-1239 on the target engagement biomarker CXCL12 was achieved at the $100 \mathrm{mg}$ dose level. The updated PK/PD model (a 3-compartment PK model with non-linear distribution and an indirect-response PD model) can be utilized for predictions for future clinical studies.

Conclusion: Multiple oral doses of ACT-1004-1239, a first-in-class CXCR7 antagonist, revealed favorable safety, tolerability, PK, and PD profiles allowing for further clinical development.

*Huynh et al., Clin Pharmacol Ther. 2021 Jan 6. doi: https://doi.org/10. $1002 /$ cpt. 2154

111

Initiation of Pharmacogenetically Actionable Drugs in Discharged Hospital Patients in Finland

MSc Student Noora Kulla ${ }^{1,2}, \mathrm{MD}, \mathrm{PhD}$ Kristiina Cajanus ${ }^{1,2}$, MD, PhD Ville Kytö ${ }^{3,4}$, MD, PhD Aleksi Tornio ${ }^{1,2}$

${ }^{1}$ Integrative Physiology and Pharmacology, Institute of Biomedicine, University of Turku, Turku, Finland

${ }^{2}$ Unit of Clinical Pharmacology, Turku University Hospital, Turku, Finland
${ }^{3}$ Heart Center, Turku University Hospital and University of Turku, Turku, Finland

${ }^{4}$ Research Center of Applied and Preventive Cardiovascular Medicine, University of Turku, Turku, Finland

INTRODUCTION: Drugs associated with clinically actionable pharmacogenetic prescribing guidelines are frequently used. A potential target population to implement wide-scale pre-emptive pharmacogenetic testing is hospitalized patients who are already in contact with healthcare system and use drugs more commonly than the general population. Real-world data, however, on drug utilisation in the intended target population is needed to evaluate the impact and cost-effectiveness of pharmacogenetic testing.

OBJECTIVES: The purpose of this retrospective, register-based pharmacoepidemiologic study was to analyse post-discharge purchases of drugs with an actionable pharmacogenetic prescribing guideline in a patient cohort consisting of Finnish hospital patients.

METHODS: Adult patients treated in surgical or internal medicine specialist care wards (including subspecialties such as orthopaedics and cardiology) during 2008 to 2014 were identified from national registry data ( $n=1.42$ million; median age 59 years). Each patient was followed from the initial hospital admission for two years or until death. Altogether 33 actionable drugs associated with 10 different genes and 3 HLA-alleles were identified from prescribing guidelines compiled by the Clinical Pharmacogenetics Implementation Consortium. For each drug of interest, new drug purchases (defined as no purchases of the drug during the preceding year of hospitalisation) during the two-year follow-up were identified to calculate the incidence of drug use. Similar analyses were performed for each gene of interest. Impact in Finnish population was studied by combining the drug incidence data with Finnish phenotype frequencies reported in the literature. Differences in post-discharge drug purchases between the surgical and internal medicine wards were studied with Cox proportional-hazards model.

RESULTS: During two-year follow-up of discharged patients, $60 \%$ of the patients purchased at least one of the drugs of interest, and drug purchases of $22 \%$ of the patients were associated to $\geq 2$ different genes. Most frequently initiated drugs during two-year follow-up were ibuprofen $(25.0 \%)$, codeine $(19.4 \%)$, pantoprazole $(12.5 \%)$, simvastatin $(5.9 \%)$ and warfarin $(5.4 \%)$. The surgical unit patients had a greater probability to overall drug purchases (Hazard ratio [HR] $1.23,95 \%$ confidence interval [CI] 1.22-1.23) and to purchase drugs associated with e.g. CYP2C9 (HR 1.66, CI 1.651.67) and CYP2D6 (HR 1.54, CI 1.53-1.55) whereas patients treated in internal medicine ward were more likely to purchase drugs associated with SLCO1B1 (HR 0.41, CI 0.41-0.42), CYP2C19 (HR 0.64, CI 0.63-0.64) and VKORC1 (HR 0.28, CI 0.28-0.28).

CONCLUSION: Patients treated in surgical and internal medicine wards frequently initiate drugs associated with pharmacogenetic prescribing guidelines in the outpatient setting after being discharged from the hospital. Frequent use of codeine and non-steroidal anti-inflammatory drugs explained the more frequent overall use of drugs in the surgical cohort, and overall the results are in line with the expected profile of drug use in surgical and internal medicine patients. The results of the current study can be utilised in targeting pre-emptive pharmacogenetic testing and to appraise its cost-effectiveness.

112

Ibrutinib exposure is greatly increased by posaconazole

Mr Aleksi Olkkola ${ }^{\mathbf{1}, \mathbf{3}}$, Mr Janne Backman ${ }^{1,3}$, Mr Aleksi Tornio $^{2}$, Mrs Milka Hauta-aho ${ }^{1,3}$, Mrs Outi Lapatto-Reiniluoto ${ }^{1,3}$,

Mrs Tuija Tapaninen ${ }^{1,3}$, Mr Mikko Niemi ${ }^{1,3}$, Mr Mikko Neuvonen ${ }^{1,3}$, Mr Pertti J. Neuvonen ${ }^{1,3}$

${ }^{1}$ Department of Clinical Pharmacology, University of Helsinki and Helsinki University Hospital, Helsinki, Finland 
${ }^{2}$ Institute of Biomedicine, Faculty of Medicine, University of Turku, Turku, Finland

${ }^{3}$ Individualized Drug Therapy Research Program, Helsinki, Finland

Introduction: Ibrutinib is a Bruton's tyrosine kinase inhibitor, which has been shown to be effective in the treatment of chronic lymphocytic leukemia, as well as other B-cell malignancies. Despite its efficacy, patients treated with ibrutinib are susceptible to infections, including invasive fungal infections. Due to ibrutinib's extensive first-pass metabolism via cytochrome P450 (CYP) 3A4 in the intestine and the liver, its concentrations are greatly increased when used concomitantly with strong CYP3A4 inhibitors. Posaconazole is used in prevention and treatment of invasive fungal infections. It is a strong CYP3A4 inhibitor, but its effect on ibrutinib exposure has not been studied.

Objectives: The aim of the study was to characterize the effect of posaconazole on the pharmacokinetics of ibrutinib in healthy volunteers and to evaluate whether the drug-drug interaction could be alleviated with staggered dosing of ibrutinib and posaconazole.

Methods: The study was a randomized, placebo-controlled, 3-phase crossover study for which eleven healthy adult male volunteers were recruited. In phases 1 and 2, study participants ingested $300 \mathrm{mg}$ posaconazole twice daily for one day, followed by $400 \mathrm{mg}$ once daily thereafter either at $8 \mathrm{AM}$ (phase 1) or $9 \mathrm{PM}$ (phase 2) as a pretreatment. In phase 3, placebo was administered as a pretreatment. In the morning of the study day, at 1 hour (phase 1) or 12 hours (phase 2) after the previous dose of posaconazole or 1 hour after placebo (phase 3), a single dose of ibrutinib was admistered. For safety reasons, ibrutinib dose was $30 \mathrm{mg}$ in phase $1,70 \mathrm{mg}$ in phase 2 and $140 \mathrm{mg}$ in phase 3 . Blood samples were collected at regular intervals to determine drug concentrations. Ibrutinib concentrations were dose-adjusted to a $140-\mathrm{mg}$ dose since the pharmacokinetics of ibrutinib is known to be linear.

Results: In phase 1, posaconazole increased the dose-adjusted geometric mean area under the concentration-time curve from zero to infinity (AUC0- $\infty$ ) and peak plasma concentration (Cmax) of ibrutinib 9.5-fold (90\% confidence interval (CI) $6.3-14.3 ; \mathrm{P}<0.001)$ and 8.5 -fold $(90 \% \mathrm{CI}$ 5.7-12.8; $\mathrm{P}<0.001)$, respectively. In phase 2 posaconazole increased the dose-adjusted geometric mean AUC0- $\infty$ and Cmax of ibrutinib 10.3-fold (90\% CI 6.7-16.0; P < 0.001) and 8.2-fold (90\% CI 5.2-13.2; P < 0.001), respectively. Posaconazole had no significant effect of the half-life of ibrutinib. No significant differences were found between the two posaconazole phases in any of the pharmacokinetic variables of ibrutinib. Conclusion: Posaconazole increased ibrutinib exposure by about 10 -fold by inhibiting its CYP3A4 mediated first-pass metabolism. As patients treated with ibrutinib are susceptible to its adverse effects, great caution should be taken when it is used concomitantly with strong CYP3A4 inhibitors. The inhibitory effect of posaconazole on the CYP3A4 mediated first-pass metabolism of ibrutinib cannot be avoided by dosing the drugs 12 hours apart.

113

Early access to medicines in special situations: use of multiple criteria decision analysis as decision tool

Mrs. Montse Gasol ${ }^{1}$, Mrs. Noelia Paco ${ }^{2}$, Mrs Laura Guarga ${ }^{2}$, Mr Josep Angel Bosch ${ }^{3}$, Mrs. Caridad Pontes ${ }^{1}$, Mrs. Mercè Obach ${ }^{2}$

${ }^{1}$ Department of Pharmacology, Therapeutics And Toxicology. Universitat Autònoma De Barcelona, Barcelona, Spain

${ }^{2}$ Servei Català de la Salut, Barcelona, Spain

${ }^{3}$ Department of Internal Medicine. Universitat Autònoma de Barcelona, Barcelona, Spain

Introduction: Early access to medicines allow the prescription of a treatment before it is available in the public formulary. Early access should only be used in isolated cases of severe, progressive conditions with lack of alternatives.
In Spain, access to imported and pre-reimbursed medicines represents a situation of special complexity since their use and financing is not clearly defined. Decision-making is difficult, and a systematic assessment procedure may help to define the exceptionality of their use and guarantee the equity of access. A Multiple Criteria Decision Analysis (MCDA) could be useful to assess and prioritize treatment requests.

Objectives: To establish a standardised procedure for the assessment and prioritization of requests of access to imported and pre-reimbursed medicines by the Catalan Healthcare System.

Methods: Three workshops were conducted by the multidisciplinary Catalan Advisory Committee on Medication in Special Situations (CAMSE, for its acronym in Catalan) to create a MCDA framework based in EVIDEM methodology.

The criteria were agreed and their relative importance was weighted using a 5-point ordinal scale with a non-hierarchical technique. A positive, negative or neutral impact was defined for qualitative criteria.

A case study was developed to test the framework and its evidence matrix was tested twice.

The framework was defined considering requirements of completeness, non-redundancy and non-overlap. The criteria were redefined to fulfil requirements and improve interpretation.

Results: A total of 12 criteria were identified and selected: 8 quantitative (severity of disease, urgency, efficacy, safety, internal and external validity, therapeutic benefit and plausibility) and 4 qualitative (therapeutic alternative, existence of precedents, management impact and costs). Quantitative criteria were scored 0 to 5, except for 'plausibility' which was dichotomous.

The relative importance of quantitative criteria was rated, with mean weights from 4.7 to 3.6 showing its relevance in the decisions. Member's profiles differed only for external validity and safety.

Conclusion: MCDA may help to structure discussions and shared decisions for heterogeneous treatment requests within a multidisciplinary board, thus improving predictability and robustness in decisions involving sensitive and complex situations.

Acknowledgments: CAMSE

\section{4}

Leave no stone unturned: current state of research in pharmacological interventions in Alzheimer's patients

Mr. Frederik Laier ${ }^{1}$, Ms. Jennifer Messner ${ }^{1}$, Mr. Benedikt Johannes Wagner ${ }^{1}$, Dr. Erika Ban ${ }^{1}$

${ }^{1}$ UMFST, Tirgu Mures, Romania

Introduction: Diseases that lead to an impairment of the central nervous system often result in a massive impairment of the patient's cognitive and motor skills as well as an enormous restriction of everyday life. The diagnosis of Alzheimer's disease thus always represents a life-changing event. The disease is still considered incurable despite intensive research efforts. So far, there is no therapy for Alzheimer's disease patients that can stop the progression of the disease or lead to a complete cure. The available therapeutic approaches thus pursue solely the treatment of Alzheimer's-associated symptoms.

Objectives: The objective of the present paper was to evaluate quality of life and therapy used among Alzheimer's disease patients during an ad hoc meeting and to make correlation to the new possible therapeutic approaches in this devastating illness.

Methods: We have performed an observational, non-interventional personal interview-based evaluation during a flashmob involving Alzheimer's disease patients, collecting data from the 19 participants and their relatives. Based on data collected we made correlation with the newest therapeutic possibilities, their effect on reduction of the most complained symptoms according to the currently ongoing research and the therapeutic guidelines.

Results: We have questioned a total of 19 patients with the diagnosis of Alzheimer's disease and relatives in 14 cases. Evaluation returned that the most frequent therapy used was Memantine and $16 \%$ of the patients 
received triple therapy with: Memantine, Donepezil and Aripiprazole. $63.13 \%$ stated that they have a quality of life lower than 3 in a score ranging from 1 to 5 . According to the newest research the National Institutes of Health's clinicaltrials.gov database currently lists 582 active clinical trials dealing with $\mathrm{AD}$. 450 of these trials are investigating the efficacy of pharmacological or biological interventions and adjuvant therapies. These include drugs that not only treat the symptoms of AD but could also have an impact on disease progression. According to the S3 guideline Dementias of the German Society for Psychiatry and Psychotherapy, Psychosomatics and Neurology (DGPPN) and the German Society for Neurology (DGN), acetylcholinesterase inhibitors and the non-competitive NMDA antagonist memantine are recommended for pharmacological therapy.

Conclusion: Alzheimer's disease affects around 10 million persons each year and during COVID-19 pandemic this number increased with $16 \%$ in the US and circa $10-20 \%$ worldwide. It's been more than 17 years since the FDA last approved an Alzheimer's drug. The newest approval is due in the second trimester current year for aducanumab. With our correlational study we would like to underline the necessity of a very individualized therapeutic protocol and caregiving for dementia patients.

115

\section{Risk factors for QT prolongation among SARS-CoV2 positive} patients treated with hydroxichloroquine MD, PhD Ana Aldea-Perona a ${ }^{1,2}$, Pharm, PhD. Elena González Colominas ${ }^{3}$, Md, PhD Julián Mateus Rodríguez ${ }^{1,2}$, MD Patricia Díaz Pellicer ${ }^{1,2}$, Pharm Marta De Antonio Cuscó ${ }^{3}$, Pharm Jaime Barcelo Vidal ${ }^{3}$, Pharm Xenia Fernandez sala ${ }^{3}$, Mrs Iris Lioba Matilla Vaz ${ }^{1}$, Mrs Marta Perez Otero ${ }^{1}, \mathrm{PhD}$ Claudia Prats Balado ${ }^{1}$, Pharm, PhD Olivia Ferrandez Quirante $^{3}$, Pharm, PhD Santiago Grau Cerrato ${ }^{3}$

${ }^{1}$ Clinical Research Unit. Hospital Del Mar Medical Research Institute (IMIM), Barcelona, Spain

${ }^{2}$ Clinical Pharmacology Section. Hospital del Mar. PSMAR, Barcelona, Spain

${ }^{3}$ Pharmacy Service. Hospital del Mar. PSMAR., Barcelona, Spain

Introduction: Patients who received Hydroxychloroquine (HQ) for COVID19 showed a high risk of QTc prolongation in observational series ${ }^{1}$ Objectives: To examine EKG abnormalities in patients treated with $\mathrm{HQ}$ and to evaluate the risk factors for QT prolongation.

Methods: A retrospective observational study EUPAS35528. Main inclusion criteria: patients admitted at Hospital del Mar, Barcelona, between 15th of march and 9th of April of 2020 with positive RT-PCR test, HQ treated and EKG monitoring. QTc value was automatically calculated by the EKG device. Tisdale Risk Score for QT prolongation ${ }^{2}$ was used.

Results: 386 of 882 patients were included (496 without PCR test or EKG pre or post HQ), 58\% male. The mean of age was 64 (SD 17), $51 \%$ over than $65 \mathrm{y} .24 \%$ had some cardiovascular disease, $8.3 \%$ Thyroids' disorder, $7.3 \%$ kidney disease, $4.4 \%$ cancer. 25 (6.5\%) left ventricular hypertrophy, 19 (5\%) bradycardia and 11 (3\%) QTc segment greater than $500 \mathrm{~mm}$ were detected in baseline EKG. The median number of drugs on admission was 7 (IQR 2-12) but only 28 (7\%) used drugs with a defined risk of QT prolongation in admission. The median starting HQ dose was $800 \mathrm{mg}$ (IQR 400-800). 45\% received a cumulative dose of $2500 \mathrm{mg}$ (ranged from 800 to $5000 \mathrm{mg}$ ) with a mean duration of 6 days. 363 (94\%) patients used azithromycin too. Regarding baseline plasma K, $\mathrm{Mg}$ and $\mathrm{Ca}$ values, the means were 3.57 (SD 1.35), 1.95 (SD 1.2) and 7.5 (SD 1.95), respectively. But, 65 patients had hypokalaemia and 23 hypomagnesaemia before starting HQ. There were no statistically significant differences in these parameters $(p=0.24)$ between the patients who presented QTc greater or less than $500 \mathrm{~mm}$ at day 3 of treatment. None of the 25 patients with QTc prolongation at day 3, had a high Tisdale Risk Score. Only 3 had a moderate risk score. 15 patients with QTc below $500 \mathrm{~mm}$ increased above 500 after 3 days of treatment, 12 out of a total of 111 patients at 7 days. There were no statistically significant differences in first dose of HQ $(\mathrm{p}=0.5)$, total cumulative dose $(\mathrm{p}=0.3)$, age $(\mathrm{p}=$ $0.05)$ and about concomitant medications in admission $(\mathrm{p}=0.1)$.

Conclusion: Patients who received HQ for first wave of COVID19 were more frequently elderly and polymedicated. The proportion of patients with QT segment above $500 \mathrm{~mm}$ was lower than other published series and had a low or moderate previous risk score.

References:

1. JAMA Netw Open. 2021 Apr1;4(4):e216842

2.Circ Cardiovasc Qual Outcomes. 2013 Jul;6(4):479-87

\section{6}

Cobicistat Increases Simvastatin Exposure Over 60-fold by Inhibiting CYP3A4 and OATP1B1 in the Liver and in the Intestines

Taavi Kaartinen $^{1,2}$, Aleksi Tornio ${ }^{3,4}$, Outi Lapatto-Reiniluoto ${ }^{1,2}$, Mikko Niemi $^{1,2}$, Janne Backman ${ }^{1,2}$

${ }^{1}$ Department of Clinical Pharmacology, Faculty of Medicine, University of Helsinki and HUS Helsinki University Hospital, Helsinki, Finland

${ }^{2}$ Individualized Drug Therapy Research Program, Faculty of Medicine, University of Helsinki, Helsinki, Finland

${ }^{3}$ Integrative Physiology and Pharmacology, Institute of Biomedicine, University of Turku, Turku, Finland

${ }^{4}$ Unit of Clinical Pharmacology, Turku University Hospital, Turku, Finland

INTRODUCTION: Cobicistat, a potent mechanism-based inhibitor or cytochrome P450 (CYP) 3A4 is used as pharmacokinetic enhancer of HIV-1 protease inhibitors. It is structurally related to ritonavir and has previously increased the area under plasma concentration-time curve (AUC) of orally administered midazolam by over 19 -fold.

OBJECTIVES: The objective of this study was to characterize the inhibitory effect of cobicistat on the enzymatic activity of intestinal and hepatic CYP3A4 in healthy volunteers after pretreatment with a typical clinically used daily dosing of cobicistat by using orally administered simvastatin and intravenously administered midazolam as index substrate drugs to intestinal and hepatic CYP3A4 activity, respectively. An additional objective of the study was to estimate the time-course of recovery of enzymatic activity of CYP3A4 after discontinuation of cobicistat treatment using the observed pharmacokinetics of simvastatin and midazolam.

METHODS: We conducted a 5-phase fixed order pharmacokinetic study, where nine healthy volunteers were administered a $0.1-\mathrm{mg}$ intravenous dose of midazolam and a 20 -mg oral dose of simvastatin 1, 25, 49 and 73 hours after pretreatment with $150 \mathrm{mg}$ of cobicistat once daily for 3 or 4 days. Blood samples were collected up to 23 hours in each phase. Plasma concentrations and pharmacokinetic parameters of the above drugs and their metabolites were determined. A regression model was used to estimate the turnover half-life of CYP3A4 based on the pharmacokinetic parameters of simvastatin and midazolam.

RESULTS: The AUC of simvastatin was increased 62.0 -fold (90\% confidence interval (CI) 30.7-125.4) and 26.7-fold (90\% CI 14.7-59.2) in the 1-hour and 25-hour phases, respectively, and no statistically significant increase in the AUC was observed in the 49-hour or 73-hour phases. The clearance of midazolam was decreased to $18 \%$ (90\% CI $13-24 \%$ ), $37 \%$ (90\% CI 26-47\%), and 67\% (53-84\%) in the 1-hour, 25-hour and 49-hour phases, respectively. The turnover half-life of hepatic CYP3A4 was estimated to be 20 hours based on the recovery of clearance of intravenously administered midazolam and 30 hours for combined half-life of hepatic and intestinal CYP3A4 based on the recovery of orally administered simvastatin.

CONCLUSIONS: $150 \mathrm{mg}$ cobicistat once daily causes a strong inhibition of CYP3A4 both in the intestines and in the liver leading to a hazardous interaction when used concomitantly with simvastatin, midazolam or other sensitive substrate drugs for CYP3A4. The enzymatic activity of hepatic and intestinal CYP3A4 recovers in approximately 2-3 days after cessation of cobicistat treatment. 
Author Index

A

\begin{tabular}{|c|c|c|c|}
\hline Abad-Santos, Francisco & 51,68 & Alves, Carlos & 73,74 \\
\hline Ablij, Hans C. & 47 & Andrade, Raul J & 27 \\
\hline Abouellil, Ahmed & 53 & Andrade, Raul J & 43 \\
\hline Agema, Bram & 15 & Arias-Fuente, Carmen & 1 \\
\hline Ainla, Tiia & 22 & Arnion, Hélène & 31 \\
\hline Albendín, Helena & 76 & Arshad, Usman & 72,75 \\
\hline Albuquerque, David & 45 & Atkin, Isobel & 71 \\
\hline Aldea-Perona, Ana & 115 & Aukstikalne, Lauryna & 83 \\
\hline ALDIYAROVA, Nurgul & 40 & Avendaño Sola, Cristina & 81 \\
\hline Alonso Gómez, Beatriz & $\begin{array}{r}98,79 \\
85,96\end{array}$ & Avendaño Solá, Cristina & 61 \\
\hline Alvarez-Alvarez, Ismael & 27 & $\begin{array}{l}\text { Avendaño-Solá, } \\
\text { Cristina }\end{array}$ & 58,60 \\
\hline Álvarez Montero, Miguel & 68 & & \\
\hline
\end{tabular}

\begin{tabular}{|c|c|c|c|}
\hline al Jalali, Valentin & 82 & & \\
\hline \multicolumn{4}{|l|}{ B } \\
\hline Backman, Janne & 112,116 & Bisinella, Giulia Carlotta & 104 \\
\hline Bahmany, Soma & 70 & Biswas, Supreeti & 42 \\
\hline $\begin{array}{l}\text { Bakkum, Michiel } \\
\text { Janniek }\end{array}$ & 63 & $\begin{array}{l}\text { Blanquer-Blanquer, } \\
\text { Miguel }\end{array}$ & 76 \\
\hline Balasso, Valentina & 50 & Boldueva, Svetlana & 90 \\
\hline Ban, Erika & 114,66 & Bonilla, Elvira & 27 \\
\hline Bán, Erika-Gyöngyi & 10 & Bos, Maaike & 87 \\
\hline Banerjee, Sanatan & 42 & Bosch, Josep Angel & 113 \\
\hline Barcelo Vidal, Jaime & 115 & Bosilkovska, Marija & 25 \\
\hline Bareikis, Karolis & 41 & Brassai, Attila & 66 \\
\hline Barriocanal, Ana Maria & $\begin{array}{c}46,59 \\
49\end{array}$ & Brassai, Attila & 1 \\
\hline Barrot, Claire-Cécile & 31 & Bravo-Grande, José L. & 1 \\
\hline $\begin{array}{l}\text { Batel-Marques, } \\
\text { Francisco }\end{array}$ & 73,74 & Brozmanova, Hana & 106 \\
\hline $\begin{array}{l}\text { Bautista Blazquez, } \\
\text { Andrea }\end{array}$ & 96 & Brussee, Janneke M. & 107 \\
\hline $\begin{array}{l}\text { Bautista Blázquez, } \\
\text { Andrea }\end{array}$ & $\begin{array}{l}98,79 \\
85\end{array}$ & & \\
\hline Begam, Sabnam & 35 & Burashnikova, Irina & 69 \\
\hline Berger, Benjamin & 23,24 & Burunat-Ruesgas, Laura & 13 \\
\hline Bilal, Muhammad & 53 & Büsker, Sören & 82 \\
\hline
\end{tabular}

C

\begin{tabular}{|c|c|c|}
\hline $\begin{array}{l}\text { Caballero Bermejo, } \\
\text { Antonio }\end{array}$ & $61,81,84$ & Cherkashov, Andrey 32 \\
\hline $\begin{array}{l}\text { Caballero-Bermejo, } \\
\text { Antonio }\end{array}$ & 58,60 & \\
\hline
\end{tabular}

Cajanus, Kristiina

111 Christiaens, Thierry 63
Camargo Mamani,

Paola Águeda

Campodónico,

Diana María

Cancho, Bárbara

Carrillo-Alcaraz, Andrés $\quad 76$

Casajús Rey, Ana

Cassany, Salvador

Castro-Rebollo,

Pedro

Centeno Soto,

Gustavo

Centeno Soto,

Gustavo Adolfo

Cervino-Rodriguez, Miriam
68

51,68

Cifrián Martínez,

José Manuel

Cohen, Daisy

48

Cole, Samuel

Thomas

Coleman, Jamie

Coren, Freda

51,68

28

76

61,60

81,84

Costa, João N.

Cuellar Gómez, Diana Zulema

Cuéllar Gómez, Diana Zulema

Cueto-Sanchez, Alejandro

81, $84 \quad$ Cucurull, Esther 50

13 Curtin, François 19

Cvijić, Tara
D

\begin{tabular}{|c|c|c|c|}
\hline Das, Abhijit & 42 & $\begin{array}{l}\text { Díaz Pellicer, } \\
\text { Patricia }\end{array}$ & 115 \\
\hline De Antonio Cuscó, Marta & 115 & & \\
\hline $\begin{array}{l}\text { De Cos Cossío, M } \\
\text { Angeles }\end{array}$ & 96 & $\begin{array}{l}\text { Di Trana, } \\
\text { AnnaGiulia }\end{array}$ & 55 \\
\hline De Laiglesia, Almudena & 58 & Dima, Lorena & 63 \\
\hline Déguilhem, Amélia & 100 & Dingemanse, Jasper & 107,24 \\
\hline Desmeules, Jules & 25 & Dingemanse, Jasper & 23 \\
\hline $\begin{array}{l}\text { Desmeules, Jules } \\
\text { Alexandre }\end{array}$ & 20,19 & $\begin{array}{l}\text { Dorthea Bruun, } \\
\text { Lina }\end{array}$ & 21 \\
\hline Dho-Nagy, Eszter Anna & 10 & Duthaler, Urs & 24 \\
\hline Diago Sempere, Elena & $\begin{array}{c}61,81 \\
84\end{array}$ & Dutta, Samya & 42 \\
\hline Diago Sempere, Elena & 58,60 & Daali, Youssef & $\begin{array}{l}20,25 \\
19\end{array}$ \\
\hline \multicolumn{4}{|l|}{$d$} \\
\hline $\begin{array}{l}\text { de Cos Cossío, } \\
\text { María de los Ángeles } \\
\text { de Grouw Elke }\end{array}$ & 79,85 & de la Torre, Rafael & ique \\
\hline
\end{tabular}

E

El Tabei, Lobna $\quad 82$

F

\begin{tabular}{|c|c|c|c|}
\hline Fajgenbaum, David & 4 & Firoud, Djouher & 31 \\
\hline Farré, Magí & $\begin{array}{c}46,56,59 \\
52,49\end{array}$ & Flores, Isalud & 45 \\
\hline $\begin{array}{l}\text { Farre Albaladejo, } \\
\text { Magin }\end{array}$ & 55 & Fonseca, Marlene & 107 \\
\hline \multirow[t]{2}{*}{ Fernandez Cruz, Ana } & 84 & Freier, Anne & 71 \\
\hline & & Frenzel, Tim & 65 \\
\hline
\end{tabular}




\begin{tabular}{|c|c|c|c|}
\hline $\begin{array}{l}\text { Fernández Rozas, } \\
\text { Sonia María }\end{array}$ & & Frolov, Maxim & 92 \\
\hline $\begin{array}{l}\text { Fernandez sala, } \\
\text { Xenia }\end{array}$ & 15 & Frøyshov, Sten & 21 \\
\hline $\begin{array}{l}\text { Fernandez-Lanas, } \\
\text { Tatiana } 85\end{array}$ & & $\begin{array}{l}\text { Fuentes } \\
\text { Rodríguez, } \\
\text { María Mercedes }\end{array}$ & 79 \\
\hline $\begin{array}{l}\text { Ferrandez Quirante, } \\
\text { Olivia }\end{array}$ & 15 & Fuhr, Uwe & 72,8 \\
\hline Fijn, Roel & & Fuhr, Uwe & 53 \\
\hline \multirow{2}{*}{$\begin{array}{l}\text { Filippi-Arriaga, } \\
\text { Francesca }\end{array}$} & 0 & Fuhr, Uwe & 75 \\
\hline & & Fuster, Daniel & 59 \\
\hline \multicolumn{4}{|l|}{ G } \\
\hline Galaune, Vaidotas & 83 & $\begin{array}{l}\text { Gerbutaviciene, Rima } \\
\text { Jurate }\end{array}$ & 5 \\
\hline \multirow[t]{3}{*}{ Gallagher, Helen } & 95 & Gervasini, Guillermo & 45, \\
\hline & & Gini, Rosa & 33 \\
\hline & & Glasa, Jozef & 91 \\
\hline García Saiz, Mar & 96 & Glasova, Helena & 91 \\
\hline García Saiz, María de Mar & 98 & Gloor, Yvonne & 19 \\
\hline $\begin{array}{l}\text { García Sáiz, María del } \\
\text { Mar }\end{array}$ & $\begin{array}{l}79, \\
85\end{array}$ & Gómez Fernández, Antía & 51,68 \\
\hline García-Clavel, Jose J. & 76 & González, Luz M & 45,48 \\
\hline Garcia-Cortes, Miren & 43 & $\begin{array}{l}\text { González Colominas, } \\
\text { Elena }\end{array}$ & 115 \\
\hline García-Cortés, Miren & 27 & Gonzalez-Grande, Rocio & 43 \\
\hline García-Herráiz, Angustias & 45 & Gottardi, Massimo & 55 \\
\hline García-Pino, Guadalupe & 48 & Grau Cerrato, Santiago & 115 \\
\hline García-Saiz, Mar & 13 & Grifell, Marc & 56 \\
\hline Garitaonandia, Ibon & 71 & & \\
\hline $\begin{array}{l}\text { Garrido-Gracia, Jose } \\
\text { Carlos }\end{array}$ & 13 & Grundmann, Milan & 106 \\
\hline Gasol, Montse & 113 & Guarga, Laura & $\begin{array}{r}113 \\
28\end{array}$ \\
\hline Gatina, Ramilya & 37 & Guillen, Elena & 50 \\
\hline $\begin{array}{l}\text { Gaztelumendi Martín, } \\
\text { Gorka }\end{array}$ & $\begin{array}{l}79, \\
85\end{array}$ & & \\
\hline
\end{tabular}

H

\begin{tabular}{llll}
\hline Hallek, Michael & 72 & Hladun, Olga & $59,55,52$, \\
& 70 & Hladun, Olga & 46 \\
$\begin{array}{l}\text { Hartong, Simone } \\
\text { Hauta-aho, Milka }\end{array}$ & 112 & $\begin{array}{l}\text { Hurtado-Navarro, } \\
\text { Isabel }\end{array}$ & 93 \\
Hernández, César & 28 & $\begin{array}{c}\text { Huseyn-Zada }, \\
\text { Mikayil }\end{array}$ & 75 \\
$\begin{array}{l}\text { Hernandez-Hernandez, } \\
\text { Mireia }\end{array}$ & 13 & $\begin{array}{c}\text { Huynh, Christine } \\
\text { Munh }\end{array}$ & 107 \\
\hline
\end{tabular}

I

\begin{tabular}{llll}
\hline Ikhambayeva, Ainur & 40 & Ivaniš, Anja & 30 \\
Iturbe Fernández, David & 85 & & \\
\hline
\end{tabular}

$\mathbf{J}$

\begin{tabular}{llll}
\hline Jaehde, Ulrich & 72 & Javaloyes Garachana, Laura & $61,81,84$ \\
Jaehde, Ulrich & 75 & Javaloyes-Garachana, Laura & 58,60 \\
Jakob, Carolin & 72 & Javellaud, James & 31 \\
Jang, Haerim & 38 & Jimenez, Miguel & 43 \\
Jara-Rubio, Rubén & 76 & Jouanjus, Emilie & 100,99 \\
Jardou, Manon & 31 & Jäger, Walter & 82 \\
\hline
\end{tabular}

$\mathbf{K}$

\begin{tabular}{llll}
\hline Kacirova, Ivana & 106 & & \\
Kadiseva, Elvina & 37 & Knoester, Pieter D. & 47 \\
Kalapacs, Andrea-Maria & 66 & Ko, Jae-Wook & 38 \\
Kalapács, Andrea-Mária & 10 & Koch, Birgit & 15, \\
& & & 70
\end{tabular}

Kalfsvel, Laura $\quad 44$

Kalverda-Mooij, $\quad 87 \quad$ Kooistra, Emma $\quad 65$

Kirsten

$\begin{array}{llll}\text { Kampus, Priit } & 22 & \text { Koolen, Stijn } & 15\end{array}$

Kang, MinJa $\quad 39 \quad$ Koristkova, Blanka 106

Kappers, Mariette 70

Karinauske, Egle $\quad 5,57,67 \quad$ Krähenbühl, Stephan 24

Kasciuskeviciute, $\quad 83,41,57 \quad$ Kuijvenhoven, $\quad 87$

Skaiste Marianne

Kasciuskeviciute, $67 \quad$ Kukrálová, Kateřina 103

Skaiste

Khor, Johnson $\quad 4 \quad$ Kulagina, Ludmila 37

Kim, Bongtae $\quad 38,39 \quad$ Kulagina, Lyudmila 36

Kim, Jung-Ryul $\quad 38 \quad$ Kulla, Noora $\quad 111$

Kim, Min-Gul $\quad 39 \quad$ Kytö, Ville 111

Kim, Seokuee $\quad 38 \quad$ Köllö, Zoltan 66

Klein, Gernot 23

$\mathbf{L}$

\begin{tabular}{|c|c|c|c|}
\hline & & Lenoir, Camille & $\begin{array}{l}20 \\
19\end{array}$ \\
\hline Laidmäe, Erki & 22 & Levyova, Maria & 91 \\
\hline Laier, Frederik & 114 & Likic, Robert & 63 \\
\hline Lapatto-Reiniluoto, Outi & $\begin{array}{l}112 \\
116\end{array}$ & $\begin{array}{r}\text { Lines de Cos, } \\
\mathrm{M}^{\circ} \text { Ángeles }\end{array}$ & 98 \\
\hline \multirow[t]{2}{*}{ Lapeyre-Mestre, Maryse } & 100 & $\begin{array}{l}\text { Lissenberg-Witte, } \\
\text { Birgit }\end{array}$ & 87 \\
\hline & & $\begin{array}{l}\text { Lopez Raigada, } \\
\text { Azahara }\end{array}$ & 68 \\
\hline Lasys, Tomas & 57,67 & López-Gómez, Juan & 48 \\
\hline Lavi, Tal & 97 & López-Pérez, Ricardo & 1 \\
\hline Lavin-alconero, Lucia & 13 & Lorch, Ulrike & 71 \\
\hline Lavon, Ophir & 11,97 & Lucena, M Isabel & 27 \\
\hline Lawson, Roland & 31 & Luján, María & 50 \\
\hline Leentjens, Jenneke & 65 & Lukac, Martin & 4 \\
\hline $\begin{array}{l}\text { Leguízamo-Martínez, Lina } \\
\text { M. }\end{array}$ & 50 & & \\
\hline
\end{tabular}


M

$\begin{array}{ll}\text { Maciulaitis, Romaldas } & 41 \\ \text { Mačiulaitis, Romaldas } & 63 \\ \text { Mačiulaitis } & \\ \text { Magistad K., Henrik } & 21 \\ \text { Magliocco, Gaëlle } & 25 \\ \text { Mahomedradja, } & 87 \\ \quad \text { Rashudy F } & \\ \text { Mahomedradja, } & 86 \\ \quad \text { Rashudy F. } & \end{array}$

\begin{tabular}{|c|c|c|c|}
\hline Makalkina, Larisa & 40 & Mezaache, Salim & 99 \\
\hline Maksimov, Maxim & $\begin{array}{c}36,69 \\
37\end{array}$ & & \\
\hline Mannocchi, Giulio & 55 & $\begin{array}{l}\text { Mimica Matanović, } \\
\text { Suzana }\end{array}$ & 30,14 \\
\hline Marandi, Toomas & 22 & Moon, Seol Ju & 39 \\
\hline Maříková, Martina & 103 & $\begin{array}{l}\text { Mora Cuesta, Victor } \\
\text { Manuel }\end{array}$ & 98 \\
\hline Marquet, Pierre & 31 & $\begin{array}{l}\text { Mora Cuesta, Víctor } \\
\text { Manuel }\end{array}$ & 79,85 \\
\hline Martin, Soraya & $\begin{array}{l}46,59 \\
55,49\end{array}$ & $\begin{array}{l}\text { Moraleda-Jiménez, Jose } \\
\text { M. }\end{array}$ & 76 \\
\hline Martínez, Lucía & 46 & Morris, Colette & 95 \\
\hline $\begin{array}{l}\text { Martínez-Mellado, } \\
\text { Antonio J. }\end{array}$ & 76 & Mota Zamorano, Sonia & 48 \\
\hline Martinson, Katrin & 22 & Mota-Zamorano, Sonia & 45 \\
\hline Massey, Emma & 70 & Muehlan, Clemens & 23,24 \\
\hline $\begin{array}{l}\text { Mateus Rodríguez, } \\
\text { Julián }\end{array}$ & 115 & Mukhopadhyay, Sandip & 42 \\
\hline Mathijssen, Ron & 15 & & \\
\hline Matilla Vaz, Iris Lioba & 115 & & \\
\hline Mazon Maraña, Ivan & 96 & & \\
\hline Mazón Maraña, Iván & $\begin{array}{c}98,79 \\
85\end{array}$ & & \\
\hline
\end{tabular}

$\mathbf{N}$

\begin{tabular}{|c|c|c|c|}
\hline Navarro, Belén & 58 & & \\
\hline Navarro, Mireia & 50 & $\begin{array}{l}\text { Nigmedzyanova, } \\
\text { Albina }\end{array}$ & 37 \\
\hline Navarro-Pérez, María Luisa & 48 & & \\
\hline Neuvonen, Mikko & 112 & & \\
\hline Neuvonen, Pertti J. & 112 & Niu, Hao & 27 \\
\hline $\begin{array}{l}\text { Nicolas de la Puente, Jose } \\
\text { Manuel }\end{array}$ & 13 & $\begin{array}{l}\text { Nogueiras Alvarez, } \\
\text { Rita }\end{array}$ & 96 \\
\hline $\begin{array}{l}\text { Nicolás de la Puente, José } \\
\text { Manuel }\end{array}$ & 79,85 & $\begin{array}{l}\text { Nogueiras Álvarez, } \\
\text { Rita }\end{array}$ & $\begin{array}{c}98,79 \\
85\end{array}$ \\
\hline Niederer, Amine & 20 & $\begin{array}{l}\text { Nogueiras-Alvarez, } \\
\text { Rita }\end{array}$ & 13 \\
\hline Niemi, Mikko & $\begin{array}{l}112, \\
116\end{array}$ & Nuñez, Melani & 52 \\
\hline
\end{tabular}

Méndez Rodríguez, Marta 51

Merad, Sonia 100

Messner, Jennifer $\quad 114$

Meyer zu Schwabedissen, 107 Henriette E.
O

\begin{tabular}{llll}
\hline Obach, Mercè & 113 & Oosten, Astrid & 15 \\
Ochoa Mazarro, Dolores & 51,68 & Ortega-Alonso, Aida & 43 \\
Očovská, Zuzana & 103 & & \\
Olesti, Eulàlia & 56 & Otten, René & 86 \\
Olkkola, Aleksi & 112 & & \\
\hline
\end{tabular}

Pacifici, Roberta

55

Penedones, Ana $\quad 73,74$

Paco, Noelia

113

Padervinskis,

83

Evaldas

Pajouheshnia, 33

Romin

Paolo Busardó, $\quad 55$

Francesco

Papaioannidou, 63

Paraskevi

Papaseit, Esther

46, 56, 59,

52,49

Papaseit, Esther $\quad 55$

Pareja, Ana $\quad 76$

Payares Herrera, $\quad 81,84$ Concepcion

Payares Herrera, Concepción

Payares-Herrera, 58, 60 Concepción

Pontes, Caridad $\quad 113,28$

Poschner, Stefan 82

Pecere, Alessandro 104

Poyatos, Lourdes $46,56,59,55$, 49

Peckauskas, $\quad 5$ Aurimas

Prats Balado,

115

Peeters, Laura E.J. $\quad 70$
$\mathbf{R}$

\begin{tabular}{|c|c|c|c|}
\hline & & Rodriguez-Bernal, Clara & 93 \\
\hline $\begin{array}{l}\text { Ramirez Garcia, } \\
\text { Almudena }\end{array}$ & 81,84 & $\begin{array}{l}\text { Rodríguez-Fortúnez, } \\
\text { Patricia }\end{array}$ & 76 \\
\hline $\begin{array}{l}\text { Ramirez García, } \\
\text { Almudena }\end{array}$ & 61 & $\begin{array}{l}\text { Rodríguez-Jiménez, } \\
\text { Consuelo }\end{array}$ & 76 \\
\hline $\begin{array}{l}\text { Ramirez-Garcia, } \\
\text { Almudena }\end{array}$ & 58,60 & Rogov, Vladimir & 92 \\
\hline \multirow[t]{2}{*}{ Reny, Jean-Luc } & 19 & Roig, Marta & 28 \\
\hline & & Rollason, Victoria & 20 , \\
\hline
\end{tabular}

Reumerman, Michael O. 86

Richir, Milan C.

63

Riera-Arnau, Judit

33,93

Roux, Perrine

99

Riera-Arnau, Judit 50

Roberto, Giuseppe

Ruiz Antoran, Belen

81 , 


$\begin{array}{llll}\text { Robles, Nicolás R } & 48 & \text { Ruiz-Antoran, Belén } & 60 \\ \text { Robles-Diaz, Mercedes } & 43 & \text { Ruiz-Antorán, Belén } & 61 \\ \text { Robles-Díaz, Mercedes } & 27 & \text { Ruiz-Cabello, Francisco } & 43 \\ \text { Rodríguez, Alexis } & 50 & & \end{array}$

S

\begin{tabular}{|c|c|c|c|}
\hline Sabattini, Giancarlo & 24 & & \\
\hline \multirow[t]{2}{*}{ Sadauskas, Vladas } & 57,67 & Sidharta, Patricia N. & 107 \\
\hline & & Sigaloff, Kim & 87 \\
\hline Samer, Caroline Flora & 20,19 & Sigaloff, Kim C.E. & 86 \\
\hline Sanabria-Cabrera, Judith & 27,43 & Siles, Adrián & 59 \\
\hline $\begin{array}{l}\text { Sánchez Escamilla, } \\
\text { Miriam }\end{array}$ & 79 & & \\
\hline $\begin{array}{l}\text { Sánchez Santiago, } \\
\text { Blanca }\end{array}$ & 98,96 & & \\
\hline $\begin{array}{l}\text { Sánchez Santiago, María } \\
\text { Blanca }\end{array}$ & 79,85 & & \\
\hline Sanchez-Saez, Francisco & 93 & & \\
\hline Sancho Lopez, Aranzazu & 81,84 & Solano, Elena & 76 \\
\hline Sancho López, Aranzazu & 61 & Souverein, Patrick & 33 \\
\hline Sancho-Lopez, Arantxa & 58,60 & & \\
\hline $\begin{array}{l}\text { Sanfélix-Gimeno, } \\
\text { Gabriel }\end{array}$ & 93 & Spencer, Christopher S & 71 \\
\hline Sans-Pola, Carla & 50 & Spindeldreier, Kirsten C. & 72 \\
\hline Sanz, Emilio J. & 63 & & \\
\hline Sassen, Sebastiaan & 15 & & \\
\hline \multirow[t]{2}{*}{$\begin{array}{l}\text { Sauvage, } \\
\text { François-Ludovic }\end{array}$} & 31 & Stankeviciute, Simona & $\begin{array}{c}83,57 \\
\quad 67\end{array}$ \\
\hline & & Stephens, Camilla & 43 \\
\hline Schinkel, Michiel & 86 & $\begin{array}{l}\text { Stolk-van der Heijden, } \\
\text { Charlotte }\end{array}$ & 65 \\
\hline $\begin{array}{l}\text { Seeger-Nukpezah, } \\
\text { Tamina }\end{array}$ & 72 & & \\
\hline Seo, Hyosook & 38 & & \\
\hline Shin, Naree & 39 & & \\
\hline Shteinberg, Michal & 97 & Szabados, Csongor & 10 \\
\hline
\end{tabular}

T

\begin{tabular}{llll}
\hline Tamosuitis, Tomas & 41 & Tichelaar, Jelle & $87,63,86$ \\
Tapaninen, Tuija & 112 & & \\
Taubel, Jorg & 71 & Tomàs, Mireia & 50 \\
Taubert, Max & 72,75 & Tomek, Dominik & 91 \\
Terrier, Jean & 19 & Tornio, Aleksi & $111,112,116$ \\
Thomas, Aurélien & 25 & Torrens, Marta & 56 \\
Thurin, Nicolas H & 33 & & \\
\hline
\end{tabular}

t

ter Heine, Rob $\quad 65$

$\mathbf{U}$

\begin{tabular}{llll}
\hline Ullah, Sami & $72,82,75$ & Usaite, Akvile & 67 \\
Usaite, Akvile & 57 & & \\
\hline V & & & \\
& & & \\
\hline Valencia López, David & $98,79,85,96$ & Ventura, Mireia & 56 \\
Valiullina, Inna & 36 & & \\
Van der Rijt, Carin & 15 & Versmissen, Jorie & 44,70 \\
Vare, Pille-Riin & 22 & Vila, Alexandra & 59 \\
Vega Gil, Noelia & $98,79,85,96$ & Vlček, Jiř́ & 103 \\
Vehreschild, Jörg Janne & 72 & & \\
\hline
\end{tabular}

$\mathbf{v}$

\begin{tabular}{llll}
\hline van Agtmael, Michiel & 87 & van der Net, Jeroen & 70 \\
van Agtmael, Michiel A. & 63,86 & van Dijk, Liset & 70 \\
van den Beukel, Tessa O. & 87 & van Doorn, Adriaan & 44 \\
van den Broek, Walter & 44 & van Gelder, Teun & 70 \\
van der Kuy, Hugo & 44 & van Rosse, Floor & 44 \\
\hline
\end{tabular}

W

\begin{tabular}{llll}
\hline Wagner, Benedikt Johannes & 114 & Williams, Lorna & 95 \\
Wang, Steven & 87 & Williams, Reece & 4 \\
Weig, Thomas & 75 & Witte, Anne M.C. & 47 \\
Weverling-Rynsburger, Annelies W.E. & 47 & & \\
\hline
\end{tabular}

$\mathbf{Z}$

\begin{tabular}{llll}
\hline & & Zinzi, Alessia & 109 \\
Zaliaduonyte, Diana & 5 & Zoller, Michael & 75 \\
Zander, Johannes & 75 & Zuriguel, Esperanza & 50 \\
& & Zvegintseva, Albina & $36,69,37$ \\
\hline
\end{tabular}

Publisher's note Springer Nature remains neutral with regard to jurisdictional claims in published maps and institutional affiliations. 